\title{
AERODYNAMIC ANALYSIS FOR MODULE DISCRETIZATION AND CONSOLIDATION OF A MORPHING WING
}

\author{
By \\ Ryan Perera, B.Eng \\ Aerospace Engineering \\ Ryerson University, 2011
}

\begin{abstract}
A thesis presented to Ryerson University
in partial fulfillment of the requirements for the degree of

Master of Applied Science

in the Program of

Aerospace Engineering
\end{abstract}

Toronto, Ontario, Canada, 2014

(C) Ryan Perera 


\section{Author's Declaration}

I hereby declare that I am the sole author of this thesis.

I authorize Ryerson University to lend this thesis to other institutions or individuals for the purpose of scholarly research.

I further authorize Ryerson University to reproduce this thesis by photocopying or by

other means, in total or in part, at the request of other institutions or individuals for the purpose of scholarly research. 


\section{Acknowledgements}

First and foremost I would like to acknowledge Dr. Jeff Xi and Dr. Paul Walsh for their continual support, guidance, and patience throughout my undergraduate and graduate years. I owe a lot of my success to both of them and for that I will be forever grateful.

Furthermore I would like to thank Daniel Finistauri. Your work with the modular morphing wing concept was essential to the development of this thesis. This research would not be possible if it wasn't for your ingenuity and determination to develop an exciting morphing wing concept.

I would also like to thank Szymon Buhajczuk for your guidance with the use of Ansys Workbench. Your help allowed me to not only perform accurate analysis but to also be confident in my findings. The in depth analysis would not be possible without your knowledge and amazing teaching ability.

Thank you to Prakash Paudel for playing a major role with the understanding and development of various Matlab scripts for module discretization, and geometry generation, and descent profile generation. Your contribution played an essential role in this thesis and I wish you the best of luck in your own research.

Lastly I would like to thank my family and friends for constantly providing emotional support throughout my university career. Your support has made a huge difference through the difficult times. This thesis would not be possible without you. 


\title{
Abstract
}

\section{Aerodynamic Analysis for Module Discretization and Consolidation of a Morphing Wing \\ Ryan Perera}

\author{
A thesis for the degree of Master of Applied Science, 2014 \\ Department of Aerospace Engineering \\ Ryerson University
}

\begin{abstract}
Module discretization and consolidation was performed on morphing wing profiles optimized for climb, cruise, and descent flight regimes. Wing profiles were created using an optimization algorithm based on their aerodynamic performance for the three flight regimes. A module discretization method was applied for the three cases and the minimum number of modules were found for each case without significantly sacrificing performance. The three wing profiles were then consolidated into a single final wing using a newly proposed method for combining closely aligned joints based on a weighting scale for each flight regime. When the final wing's performance was compared to the original wing profiles a reduction of 5\% and $2 \%$ was observed for climb and descent configurations, respectively. The cruise configuration was found have a $3 \%$ increase when compare to the original profile. The final wing was found to successfully maintain aerodynamic performance during module discretization and consolidation process.
\end{abstract}




\section{Table of Contents}

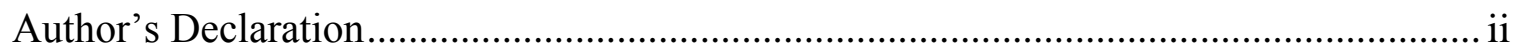

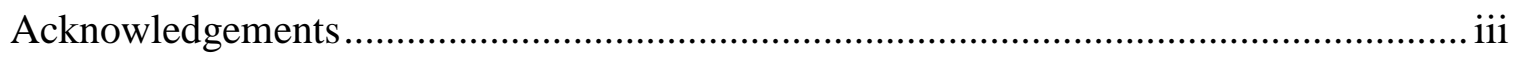

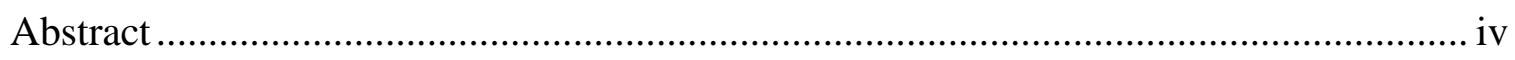

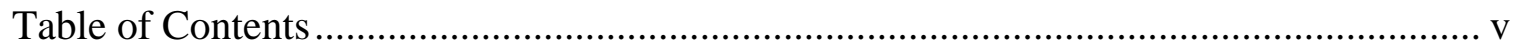

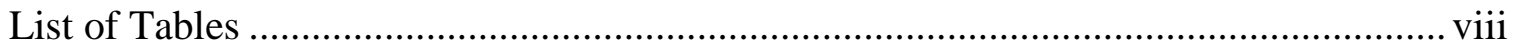

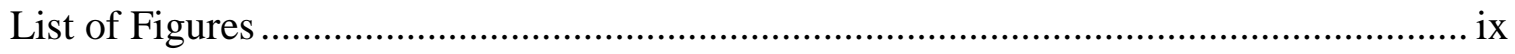

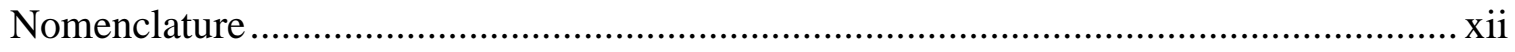

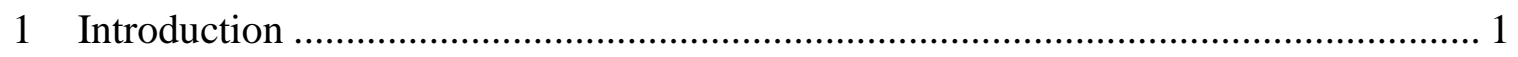

1.1 Aircraft Morphing Design ........................................................................ 2

1.2 Problem Statement .................................................................................. 4

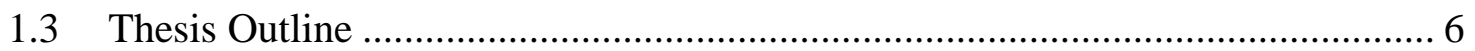

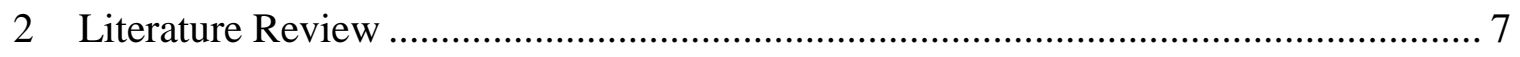

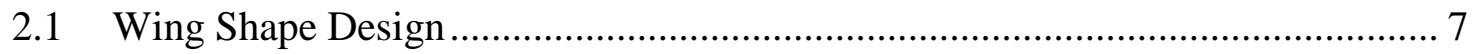

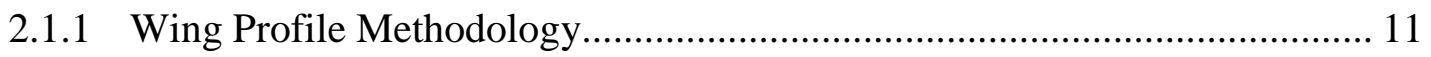

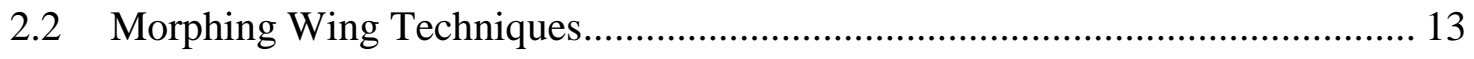




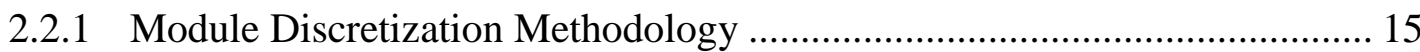

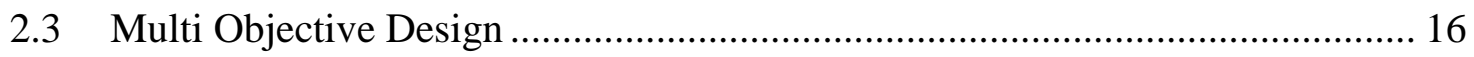

2.3.1 Wing Consolidation Methodology ……………………………………....... 18

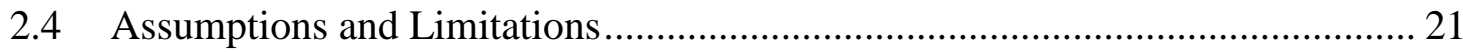

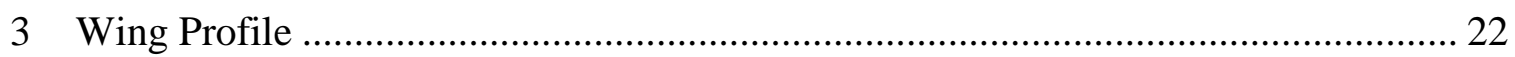

3.1 Computational Methods and Conditions ………………………................... 22

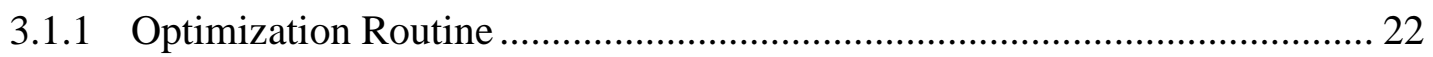

3.1.2 Computational Fluid Solver ……………………………....................... 23

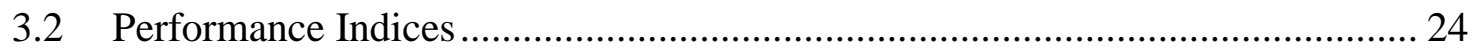

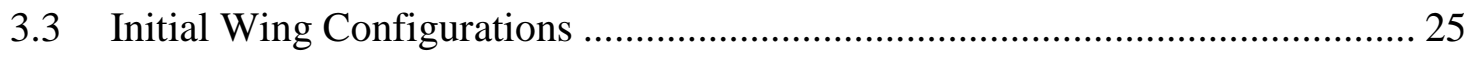

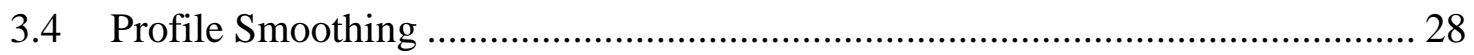

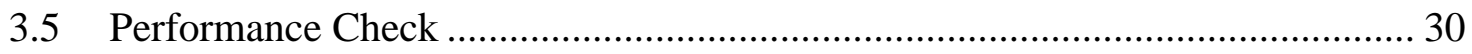

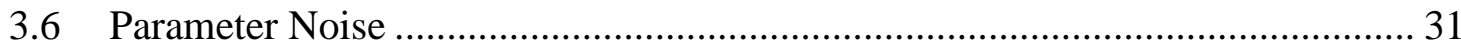

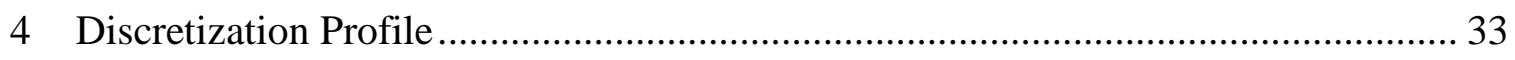

4.1 Computational Methods and Conditions ……………………………………..... 34

4.1.1 Computational Fluid Solver …………………………………………..... 34

4.1.2 Computational Grid........................................................................... 35

4.2 Discretization Methodology ........................................................................... 38

4.3 Discretize Wing Profile ................................................................................ 40

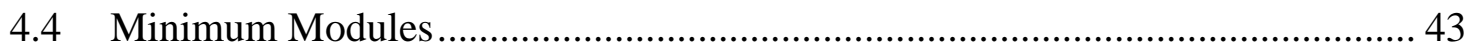

4.4.1 Climb Module Analysis .............................................................................. 43

4.4.2 Cruise Module Analysis .............................................................................. 44

4.4.3 Descent Module Analysis............................................................................. 45

4.5 Wing Performance Discussion ....................................................................... 46

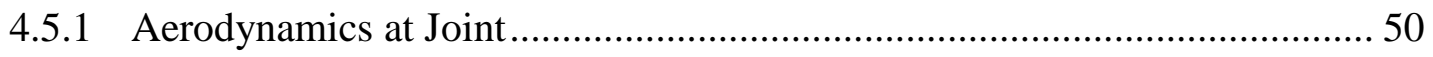




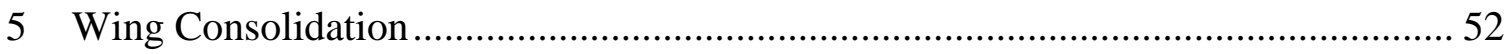

5.1 Discretized Profile Summation ..................................................................... 53

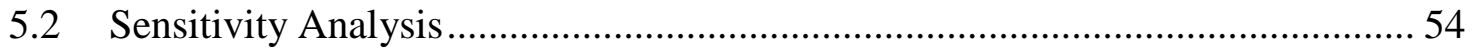

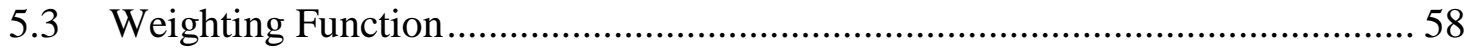

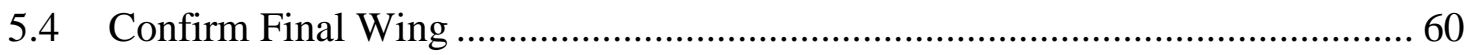

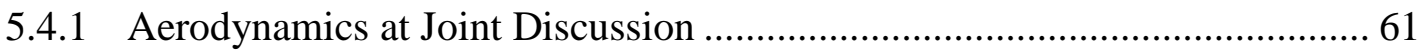

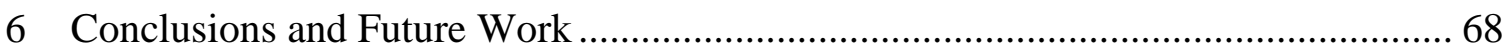

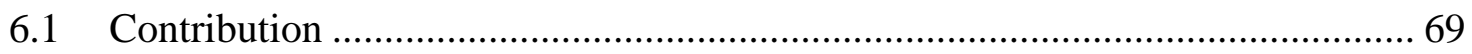

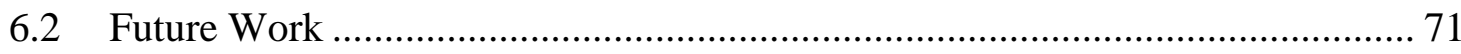

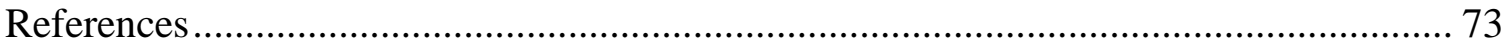

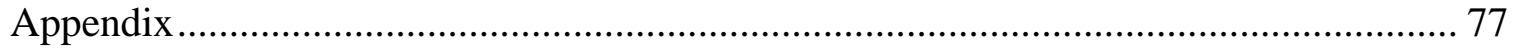




\section{List of Tables}

Table 1.1: Aircraft Morphing Historical Perspective [1,2] ........................................... 2

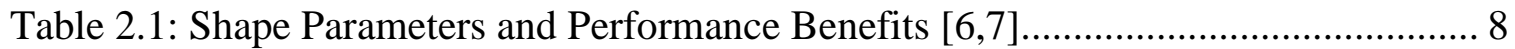

Table 3.1: Harmony Search Algorithm Parameter Limits ........................................... 23

Table 3.2: Performance Indices at Three Flight Regimes............................................. 25

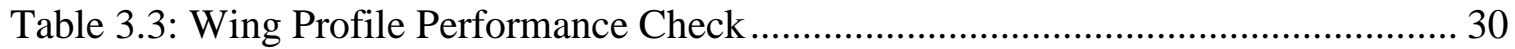

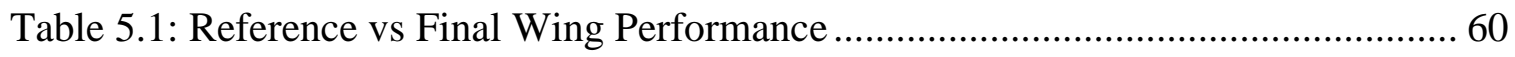

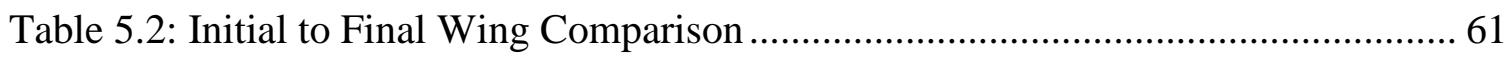

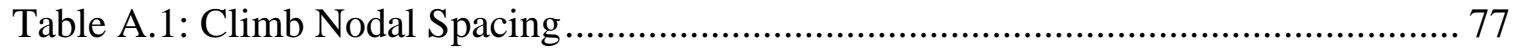

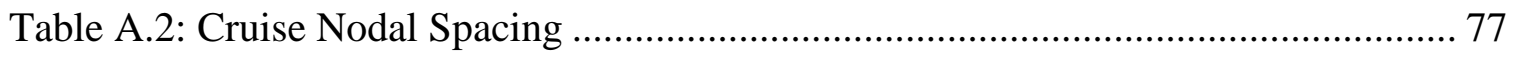

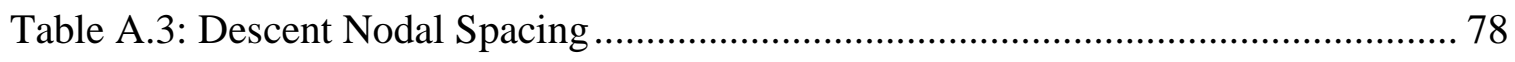

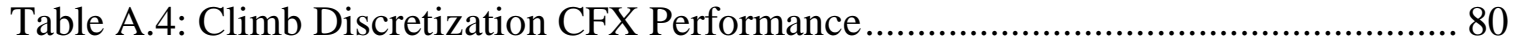

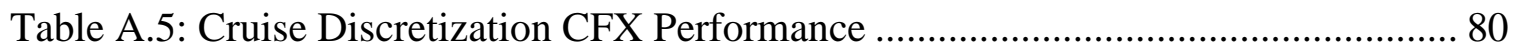

Table A.6: Descent Discretization CFX Performance ................................................. 80 


\section{List of Figures}

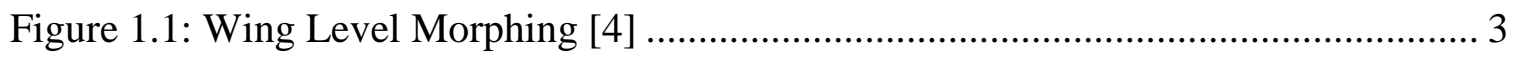

Figure 1.2: Coordinate Frame [5] ......................................................................... 3

Figure 1.3: Initial to Final Wing Strategy ……………............................................ 4

Figure 2.1: Bell X-5 Swept Arrangement [11] ......................................................... 9

Figure 2.2: Variable Gull-Wing Morphing Aircraft [12]................................................ 10

Figure 2.3: NextGen Morphing Wing Concept [2]....................................................... 11

Figure 2.4: Example A Configuration …………………….................................... 12

Figure 2.5: Example B Configuration................................................................... 12

Figure 2.6: Lockheed Martin Z-Wing [17] ................................................................ 14

Figure 2.7: Discretized Wing Tip Optimization [16]................................................... 15

Figure 2.8: Example A Discretization ..................................................................... 16

Figure 2.9: Example B Discretization..................................................................... 16

Figure 2.10: Performance of Firebee Baseline vs Morphing Wings [20] ......................... 17

Figure 2.11: Example A \& B Consolidation................................................................ 19

Figure 2.12: Final Wing Example.............................................................................. 20

Figure 3.1: Climb Initial Configuration .................................................................. 26

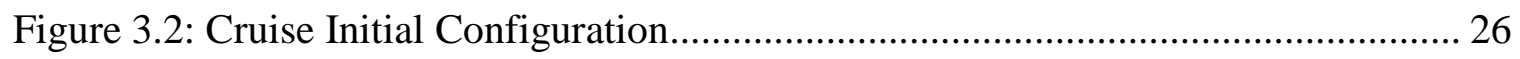

Figure 3.3: Descent Initial Configuration ………………….................................. 27

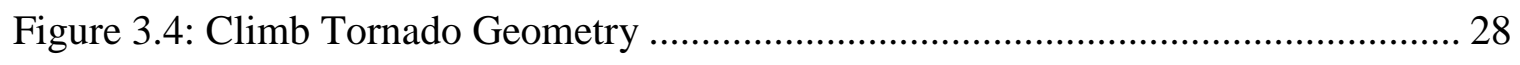

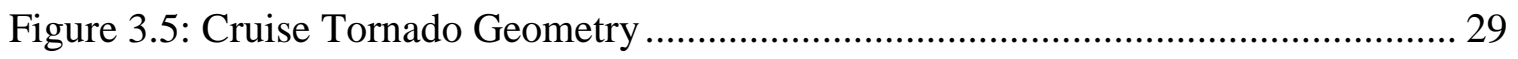




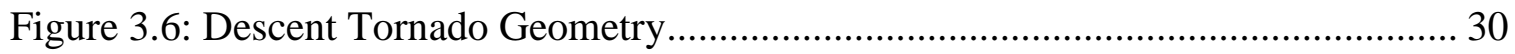

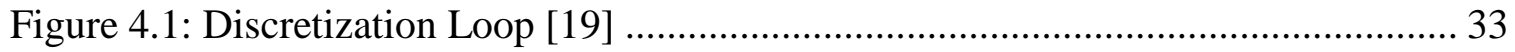

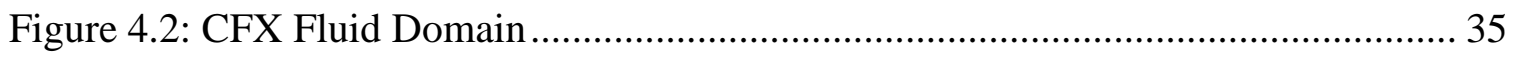

Figure 4.3: Ansys Grid Fluid Region......................................................................... 36

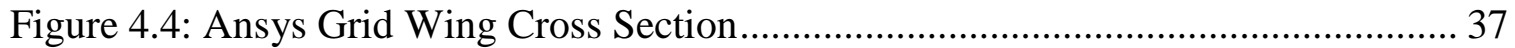

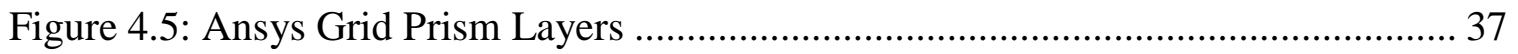

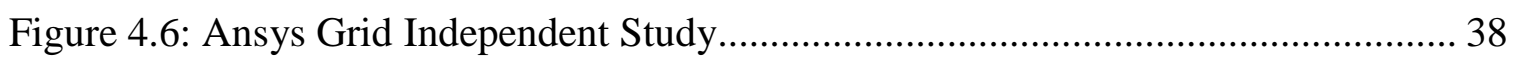

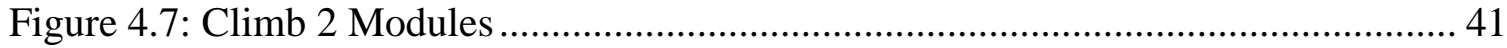

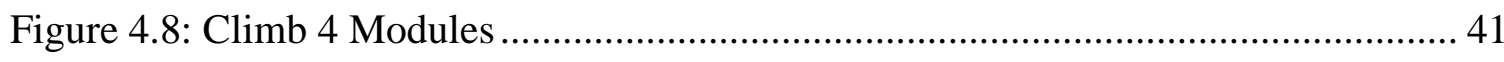

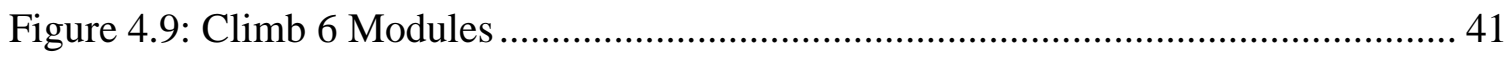

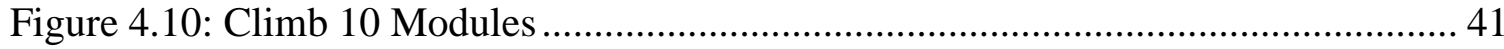

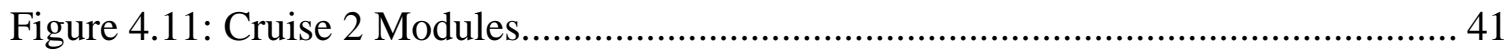

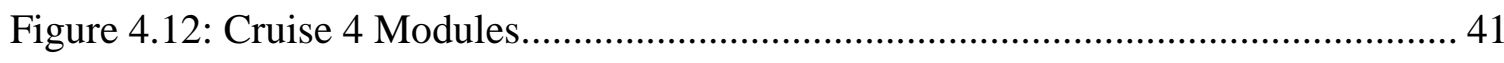

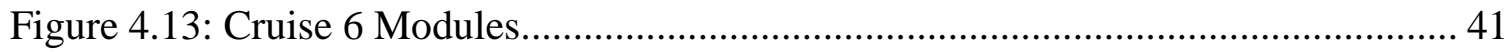

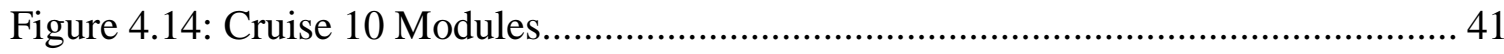

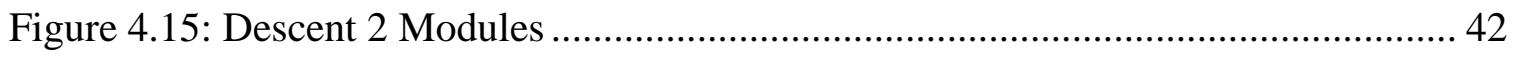

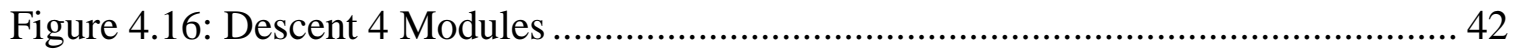

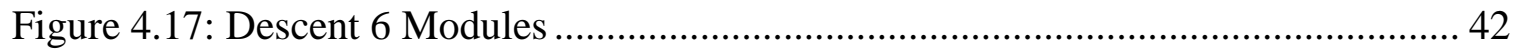

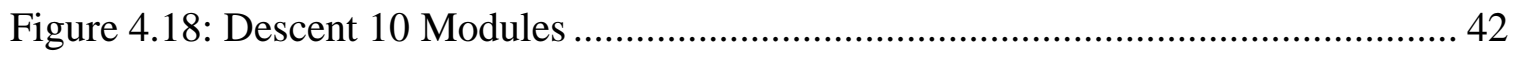

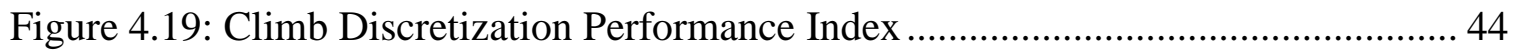

Figure 4.20: Cruise Discretization Performance Index........................................... 45

Figure 4.21: Descent Discretization Performance Index .............................................. 46

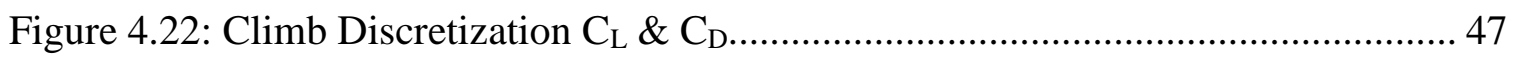

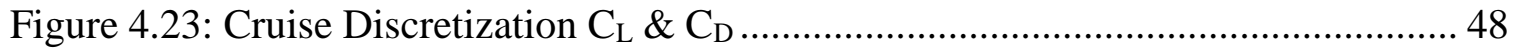

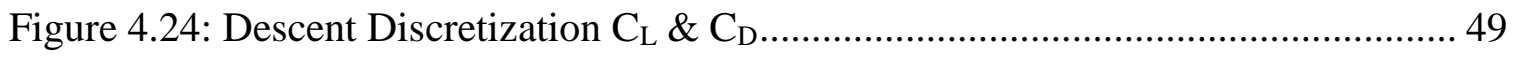

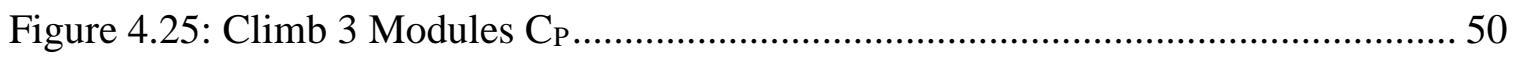

Figure 4.26: Climb 3 Module Wall Shear Stress ....................................................... 51

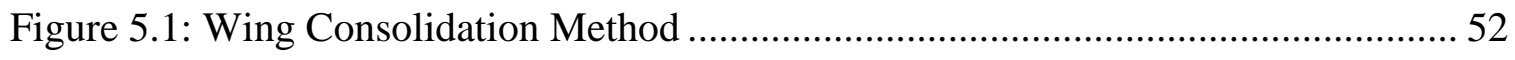

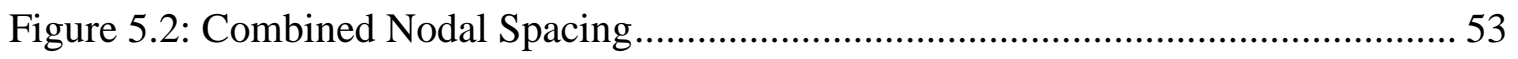

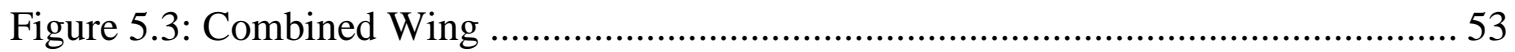

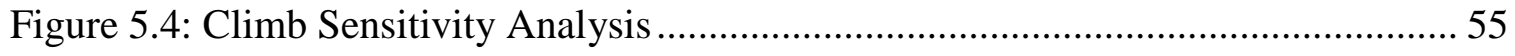




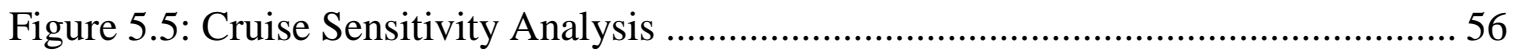

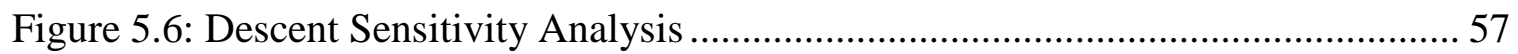

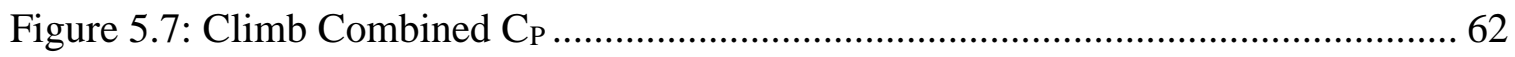

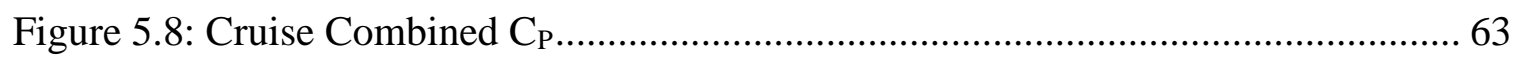

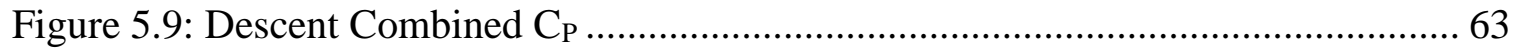

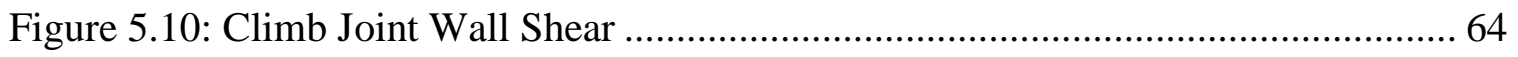

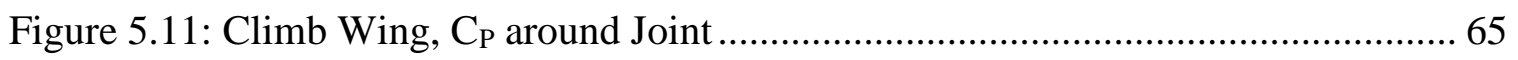

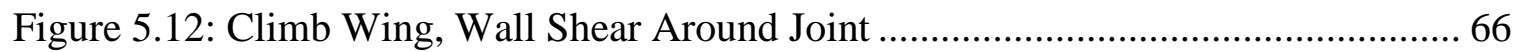

Figure A.1: Climb Smooth Profile Delta CP .......................................................... 78

Figure A.2: Cruise Smooth Profile Delta CP .......................................................... 79

Figure A.3: Descent Smooth Profile Delta CP .......................................................... 79 


\section{Nomenclature}

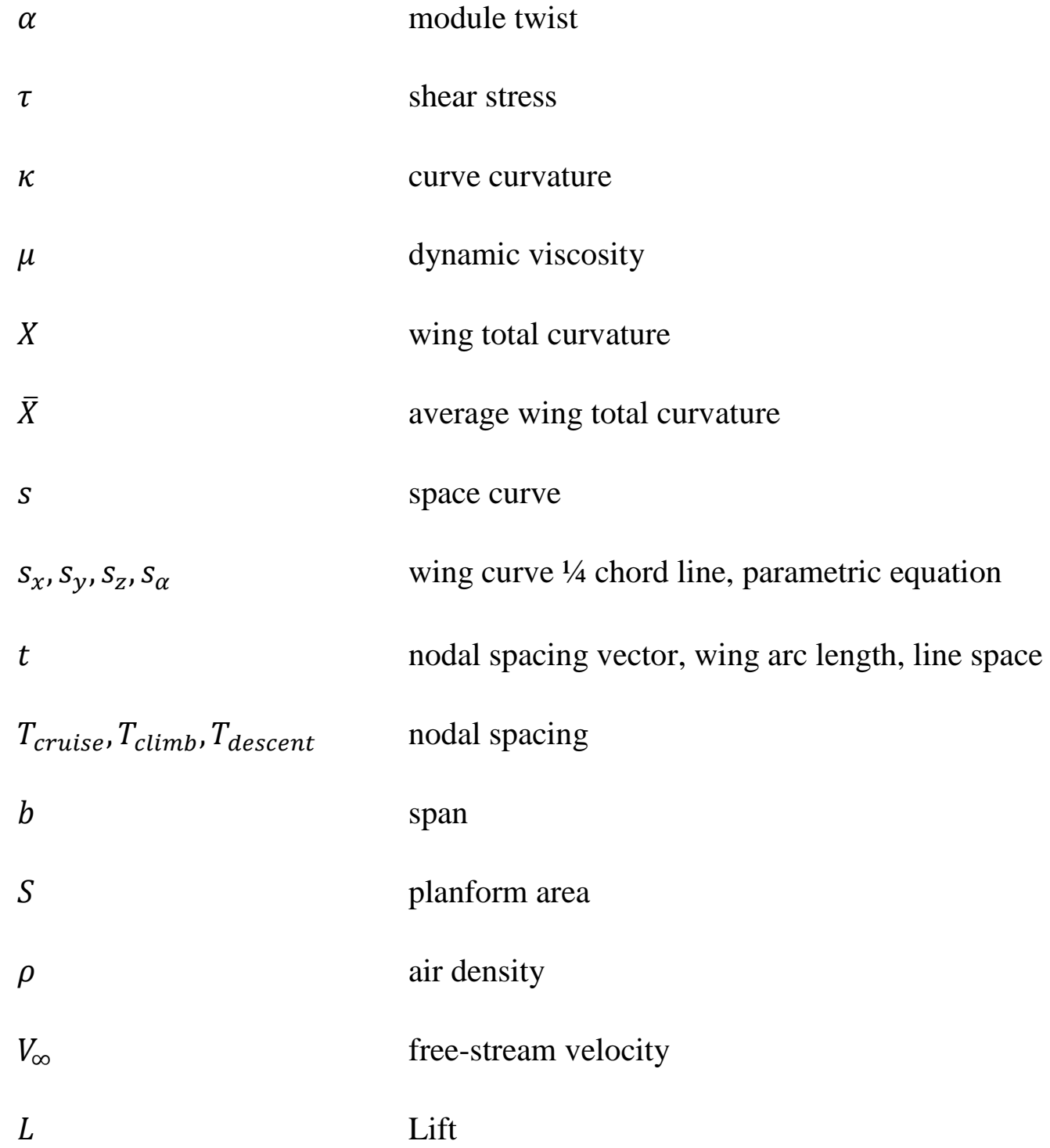

$\alpha$

$\tau$

$\kappa$

$\mu$

$X$

$\bar{X}$

$S$

$s_{x}, s_{y}, s_{z}, s_{\alpha}$

$t$

$T_{\text {cruise }}, T_{\text {climb }}, T_{\text {descent }}$

$b$

$S$

$\rho$

$V_{\infty}$

$L$

module twist

shear stress

curve curvature

dynamic viscosity

wing total curvature

average wing total curvature

space curve

wing curve $1 / 4$ chord line, parametric equation

nodal spacing vector, wing arc length, line space

nodal spacing

span

planform area

air density

free-stream velocity

Lift 


$\begin{array}{ll}D & \text { Drag } \\ c_{t} & \text { Thrust specific fuel consumption } \\ C_{L} & \text { coefficient of lift } \\ C_{D} & \text { coefficient of drag } \\ C_{D, 0} & \text { zero-lift coefficient } \\ C_{P} & \text { coefficient of pressure } \\ T & \text { Thrust } \\ W & \text { Weight } \\ R / C & \text { rate of climb } \\ R & \text { range } \\ V_{V} & \text { sink rate } \\ w f & \text { weighting function } \\ f & \text { relative performance } \\ & \end{array}$

AoA

angle of attack

DOF

degree of freedom

UAV

unmanned aerial vehicle

CFD

computational fluid dynamics 


\section{Introduction}

Ever since the Wright brothers achieved the world's first powered, heavier than air, controlled flight, people have been searching for new ways to improve the airplane. As like many engineering projects, compromises were made based on the present technology. The need to eliminate these compromises lead to break through inventions that revolutionize the industry. One of these was the development of the jet engine. An aircraft's wings have also drastically changed within the last 100 years, new materials and structures help improve strength and durability while minimizing the overall weight. Various wing designs helped improve performance for a particular flight mission by altering the stability, lift, and drag at various times during a flight. Drastic improvements to the performance and efficiency of airplanes have been made due to over a 100 years of aviation technology development. Even with these improvements there are still design compromises present today. One of these being the static shape of the aircraft throughout its flight mission. This static shape limits the aerodynamic efficiency as the focus must be placed on the most important flight regime and the remaining would be considered as off design targets. The idea behind a morphing aircraft is to improve the aerodynamic efficiency at multiple flight regimes. Instead of having to compromise with a static design, an aircraft's structure would adapt according to the current objective. This thesis explores this idea with a modular morphing wing design. 


\subsection{Aircraft Morphing Design}

Although the idea of a morphing aircraft may seem new, morphing designs have been prevalent throughout aviation history. It is important to note that all aircraft have some form of morphing such as the control surfaces which change the effective shape. The type of morphing considered in this thesis report however involves more dramatic change resulting in greater potential for aerodynamic performance increase. Table $1.1[1,2]$ outlines some aircraft with major morphing structures which exhibit changes in twist, chord, span, sweep, and dihedral.

Table 1.1: Aircraft Morphing Historical Perspective [1,2]

\begin{tabular}{|c|c|}
\hline 1903 & Wright Flyer (variable twist) \\
\hline 1931 & Makhonine MAK-10 (variable span) \\
\hline 1935 & Makhonine MAK-101 (variable span) \\
\hline 1940 & Bakshaev RK-800 (variable chord) \\
\hline 1947 & Makhonine MAK-123 (variable span) \\
\hline 1951 & Bell X-5 (variable sweep) \\
\hline 1964 & North American XB-70 Valkyrie (variable dihedral wingtips) \\
\hline 1964 & General Dynamics F-111 Aardvark (variable sweep) \\
\hline 1969 & Tupolev TU-22M (variable sweep) \\
\hline 1969 & British Aircraft Corporation Concorde (variable engine air intake, pitch nose) \\
\hline 1970 & Grumman F-14 Tomcat (variable sweep) \\
\hline 1974 & Rockwell B-1 Lancer (variable sweep) \\
\hline
\end{tabular}

The above table comprises of mostly military applications as their objectives are more concentrated on maximizing performance and less on cost when compared to that of commercial aircraft. It is also important to note that many military applications comprise of more diverse flight conditions which is when morphing-wing designs are most beneficial [3].

It should be obvious that a system's complexity greatly increases by incorporating morphing technology. As the risks of failure are high, more complex systems could potentially lead to catastrophic scenarios. This is a major reason why airplanes in table 1 exhibit partial morphing where only a few parameters are chosen instead of all of the following: twist, chord, span, sweep, or dihedral. The following figure illustrates what each parameter change would resemble for a simple case. 


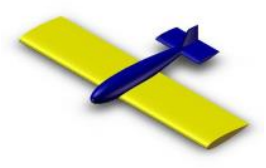

Baseline

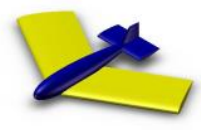

Sweep

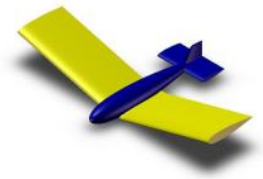

Cant

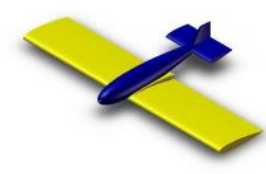

Twist

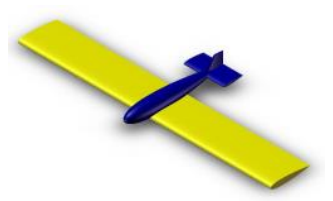

Span

Figure 1.1: Wing Level Morphing [4]

To understand the reference frame for the subsequent chapters, the following figure lists the main axes for an aircraft as used throughout this research.

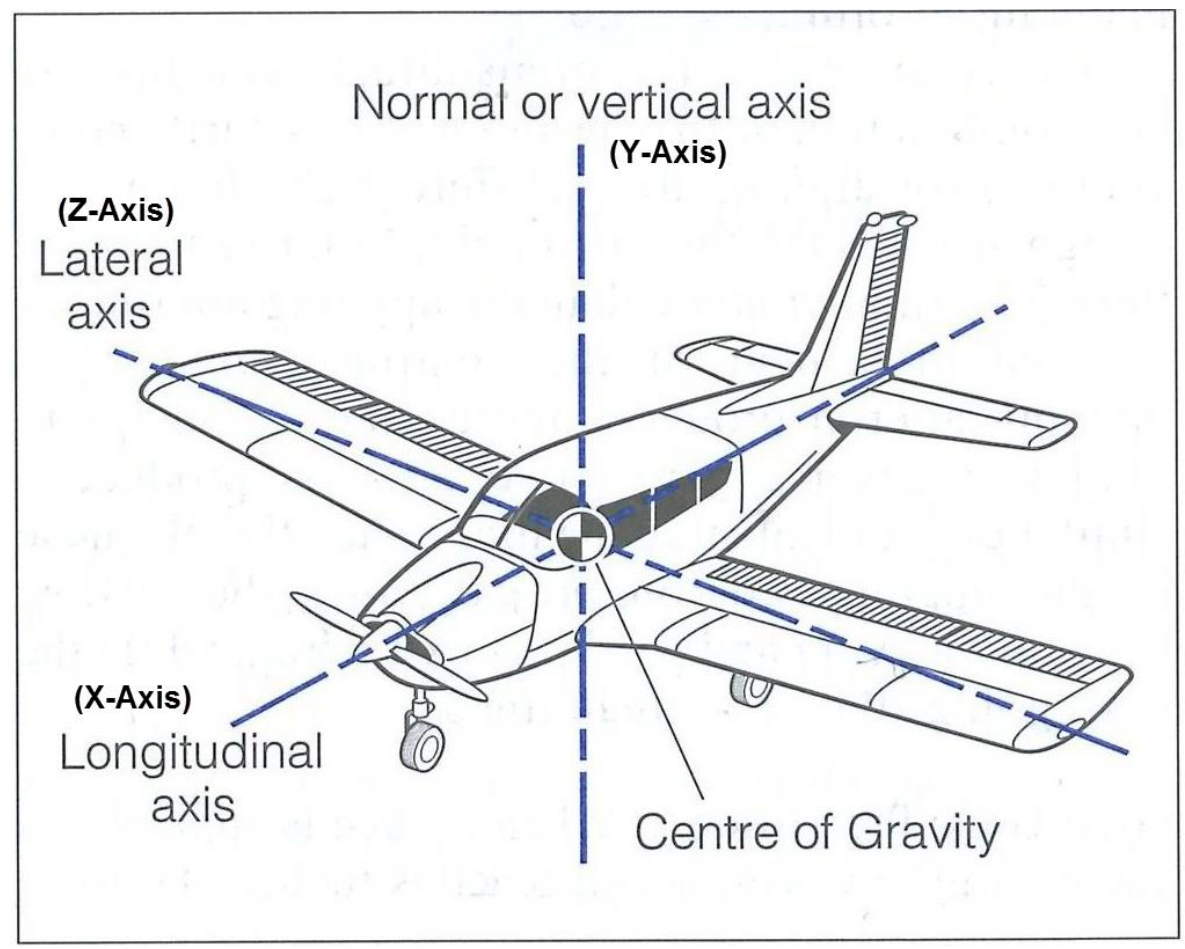

Figure 1.2: Coordinate Frame [5]

When considering figures 1.1 and 1.2 , sweep rotates on the $\mathrm{x}-\mathrm{z}$ plane, cant on the $\mathrm{y}-\mathrm{z}$ plane, and span on the $x-z$ plane. Twist is noted by the rotation about $1 / 4$ chord line of the wing from root to tip. The sweep and cant angles are in relation to the $1 / 4$ chord line.

The high risk involved for a morphing wing requires detailed research to prove that this design choice allows for significant performance increase. This would then of course lead to improvements in technology for more reliable systems. 


\subsection{Problem Statement}

Morphing wings are designed to improve overall efficiency for multiple flight regimes when compared to a static wing configuration. A static wing is limited in the sense that a compromise in performance is made so that the wing is not fully suited for less important flight maneuvers such as during climb and descent. An example of this would be the fuel consumption being a major factor for commercial airplanes which would then lead to cruise being the primary objective due to the time spent in this flight regime. A morphing wing design allows for improved performance at the off design cases such as climb and descent without jeopardizing the performance during the primary flight regime such a cruise.

An ideal aircraft would mimic the operations of birds where continuous wing adjustments would be made. Birds have the ability to change its shape into infinite amount of configurations while maintaining smooth skin curvature.

Since these attributes are extremely difficult to recreate a modular morphing wing would be a step in the right direction. Although it's not a continuous surface it allows for morphing ability without investing heavily in proprietary smart materials for adaptive skin designs.

This thesis goes into detail from start to finish for the shape design of a module discretized morphing wing suited for three flight regimes; climb, cruise and descent.

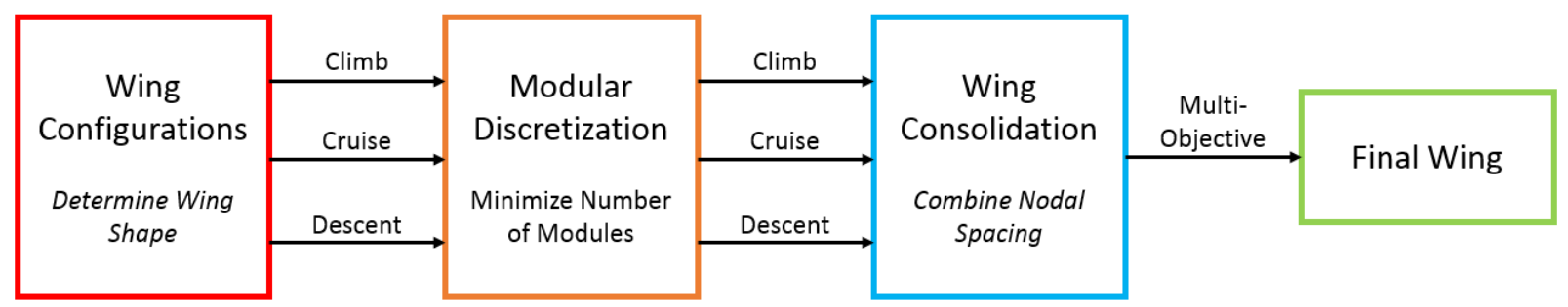

Figure 1.3: Initial to Final Wing Strategy

Due to the number of steps involved within this research, each major milestone is presented in figure 1.3. Notice the three major milestones are wing configurations, module discretization, wing consolidation, and then the outcome is the final wing where its performance is checked back with the original wing profiles. Also notice between the first three milestones 
there are three arrows with climb, cruise, and descent labels. Each arrow represents a parallel process involved where steps are being done with their respective flight regimes involved.

The advantage of dividing the research work into multiple sections is to help establish areas where the user intervenes and reviews the results before moving onto the next step. The wing configurations (wing profile) step uses an optimization routine to determine wings suited for climb, cruise, and descent flight regimes. The module discretization technique, developed by Finistauri [4], converts the wing profiles into a modular wing. These modular wings are then analyzed so that the minimum number of modules is found for each case without sacrificing aerodynamic performance. Finally the consolidation step is a new process developed by the author to combine multiple discretized wings into a single final wing based on the flight mission objectives. The end resultant wing is then compared to the original wing profiles' aerodynamic performance. 


\subsection{Thesis Outline}

Chapter 1 introduces the problem that a morphing wing attempts to solve. The basic research method is explained from a high level perspective.

Chapter 2 discusses the literature review of past work pertaining to wing profile design, modular wing discretization, and finally multi objective designs. An example is explained which goes through the procedure as outlined in figure 1.3.

Chapter 3 discusses the process of finding wing profiles suited for cruise, climb, and descent. An optimization routine is used in conjunction with a CFD solver to create these initial profiles. These profiles are then smoothed due to noise in the geometry generated from the optimization routine. This approximated smooth profile is then used as the reference wing for the wing discretization.

Chapter 4 introduces the use of Ansys workbench in the hope of more accurately capturing the aerodynamic properties. This results in longer run times however which was why it was not a viable option for chapter 3's optimization. Wing discretization is performed on the reference wing then wings with the minimum required modules for each flight regime are chosen based on the aerodynamic performance.

Chapter 5 completes the design process with the consolidation of the discretized wings into a single wing configuration. This configuration is defined by the nodal spacing of the joints between modules. The final wing can then change into configurations suited for climb, cruise, and descent.

Chapter 6 concludes the thesis with reiterating important findings such as the effects of the joint location on aerodynamic performance. Performance sensitivity of adding additional modules as well as joint location for consolidation is discussed. 


\section{Literature Review}

This chapter presents the past and current morphing aircraft technology. The three sections of this chapter relate toward topics illustrated in chapter 3 to 5 . At the end of each of the three sections within this chapter a high level description of this thesis' research methodology is description for wing profile generation, module discretization, and wing consolidation. Section 2.1 discussions past research on a wing's shape and how design various configurations affect an aircraft's aerodynamic performance. This is followed by section 2.2 where morphing wing techniques are presented including the module discretization of a reference wing. Finally the last section, 2.3, focuses more on multi objective design and the potential for morphing aircraft success which leads into the consolidation technique developed within thesis.

\subsection{Wing Shape Design}

It is known that an aircraft's ability to change into optimal configurations based on the current flight requirements has great potential to improve aerodynamic efficiency. The first step to realizing this potential is to determine the effects of various wing parameters.

The two major types of morphing wings include airfoil level morphing, and wing level morphing. Although both are independent of one another, as the airfoil level deals with the wing's 2-dimensional cross section and wing level is for 3-dimensional changes across the entire wing, this thesis focuses primarily on wing level changes. Table 2.1 displays some performance 
impacts based on various wing parameter change where majority are related to wing level morphing such as span, sweep, dihedral, and twist.

Table 2.1: Shape Parameters and Performance Benefits [6,7]

\begin{tabular}{|c|c|c|}
\hline Parameter & Increasing & Decreasing \\
\hline Wing Area & $\begin{array}{c}\text { Increased lift, decreased wing } \\
\text { loading, takeoff speed, turn } \\
\text { radius }\end{array}$ & $\begin{array}{c}\text { Increased speed, decreased } \\
\text { drag }\end{array}$ \\
\hline Aspect Ratio & $\begin{array}{c}\text { Increased spanwise } \\
\text { efficiency, range, loiter time }\end{array}$ & $\begin{array}{l}\text { Improved ride comfort in } \\
\text { turbulence, increased speed, } \\
\text { decreased parasitic drag }\end{array}$ \\
\hline Chord Length & $\begin{array}{l}\text { Increased wing area, } \\
\text { decreased aspect }\end{array}$ & $\begin{array}{l}\text { Decreased wing area, } \\
\text { increased aspect ratio }\end{array}$ \\
\hline Span Length & $\begin{array}{l}\text { Increased wing area, } \\
\text { decreased aspect ratio }\end{array}$ & $\begin{array}{l}\text { Decreased wing area, } \\
\text { decreased aspect ratio }\end{array}$ \\
\hline Sweep Angle & $\begin{array}{l}\text { Increased maximum speed, } \\
\text { longitudinal stability, } \\
\text { decreased compression drag } \\
\text { (super sonic) }\end{array}$ & $\begin{array}{l}\text { Increased lift-curve slope, } \\
\text { lateral control, decreased } \\
\text { pitch attitude while landing }\end{array}$ \\
\hline Dihedral Angle & $\begin{array}{l}\text { Increased dihedral effect, } \\
\text { spiral mode stability }\end{array}$ & $\begin{array}{l}\text { Increased roll stability, } \\
\text { maneuverability }\end{array}$ \\
\hline Twist & Wash-in: increased lift & $\begin{array}{l}\text { Wash-out: improved tip stall } \\
\text { performance. }\end{array}$ \\
\hline
\end{tabular}

As can be seen there are many factors that have an effect on an aircraft's performance. There are numerous examples for the wing level morphing where some are outlined here.

The first example incorporates variable span for a cruise missile comprised of a cylindrical body and telescoping wings. The telescoping bar within the wings allow it to retract and extend for span changes [8]. It was found that as the span was increased the lift distribution decreased thus causing significant reduction of induced drag. It was noted that as the wing extended in the lateral direction, the root bending moment increased dramatically. This was then further investigated for roll control as non-symmetric changes of the wing span was performed so that a moment was created about the longitudinal axis. Although the roll control was more suited for the cruise missile than a larger aircraft, the reduced drag with span increase resulted in increased range. An actual applied example of a telescoping wing was the German fs-29 which used the variable span to improve soaring performance [9]. This was defined as achieving greater 
distance in a short amount of time. Since the fs-29 was a sailplane, the aircraft has a limited window for optimal performance but variable span allowed for improved performance with a wider range of flight speeds.

Designing the option for variable sweep was thought of since a Germany patent was filed in 1924 [10]. A popular example of a successful airplane with variable sweep is the Bell X-5 from the 1950s. The Bell X-5 allowed for swept wings at 20, 50 (baseline), and 60 degrees as shown in figure 2.1 .

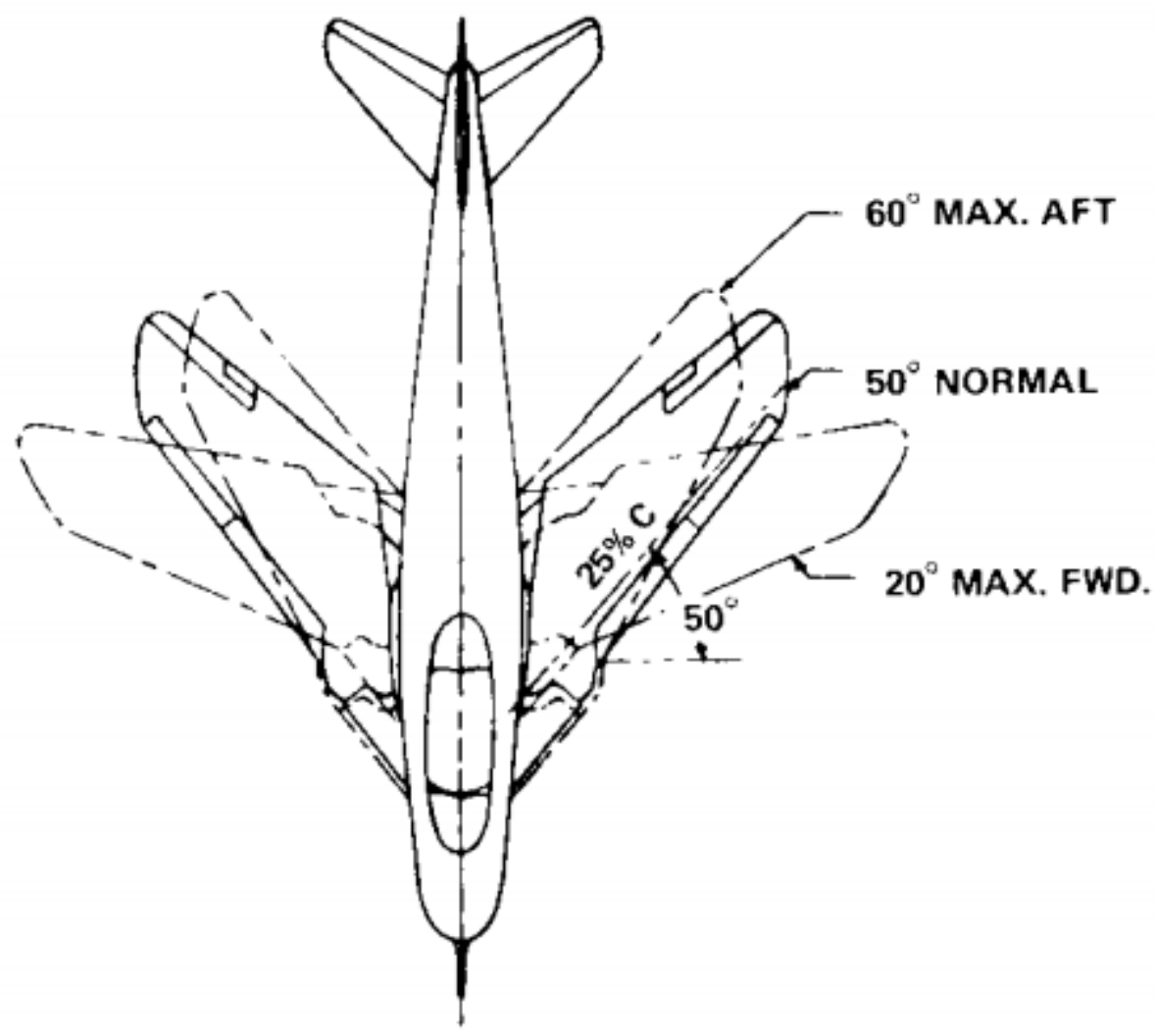

Figure 2.1: Bell X-5 Swept Arrangement [11]

It is important to note that many variable swept wing geometries were changed on the ground and not during flight due to reliability at the time. The Bell X-5 however was capable of adjusting its sweep during flight.

Variable cant was another morphing method which allowed for a number of dihedral distributions. The following figure illustrated an example used by Abdulrahim et al [12] where a micro aircraft equipped with a modular wing varied the cant angle. 

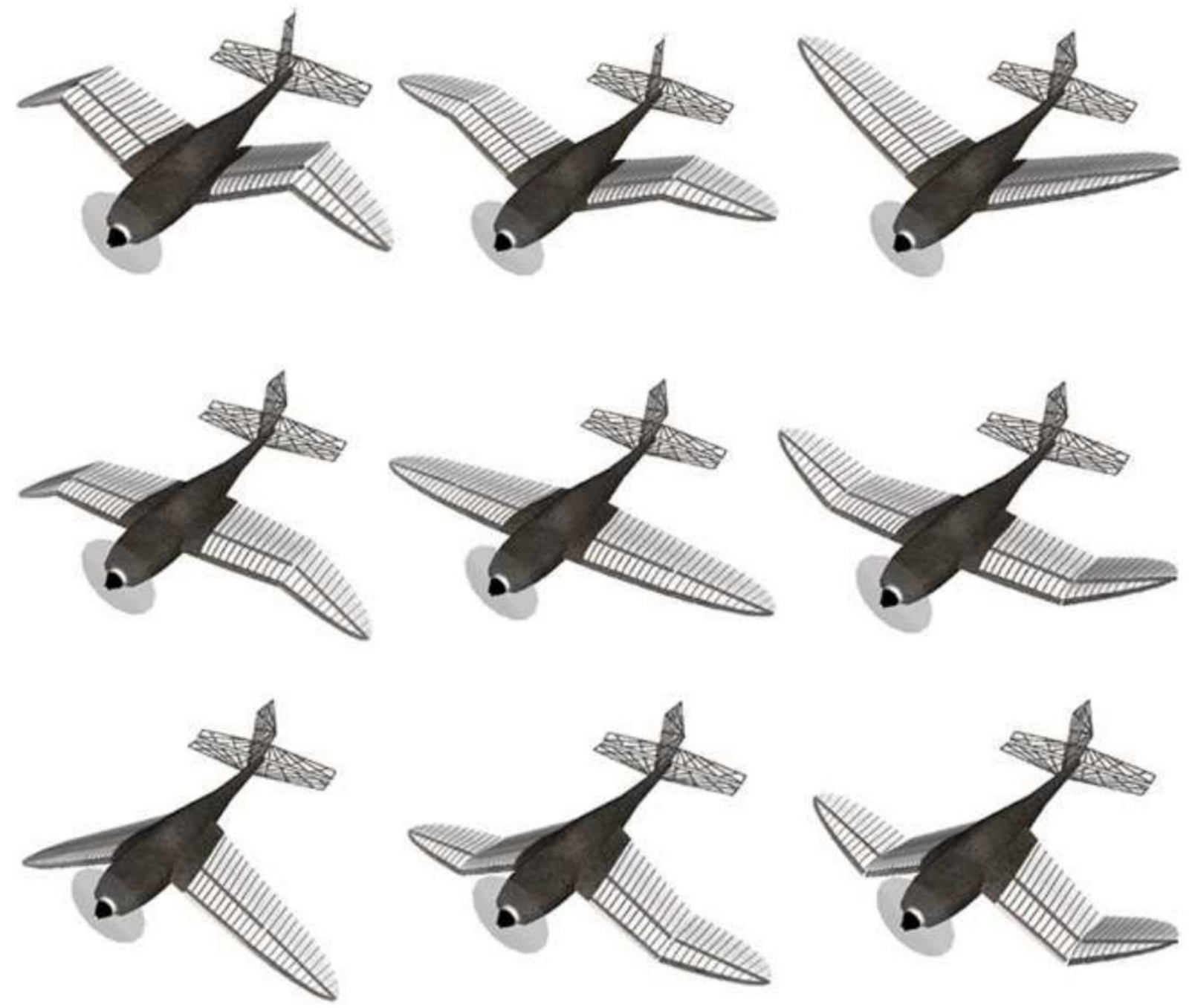

Figure 2.2: Variable Gull-Wing Morphing Aircraft [12]

These configurations were used to find optimal morphing angles for cruise flight, maneuvering, steep descent, and sensor-pointing. Maximum lift to drag ratio was achieved through an anhedral configuration to maximize the lift to drag ratio for higher aerodynamic efficiency and endurance for the cruise flight regime. Lower lift to drag ratio for steep descent objectives was also achieved by increasing both modules' cant angle which caused a decrease in the planform area [12]. Although this was not the only gull-wing design as the biological characteristics of birds have been analyzed, this research performed an in-depth study of the aerodynamic performance at multiple flight regimes with varying cant angles. This in contrast to other research which studied the aerodynamic performance for maneuvers specific to birds [13]. 
An example of an aircraft which exhibited changes in the wing area, span, chord, and sweep by 51\%, 36\%, 110\%, and 30 [degrees] was developed by NextGen Aeronautics Corporation $[2,14]$.

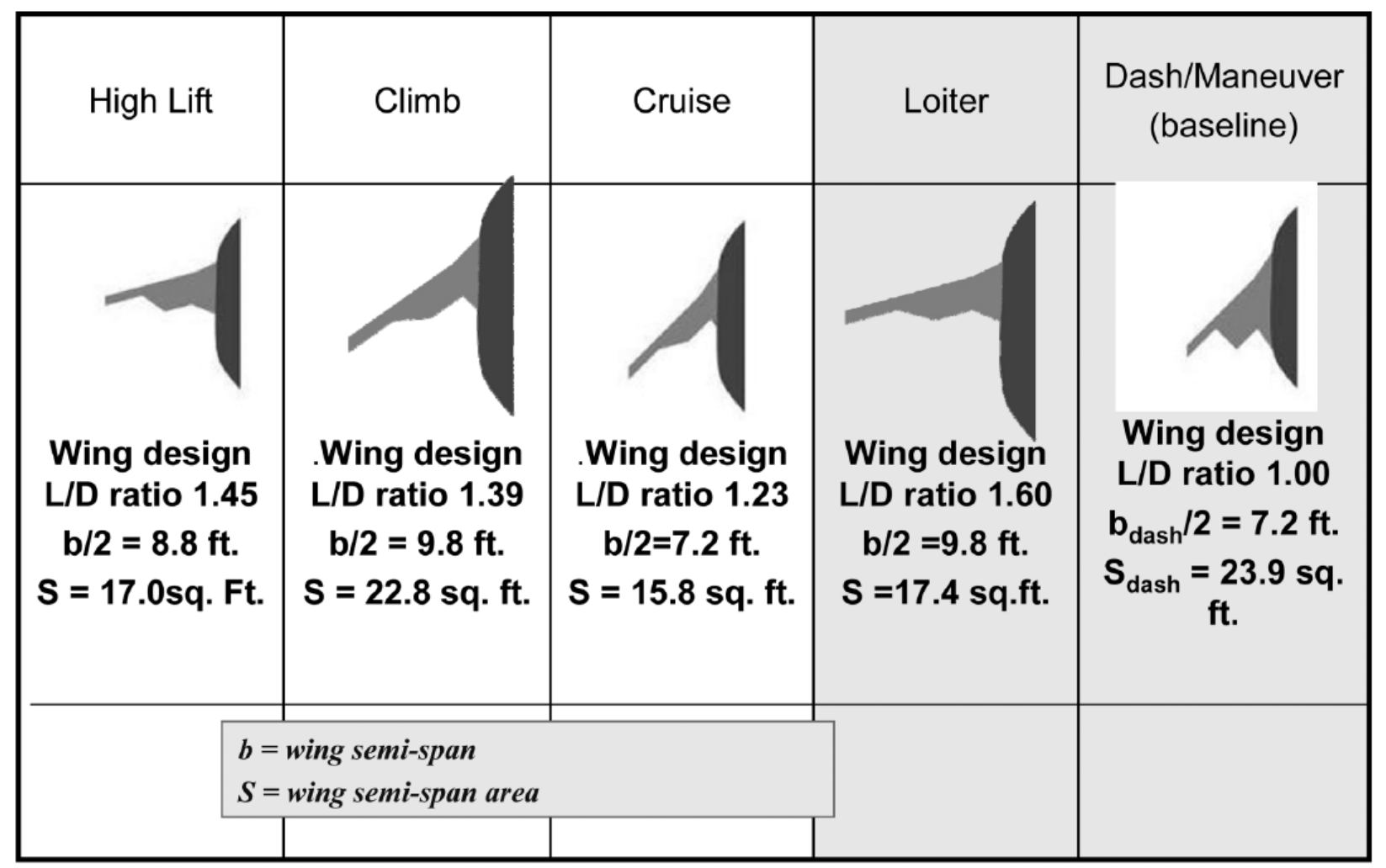

Figure 2.3: NextGen Morphing Wing Concept [2]

The design will be discussed in sections 2.2 and 2.3 but notice in figure 2.3 the changes in lift to drag ratio as multiple parameters varied especially from the baseline to loiter case where the wing is fully deployed. It is interesting to note that as discussed previous, multiple benefits can be seen by varying a single parameter but large benefits can also be seen from more dramatic changes to the wing geometry [15]. This is an important note as the improved aerodynamic performance helps justify the added complexity of changing multiple parameters.

\subsubsection{Wing Profile Methodology}

This section illustrates the methodology to develop a wing profile for a given flight regime. This example will be continued in sections 2.2.1 and 2.3.1 which discuss module discretization and consolidation, respectively. 
The first step involves finding wing configurations suited for a particular flight regime. The following example illustrates the steps involved. Imagine two flight regimes noted by A and B. The first step would be to find the performance index for each flight regime. Since this research deals with aerodynamic performance, an index example could be the lift to drag ratio.

The performance index is used as the objective function for an optimization routine. This would include many fluid simulations each with varying wing geometries, then logically altering the geometry with the aim of scoring a higher performance index value (see section 3.1.1). As noted previous, aerodynamic performance changes drastically with adjustments made to wing parameters: sweep, cant, twist, and span. This example shows only that of the cant change (dihedral distribution) from root to tip.

Once the optimization routine is complete, the end result can resemble that of figures 2.4 and 2.5. These figures show the wing's location in space along the span vs the aircraft's normal (vertical) axis, thus the curve represents the wing's dihedral distribution.

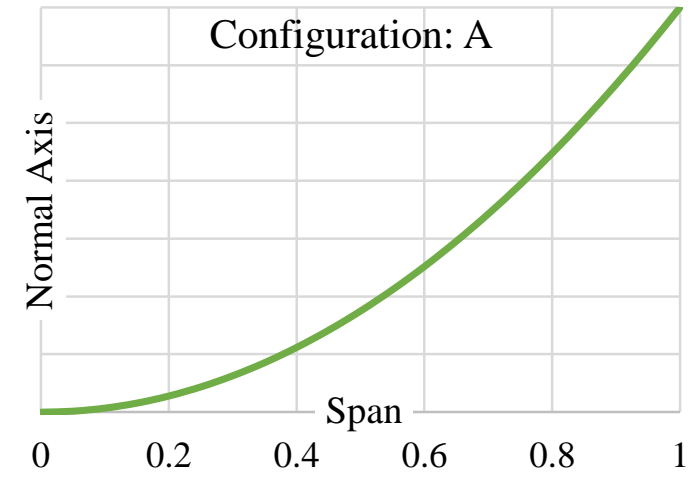

Figure 2.4: Example A Configuration

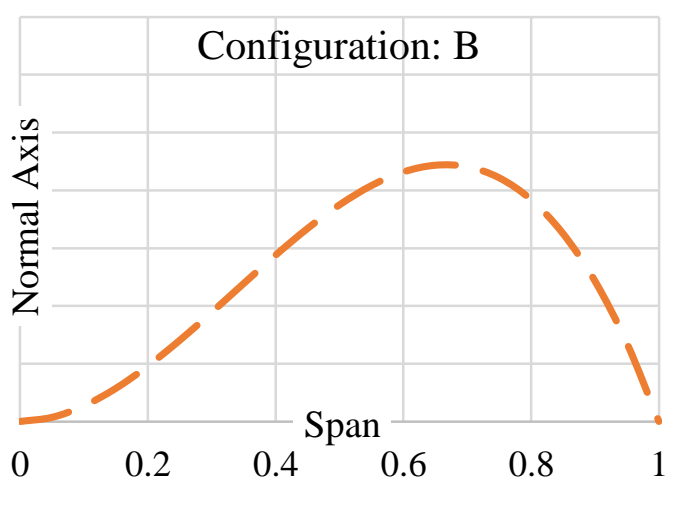

Figure 2.5: Example B Configuration

The span scale is from root to tip, which is denoted by 0 to 1 . The important aspect of the above figures is the shape of the dihedral distribution from root to tip and how they differ from each other so no units have been presented for the normal axis. If these configurations are deemed acceptable than the module discretization can begin. 
Although adjusting a single wing level parameter is similar to the literature review discussed previously, the actual case study in chapters 3, 4, and 5 have the freedom to vary cant, sweep, twist, and span simultaneously. The optimization routine based on aerodynamic performance allows for full control over the design space. This allows for a greater potential in aerodynamic performance especially when compared to the examples mentioned previously in section 2.1.

\subsection{Morphing Wing Techniques}

Two main types of wing morphing techniques involve either using smart materials for adaptive skin that can not only withstand load but also change its configuration according to the situation. This could be as elaborate as sensing various fluid parameters to activate a stimulus to change the wing geometry [1]. The other morphing wing technique is that of a modular wing where linear segments are combined to create a discretized wing which are controlled by actuators $[4,16]$. One example of this is the NextGen Aeronautics Morphing UAV where the wing would rotate outward to from the fuselage effectively changing the span and wing area [1].

Many morphing wing concepts involve a combination of an adaptive skin made of smart materials and that of a modular wing [17]. The following examples will highlight more on modular wings where morphing occurs primary from mechanical structures.

The NextGen morphing wing concept, shown in figure 2.3, is a bat-like wing for high lift, climb, cruise, loiter, dash/maneuver as baseline. Adjusting sweep and span is possible due to hydraulic actuators and tubular design for the leading edge [12]. Although the wing can be suited for multiple flight regimes, this concept seems more about capitalizing on the available wing geometry changes itself and making the best of this situation. This is a clever design but the limitations of the structural changes is apparent as the wing parameters are interrelated. An example is that the span cannot change without adjusting the sweep angle.

Another morphing wing aircraft is the Lockheed hunter killer morphing aircraft, where the wing folds inward toward the fuselage as seen in the following figure. 

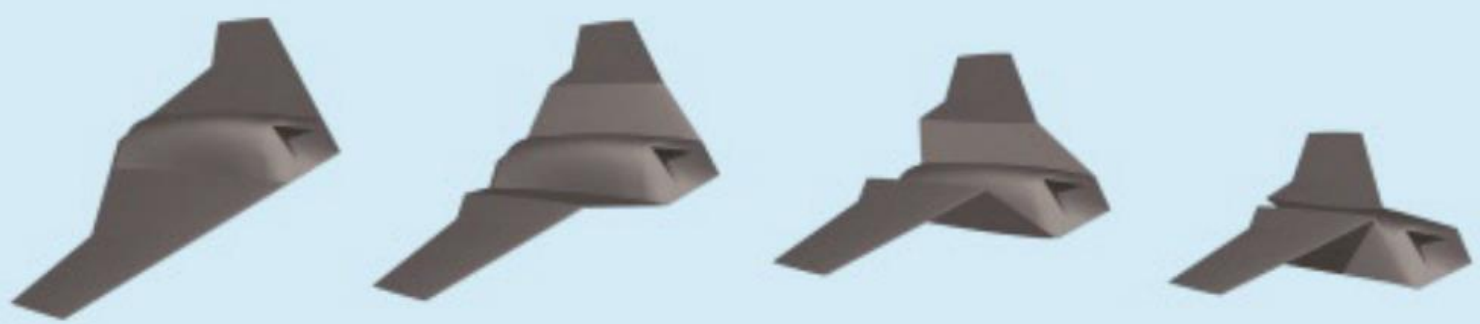

Figure 2.6: Lockheed Martin Z-Wing [17]

This concept does not provide ideal shapes but instead to provide performance gain dictated by cost and risk [18]. This approach again deals with developing a structure capable of shape change and then using the resultant aerodynamic benefits for various flight regimes. The performance gain is still dramatic however due to the simplicity of design thus reducing the structural complexity, weight, and energy to morph the wing structure.

Although both the NexGen the Lockheed Martin examples are a few of many these cases proved to be very promising in terms of aerodynamic performance and simplicity of design. A method was developed by Finistauri et al [19] where a reference wing is discretized into linear segments which results in a modular wing. Although the complexity of the design is much higher than that of both the NexGen and Lockheed Martin's attempts, the design space for possible configurations is greatly increased. This design allows for reference wings with variable sweep, cant, twist, and span adjustments so that the module discretized configurations can adjust each module with respective to the adjacent one. A similar concept can be seen in figure 2.2 where the wings were made of linear segments.

Past research using the discretization technique for a modular wing was used for a wingtip design [16]. The following figure shows some of the results from the wingtip research. 

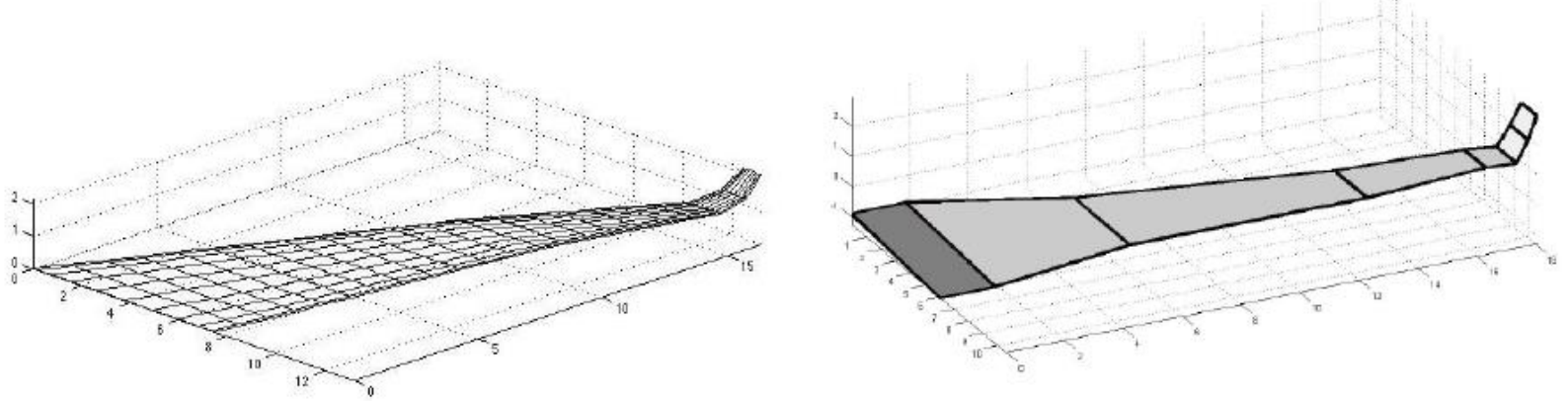

Figure 2.7: Discretized Wing Tip Optimization [16]

Notice the wing modules is concentrated around the wingtip region. Figure 2.7 is shown as an example but it is important to note that more modules are placed near the wing tip where larger wing curvature occurs.

As a continuation of section for wing profile methodology (section 2.1.1), section 2.2.1 highlights the steps taken for wing level discretization from a reference wing so that a modular wing is created. This method is particularly important as it allows for any reference wing previous optimized for a given flight regime to be discretized into a modular wing. This avoids limitation the design exploration.

\subsubsection{Module Discretization Methodology}

Once the configurations from section 2.1.1 were determined, the next step is to discretize the reference wing into modular segments. One method is to simply divide the wing into equal parts. The major goal of this step however is to minimize the total number of modules thus we must take a slightly different approach. Using the wing discretization method developed by Finistauri et al. [19] (see section 4.2), individual module sizes can be determined based on the wing's curvature. This allows shorter modules to occupy space with larger wing curvature and longer modules for areas with smaller wing curvature.

Once the configurations are divided into multiple segments, aerodynamic analysis would then help determine the minimum number of modules necessary without significant decrease in the performance index. The results may look like that of figures 2.8 and 2.9. 


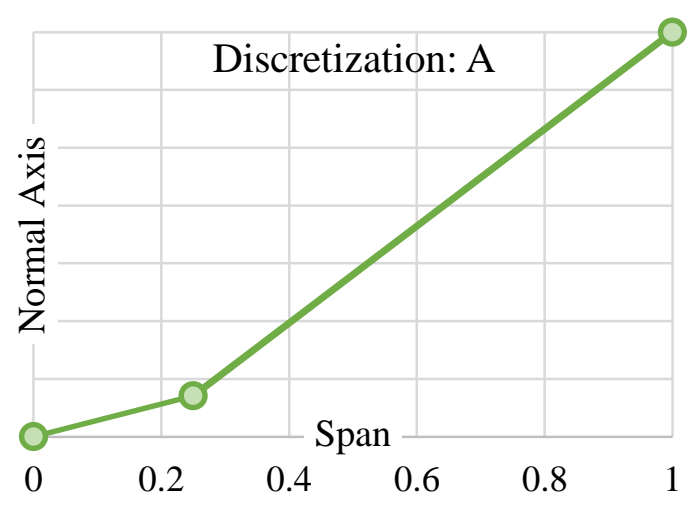

Figure 2.8: Example A Discretization

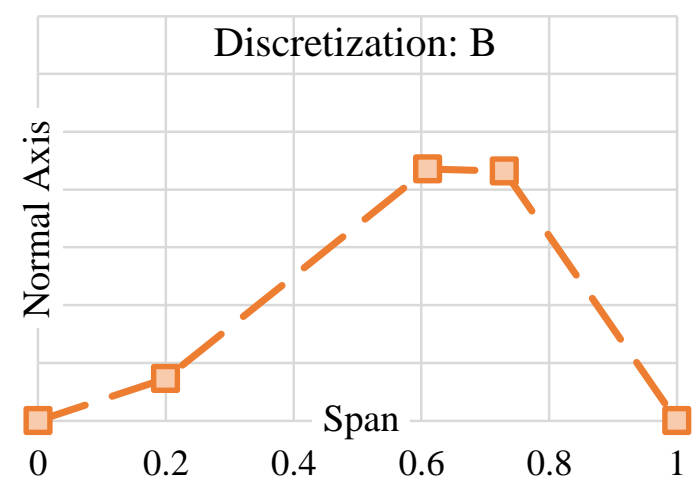

Figure 2.9: Example B Discretization

Note the circle and square points in figures 2.8 and 2.9 represent the nodal points between modules. The straight sections represent the actual modules. As can be seen configuration A only needed 2 modules while B required 4 due to the added complexity. Once the configurations are divided using the minimum number of modules, the consolidation into the final wing can begin.

\subsection{Multi Objective Design}

As mentioned a morphing wing must be capable of adapting to various flight regimes in order to be effective. A study has been done to study a wing's geometry change with that of the flight regime of a normal aircraft's flight mission [20]. This includes aspects such as cruise and climb. The BQM-34 Firebee UAV was used as the baseline where changes to the airfoil level and the wing level was explored for separately for various flight regimes. It's interesting to note that the wing level morphing outperformed the airfoil level in nearly all flight regimes as can be seen in the following figure. 


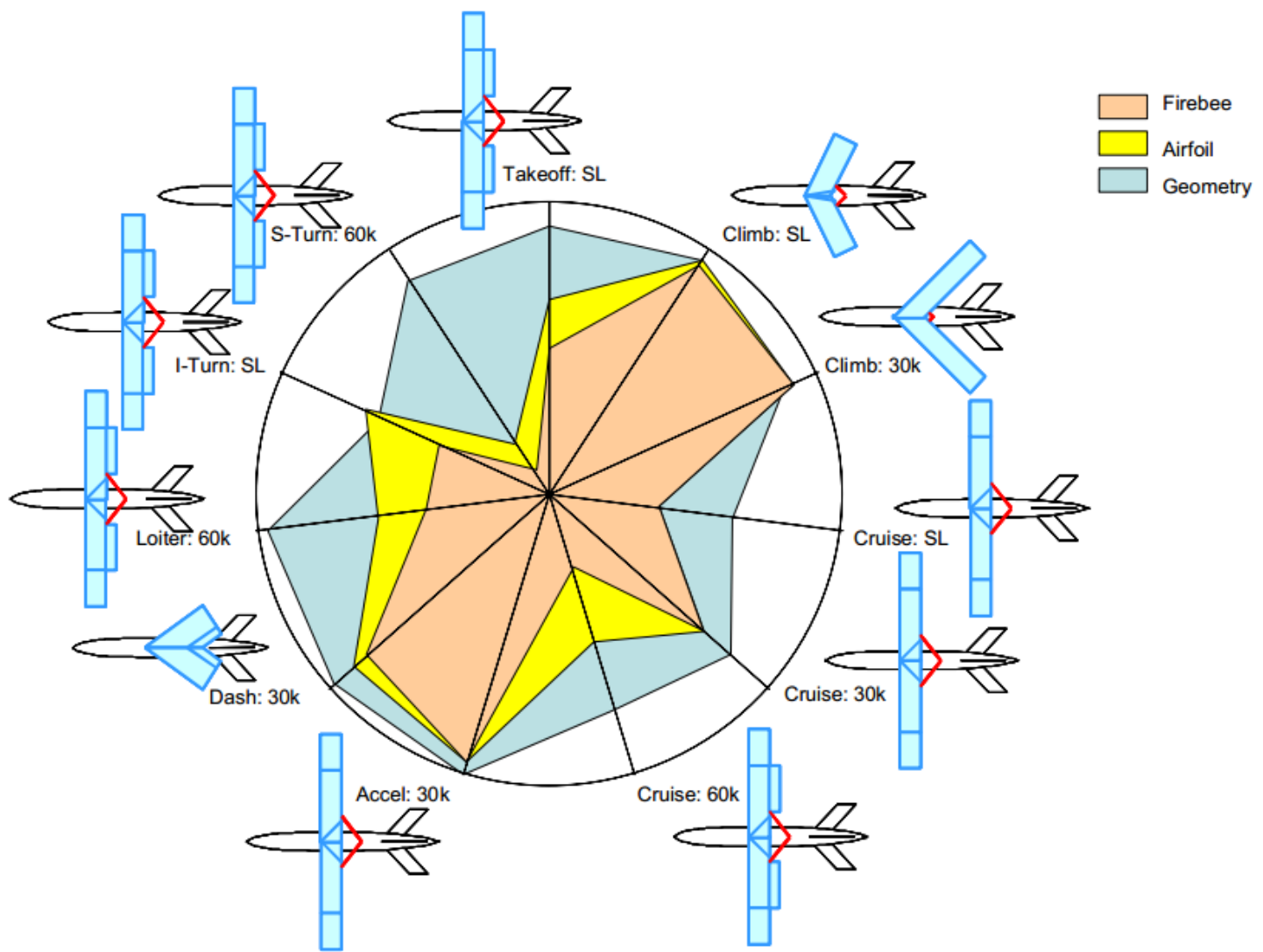

Figure 2.10: Performance of Firebee Baseline vs Morphing Wings [20]

Figure 2.10 only shows the relative performance between the Firebee baseline, airfoil morphing, and wing level morphing. Each line extending from the center of the circle to the outer edge represents a different flight regime analyzed by Joshi et al [20]. The airplane shapes were the resultant wing configuration changes for their respective flight regime. The orange coloured sections represent the performance values for the Firebee baseline case which has no changes to airfoil and wing configuration. The yellow and green areas present the relative performance for airfoil and wing morphing. The coloured sections are relative for the sake of comparison thus no values are shown in figure 2.10. The better performance values is represented by the coloured section that extend toward the outer edge where lower performance are shown by being closer toward the center of the circle. This plot is important as it shows the potential for performance improvements in nearly all flight regimes with wing morphing technology. 
Smith et al [3] discuss the flight segments for a fighter jet and how a morphing wing would improve performance. This study approximated the benefits of using a morphing wing by estimating fuel penalties involved for morphing mechanisms and the power to actuate these morphing sections. It was found that even with large fuel penalty estimations the morphing wing still was able to outperform the fixed wing case where a 5-30\% improved in fuel consumption was estimated. It's important to note that the main benefits were found during the subsonic flight segments of the mission. This was because the fixed wing case were primarily optimized for supersonic flight but the morphing wing allows the wing to change into a configuration more suited for subsonic flight when needed. Although the authors mention more research needs to be done to properly estimating the fuel costs of adding morphing mechanisms, the benefits even at highly over estimated fuel penalties shows great potential. The benefits at subsonic flight segments shows that the most benefits are founded during the aircraft's off design which is as expected for a morphing wing. This highlights that even if a morphing wing doesn't outperform the baseline case at all configurations, the overall performance increases throughout the entire flight mission.

The module discretization method discussed in the previous chapter developed by Finistauri et al. [19] was designed for a single reference wing. To apply this to multiple flight regimes a method must be developed that extends the module discretized wings into a consolidated wing where choosing a wing with minimal number of modules is still a primary focus. The module discretization technique is based on wing curvature instead of simply dividing the wing evenly span-wise so that the minimum amount of modules can found, the consolidation technique cannot simply add joint locations into a single wing as closely aligned joints can be combined for further consolidation. A high level explanation is explained in section 2.3.1 which completes the methodology example shown in sections 2.1.1 and 2.2.1. Refer to chapter 5 for an in depth description of the consolidation technique.

\subsubsection{Wing Consolidation Methodology}

Wing consolidation mentioned here is the next step from the module discretization of multiple wing configurations. This would be the final step for morphing wing shape development from a purely aerodynamic point of view. Simply combining several module discretized wings 
together would likely add unnecessary modules, thus a consolidation technique continue to reduce the number of total modules but combining closely located joints without significant impact on aerodynamic performance.

As a morphing wing's main advantage is to adjust its shape based on a given situation, thus the two separate wings in this example, A and B, must be combined into a single wing. As mentioned simply combining joint locations from multiple wing configurations can create additional modules that are not necessary. A solution is to perform wing consolidation on the combined wing as shown in the following figure.

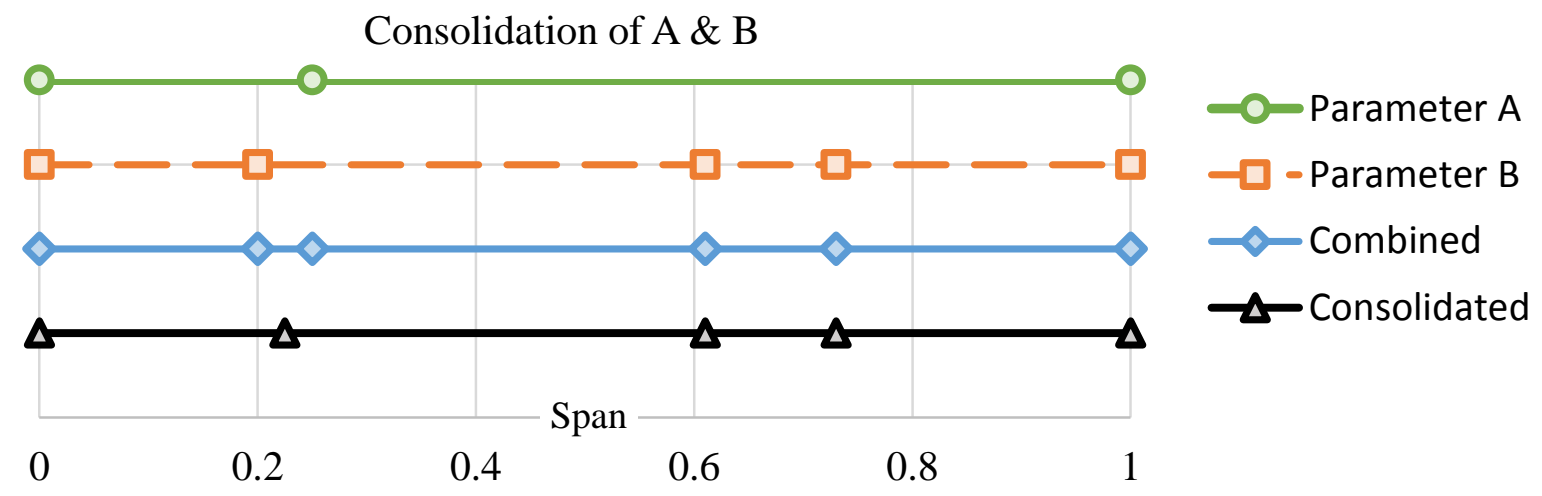

Figure 2.11: Example A \& B Consolidation

Figure 2.11 shows this process where the summation of nodal spacing forms the 'combined' line. The combined line has 5 module segments but notice the nodal points at close to 0.2 span are close together. Further consolidation can be made so that these two joints are combined into one. The idea is to perform a sensitivity study so the relationship between the performance index and the exact location of the joint can be understood. This would then allow a weighting function to be added so that primary flight regimes would have precedence over other secondary flight regimes. If an aircraft's overall flight objective is to improve fuel consumption then the flight regime that absorbs the most fuel would receive the highest weight. Once this step is complete the final wing would be known as shown in the following figure. 


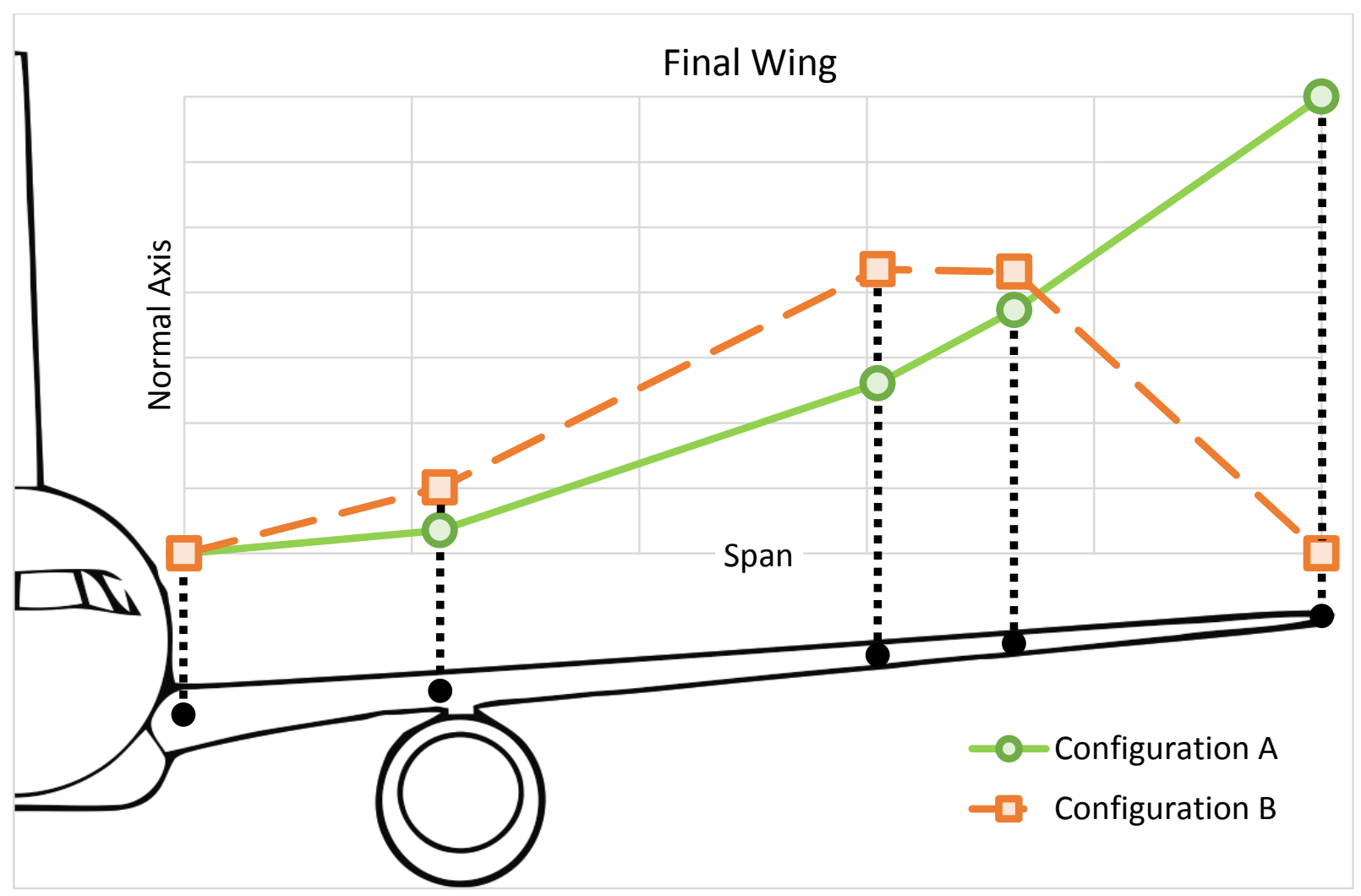

Figure 2.12: Final Wing Example

Figure 2.12 shows the two wing example configurations layed over simple aircraft's wing. Notice the nodal spacings are common in all wings. The picture of an airplane could represent a third configuration that has a constant dihedral. The final step is to check how much the final wing configurations in figure 2.12 deviated in performance from that of the initial wing profiles from figures $2.8 \& 2.9$. This is just a confirmation that the overall procedure of module discretization and consolidation did not significantly affect the performance index of the original wing profiles. 


\subsection{Assumptions and Limitations}

It should be noted the assumptions made for this particular thesis work. First is this research only concerns the aerodynamic analysis at steady state conditions. No transient analysis is done especially with morphing wings in motion. All analysis is done at each wing configuration as if the wing was already fixed in the respective orientation.

Structural components or weight consideration are ignored in this section. Although much effort is made to reduce the number of modules, the direct benefit of this in terms of structural complexity or weight savings is not considered.

The primary aerodynamic performance calculated is the coefficient of lift and $\operatorname{drag}\left(\mathrm{C}_{\mathrm{L}} \&\right.$ $\mathrm{C}_{\mathrm{D}}$ ) as it relates directly to the performance indices for particular flight regimes. Any performance parameters relative directly to a particular aircraft are ignored such as specific fuel consumption. Although parameters such as rate of climb and range are considered, this is in a general sense and no absolute values are assumed.

The three flight regimes that are considered are for climb, cruise, and descent. These are not fixed to any particular aircraft but instead the general fluid conditions. The importance is again not to concentrate on particular performance values in their absolute form but instead the aerodynamic trends that occur with wing shape change. Ground effects are also neglected in particular for the climb flight regime.

The fluid is assumed to be standard sea level conditions. The air free stream velocity is set to $0.3 \mathrm{Mach}$ which allows for incompressible fluid flow assumption. Reynolds number of 4683000 which is suitable for small aircraft. The root chord was set to $1 \mathrm{~m}$ and approximate span about $3 \mathrm{~m}$, this changes per wing configuration. The taper ratio was set to 0.1 and the airfoil set to sc20518 for a more efficient wing design. 


\section{Wing Profile}

This is the first step of the case study where the initial wing configurations are found using an optimization routine with three flight regimes in mind, climb, cruise, and descent. The formation of the performance objectives for each flight regime and the resultant wing profiles generated are outlined here. The objective here is not to find the best optimal wing configurations but instead to have wing profiles that have relatively good performance at their respective flight regimes. These wing profiles were then used in future chapters for discretization and consolidation.

\subsection{Computational Methods and Conditions}

\subsubsection{Optimization Routine}

A wing shape optimization must be done to determine suitable wing configurations for each of the three flight regimes, cruise, climb, and descent. These wing configurations will be used as the initial wings, and after some smoothing, will be used as the reference wing needed for the wing discretization. The optimization routine chosen is the harmony search algorithm. Details of this method can be found within the referenced 'A New Heuristic Optimization Algorithm: Harmony Search’ paper by Geem et al. [21].

It is important to note that the resultant wings from the optimization procedure are composed of 25 modules. This was chosen instead of a smooth wing so that the design space can be quantified where each module is free to change sweep, cant, and twist relative to the adjacent 
modules as well as limited span length changes. This allows the user to have reasonable control in the parameter limits which in turn helps minimize the design space without significantly sacrificing the freedom to change into various geometric shapes. Choosing 25 modules for this step does not have any particular significance and was chosen so that it closely resembles a smooth wing. Since 25 is a large amount of modules, this helped minimize the aerodynamic performance differences when compared to a smooth wing which was confirmed in section 3.5. The parameter changes were along the $1 / 4$ chord point for the outer side of each module (side closest to the wing tip). Since there are 25 modules and 4 degrees of freedom for each, there are 100 design variables resulting in a large design space. Although the specific limits would change as per case since different performance objectives would require greater freedom with certain parameters, the general limits for each module were

Table 3.1: Harmony Search Algorithm Parameter Limits

\begin{tabular}{|c|c|c|c|c|}
\hline Limits & Sweep & Cant & Twist & Span \\
\hline Min & $-15^{\circ}$ & $-10^{\circ}$ & $-2.5^{\circ}$ & $-15 \%$ \\
\hline Max & $15^{\circ}$ & $10^{\circ}$ & $2.5^{\circ}$ & $+15 \%$ \\
\hline
\end{tabular}

\subsubsection{Computational Fluid Solver}

The fluid solver chosen for this section was an open source Matlab code using the vortex lattice method called Tornado developed by Melin et al [22]. This fluid solver was chosen due to the relatively high accuracy and short run time. Also since it was open source the code was easily modified to incorporate the optimization routine. Although the vortex lattice method has the assumption of inviscid flow which could cause issues with accurately calculating drag, this method was suitable for the initial wing profile generation. Analysis in chapters $4 \& 5$ involve a finite element method computational fluid dynamic software to accurately account for inviscid effects.

The flow conditions were set to standard sea level where the free stream velocity was set to $0.3 \mathrm{Mach}$. The angle of attack for all cases was set to $3^{\circ}$. Although using the same Mach number for all three flight regimes is not ideal, 0.3 Mach was chosen as it resembles between that of climb, cruise, and descent conditions. If one were to apply this methodology to an actual aircraft the Mach number would be different for each flight regime. Although any changes to the 
flow conditions, such as Mach number, would result in different reference wings from the optimization procedure this was not necessary for this thesis as the main focus was to establish the methodology for shape generation of a modular morphing wing.

The number of panels chosen for each case was roughly 18 chord-wise and 75 span-wise. Since the optimization routine allows for 25 span-wise modules, each module was made of 3 panels in the span direction.

\subsection{Performance Indices}

The three regimes include climb, cruise, and descent where rate of climb, range, and sink rate were chosen as the respective performance criteria. The following highlights the equations for the respective performance criteria where a performance index retrieved and used as the harmony optimization objective. The equations to follow were derived with the assumption of a jet-propelled airplane. Although changes with thrust and weight may occur for various flight regimes, these effects were neglected.

The following is the general formula for rate of climb [23],

$$
R / C=V_{\infty}\left[\frac{T}{W}-\frac{1}{2} \rho_{\infty} V_{\infty}^{2}\left(\frac{W}{S}\right)^{-1} C_{D, 0}-\frac{W}{S} \frac{2 K}{\rho_{\infty} V_{\infty}^{2}}\right]
$$

Where $R / C$ is the rate of climb, $V_{\infty}$ is the free stream velocity, $T$ is thrust, $W$ is aircraft weight, $\rho_{\infty}$ is the free steam air density, $S$ is the planform area, $C_{D, 0}$ is the zero-lift drag, and $K$ is the drag due to lift. In our analysis, the thrust and weight (see section 2.4) can be neglected which leaves the last two terms influencing the rate of climb which are influence by $C_{D, 0}$ and $K$. Thus minimizing both the zero-lift drag and drag due to lift, or simply minimize the total drag coefficient $\mathrm{C}_{\mathrm{D}}$, would result in the highest rate of climb.

The general equation for maximum range during cruise is given below [23],

$$
R=\frac{V_{\infty}}{c_{t}} \frac{L}{D} \ln \frac{W_{0}}{W_{1}}
$$

Where $R$ is the range, $c_{t}$ is the thrust specific fuel consumption, $L$ is the lift force, $D$ is the drag force, $W_{0}$ and $W_{1}$ is the weight of the aircraft where 0 and 1 denote the weight at the beginning and the end of the flight (change is primarily due to fuel consumption), respectively. 
Since we are dealing with aerodynamic shape optimization, $c_{t}, W_{0}$, and $W_{1}$ are neglected. The lift to drag ratio thus has the greatest impact on and aircraft's range. Therefore $C_{L} / C_{D}$ must be maximized to increase the range performance.

The following is the general sink rate equation, [23]

$$
V_{V}=\sqrt{\frac{2}{\rho_{\infty}\left(C_{L}^{3} / C_{D}^{2}\right)} \frac{W}{S}}
$$

Where $V_{v}$ is the sink rate. Using similar assumptions as previously stated, the performance index to minimize the sink rate for the descent configuration is to maximize $\mathrm{C}_{\mathrm{L}}^{3 / 2} / \mathrm{C}_{\mathrm{D}}$

Thus using the information presented here, the following are the performance indices used for each flight regime.

Table 3.2: Performance Indices at Three Flight Regimes

\begin{tabular}{|c|ccc|}
\hline \multicolumn{2}{|c}{ Cright Regime } & Climb & Descent \\
\hline Performance Index & Maximize: ${ }^{C}{ }_{L} C_{D}$ & Minimize: $C_{D}$ & Maximize: ${ }^{C_{L}^{1.5}} / C_{D}$ \\
\hline
\end{tabular}

\subsection{Initial Wing Configurations}

Now that the performance indices have been determined, the initial wing configuration can be found using the harmony search algorithm in conjunction with the vortex lattice solver, Tornado, (see section 3.1).

Since the optimization routine uses 25 modules to approximate a smooth wing and allowing for a large design space exploration, further smoothing is necessary in future steps (see section 3.4).

While maintaining constant flow conditions for Tornado, the harmony search algorithm adjusts the sweep, dihedral, twist, and span for each of the 25 modules. This continues until the best 30 wing cases converges by having small deviation in the performance index. It is important to note that a lot of research can be done surrounding the design exploration (see section 3.6) but 
for the purpose of this research, a wing configuration with improved results at a particular flight regime is deemed acceptable to continue with the discretization and consolidation steps.

After each case was run through approximately 50,000 iterations the following wing configurations were found which are shown in figures 3.1, 3.2, and 3.3 for climb, cruise, and descent, respectively. It should be noted that the profile shape for descent was found by Paudel using the same optimization routine in Matlab and performance index discussed previously $[24,25]$.
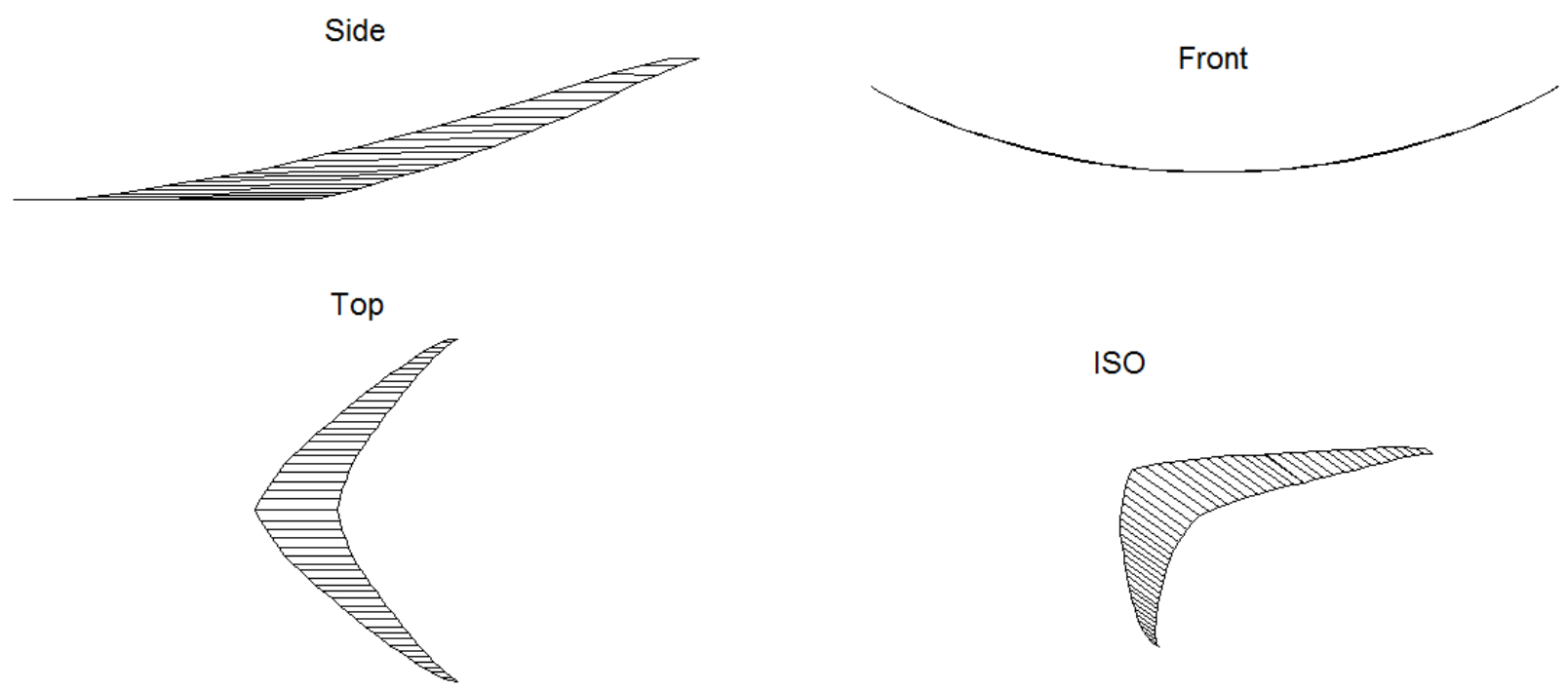

ISO

Figure 3.1: Climb Initial Configuration
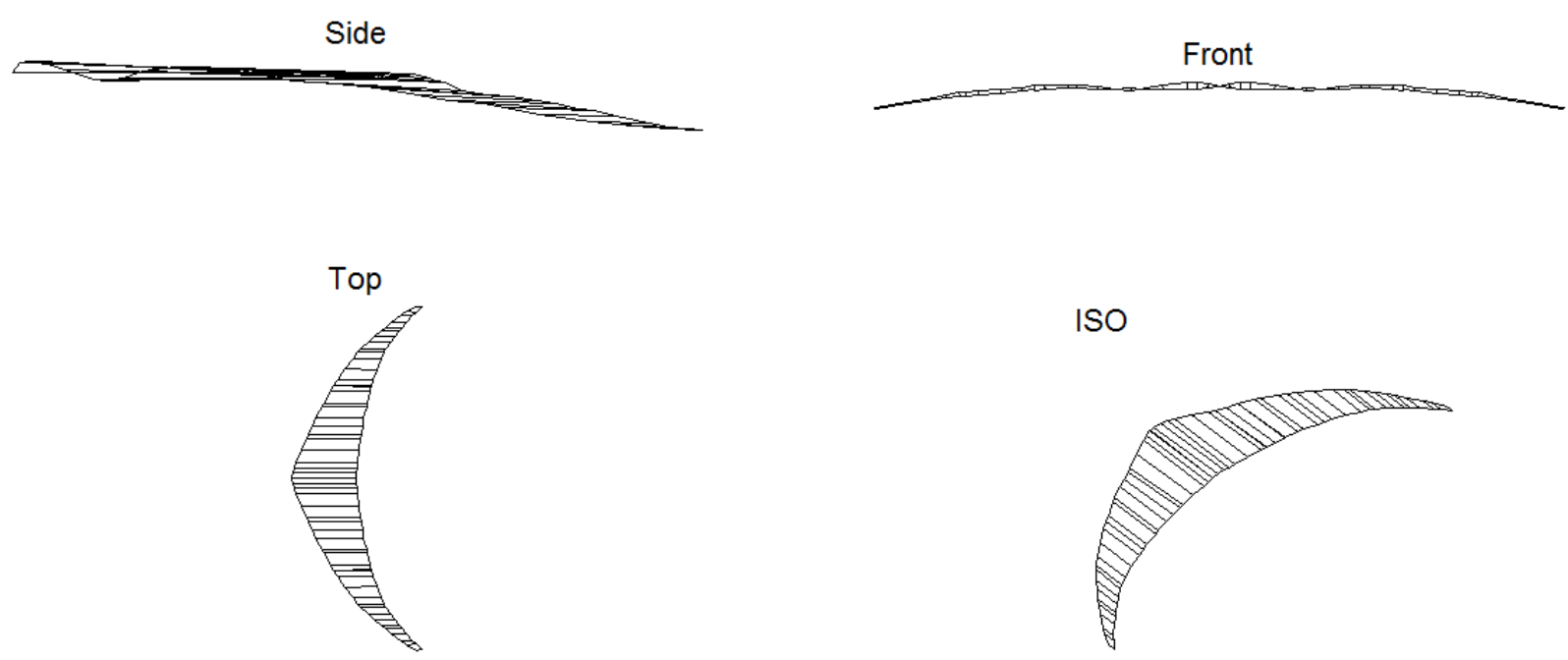

ISO

Figure 3.2: Cruise Initial Configuration 


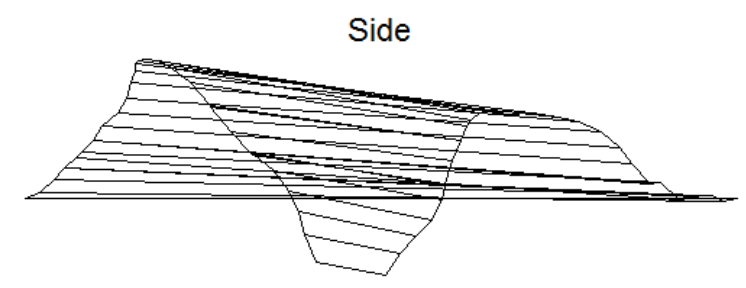

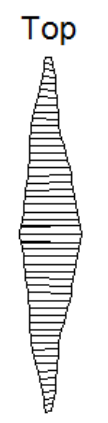

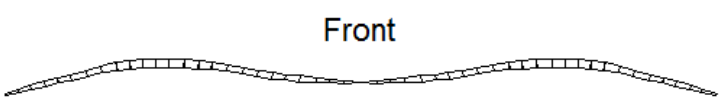

ISO

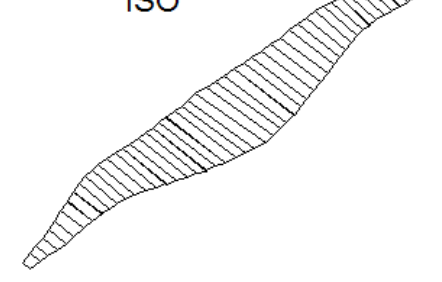

Figure 3.3: Descent Initial Configuration

It should be noted the climb configuration (see figure 3.1) has a high sweep and dihedral distribution which would contribute to the minimizing drag objective. Clearly the high dihedral distribution was also a result of not having the lift performance part of the performance index as lift did not play a significant role in increase the rate of climb. The cruise configuration in figure 3.2 exhibits a lower dihedral distribution so that greater lift can be generated. Also notice the small frontal profile and sweep near the tip of the wing which would help with minimizing drag thus increasing the lift to drag ratio for the best range performance. Finally the descent profile shown in figure 3.3 shows a higher frontal profile due to the increase in twist distribution. This increases the total lift at the expense of increased drag. This corresponds to the descent performance index where more emphasis is placed on increasing lift than decreasing drag. It should be noted that the descent configuration resembles close to a gull shaped wing which a bird may perform.

As mentioned previously, the above wings are divided into 25 modules thus the parameters do not have an entirely smooth transition from one module to the next. This is particularly obvious in the cruise and descent cases. These configurations are acceptable to carry on into future steps as smoothing will be made in the next section so that parametric equations 
can be defined. See section 3.6 for discussion of reducing noise in future optimization procedures.

\subsection{Profile Smoothing}

A curve fitting tool in Matlab was used for smoothing of the initial wing configurations found in the previous section. By plotting the $1 / 4$ chord point for each module at $\mathrm{x}, \mathrm{y}, \mathrm{z}$, location as well as the twist distribution, polynomials were fitted to the geometry. These polynomials were in turn used as the parametric equations necessary for discretization (see section 4.3).

For climb the parametric equations chosen were

$$
\begin{aligned}
& s_{\text {clim }, x}[m]=0.5223 t^{2}+1.6173 t+0.25 \\
& s_{\text {clim }, y}[m]=-0.18 t^{3}+0.7414 t^{2}-0.0184 t \\
& s_{\text {clim }, z}[m]=-0.5539 t^{2}+2.7032 t \\
& s_{\text {clim }, \alpha}[\text { degrees }]=3.8274 t^{2}-3.1054 t
\end{aligned}
$$

As can be seen in the following figure 3.4, the geometry resembles closely to the initial configuration from figure 3.1 .
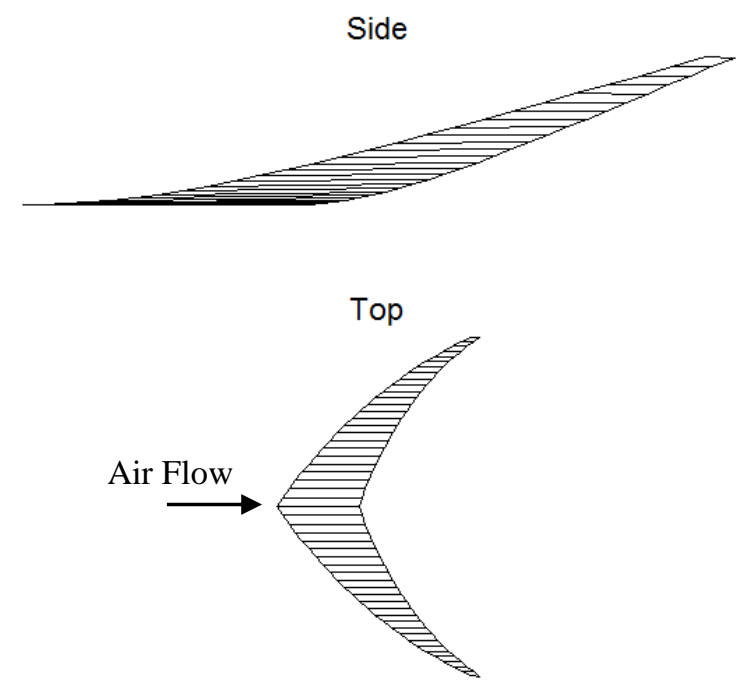

Front

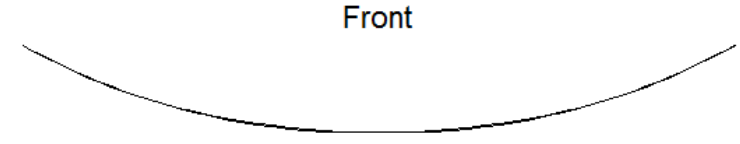

ISO

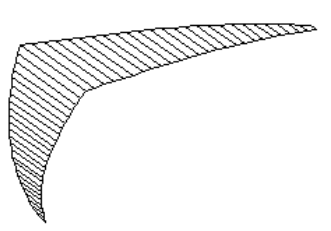

Figure 3.4: Climb Tornado Geometry

The parametric equations found for cruise were chosen to be 


$$
\begin{array}{r}
s_{\text {cruise }, x}[m]=0.3269 t^{3}+0.5113 t^{2}+0.8438 t+0.25 \\
s_{\text {cruise }, y}[m]=1.299 t^{5}-2.3697 t^{4}+1.0362 t^{3}-0.1352 t^{2}+0.0079 t \\
s_{\text {cruise }, z}[m]=-0.5827 t^{3}+0.1935 t^{2}+3.0155 t \\
s_{\text {cruise }, \alpha}[\text { degrees }]=-8.3649 t^{3}+5.9599 t^{2}+4.5092 t
\end{array}
$$

Similar to the climb case, figure 3.5 shows a similar resemblance to that of the configurations in figure 3.2. Particular smoothing occurred in the twist distribution as can be seen in the front view of figures $3.2 \& 3.5$ close to the wing root (center of front view).
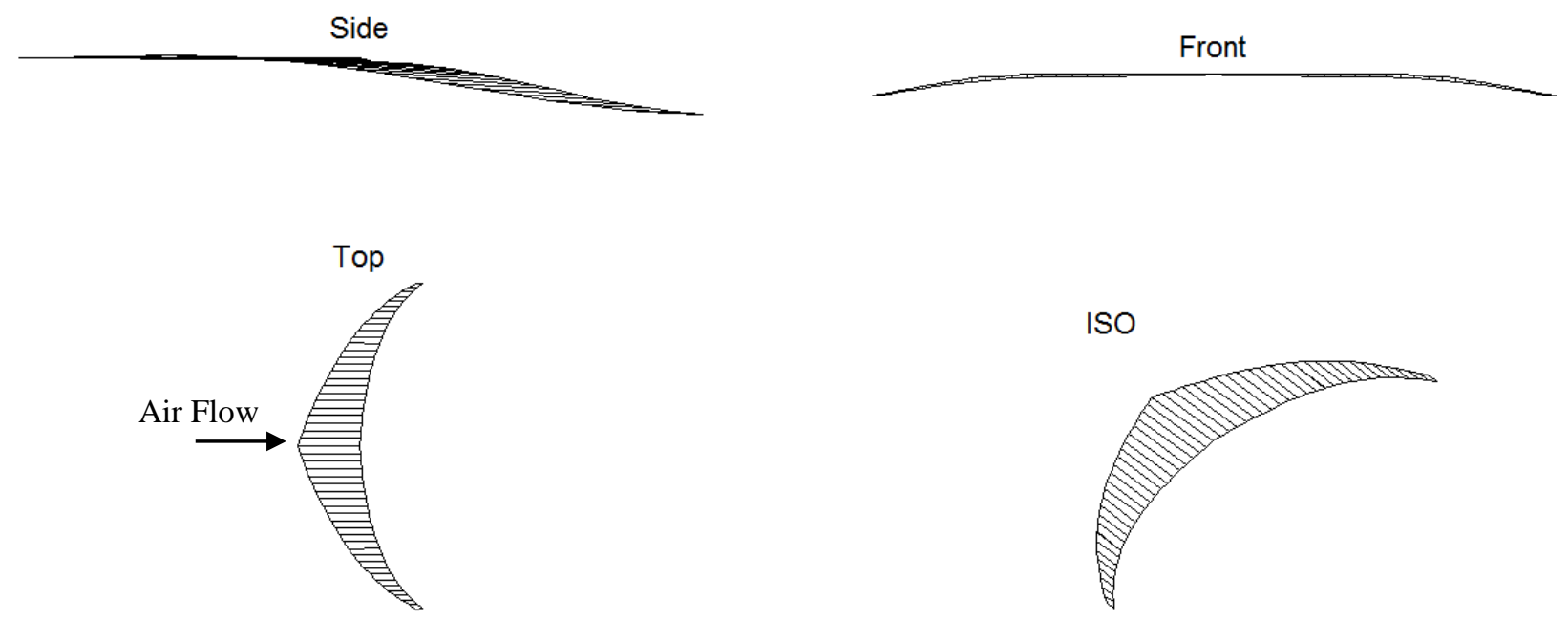

ISO

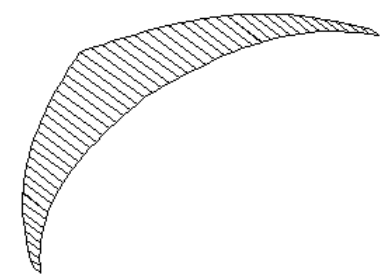

Figure 3.5: Cruise Tornado Geometry

Lastly the parametric equations found suitable for the descent configuration were

$$
\begin{gathered}
s_{\text {descent }, x}[m]=-1.5137 t^{4}+3.3238 t^{3}-2.1426 t^{2}+0.5237 t+0.25 \\
s_{\text {descent }, y}[m]=3.249 t^{5}-5.7302 t^{4}+1.5708 t^{3}+0.7068 t^{2}+0.1123 t \\
s_{\text {descent }, z}[m]=-0.0561 t^{2}+2.9923 t \\
s_{\text {descent }, \alpha}[\text { degrees }]=-8.0002 t^{4}+17.781 t^{3}-17.603 t^{2}+19.318 t
\end{gathered}
$$

Figure 3.6 shows the wing geometry generated from the above parametric equations, strong resemblance can be seen between this and the initial configurations in figure 3.3. 

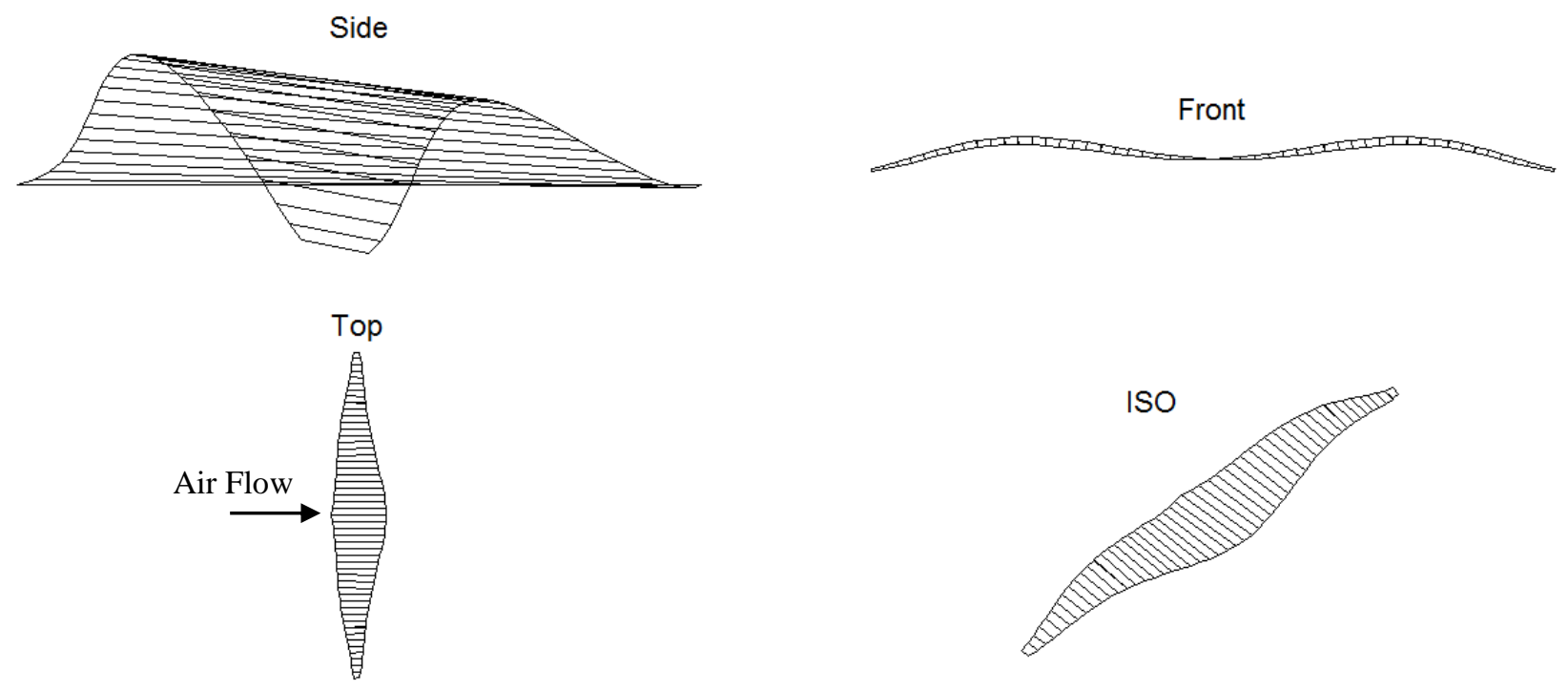

Figure 3.6: Descent Tornado Geometry

Smoothing along the leading edge in the side view of figures $3.3 \& 3.6$ can be seen.

The parametric equations listed here were deemed satisfactory as the geometries strongly resemble those of the initial conditions. The wings shown in this section, figures 3.4 through 3.6, represent the reference wings needed for discretization to occur.

\subsection{Performance Check}

Since smoothing of the initial wing configurations was performed, the performance parameters much be checked so that no major reduction in performance occurred.

Table 3.3: Wing Profile Performance Check

\begin{tabular}{|l|l|l|l|}
\hline & Climb & Cruise & Descent \\
\hline Initial & $\mathrm{C}_{\mathrm{L}}: 0.202$ & $\mathrm{C}_{\mathrm{L}}: 0.749$ & $\mathrm{C}_{\mathrm{L}}: 1.11$ \\
& $\mathrm{C}_{\mathrm{D}}: 0.0116$ & $\mathrm{C}_{\mathrm{D}}: 0.0259$ & $\mathrm{C}_{\mathrm{D}}: 0.0356$ \\
\hline Smooth & $\mathrm{C}_{\mathrm{L}}: 0.210$ & $\mathrm{C}_{\mathrm{L}}: 0.734$ & $\mathrm{C}_{\mathrm{L}}: 0.98$ \\
& $\mathrm{C}_{\mathrm{D}}: 0.0108$ & $\mathrm{C}_{\mathrm{D}}: 0.0268$ & $\mathrm{C}_{\mathrm{D}}: 0.0412$ \\
\hline CFX Smooth & $\mathrm{C}_{\mathrm{L}}: 0.198$ & $\mathrm{C}_{\mathrm{L}}: 0.665$ & $\mathrm{C}_{\mathrm{L}}: 0.933$ \\
& $\mathrm{C}_{\mathrm{D}}: 0.0112$ & $\mathrm{C}_{\mathrm{D}}: 0.0308$ & $\mathrm{C}_{\mathrm{D}}: 0.0443$ \\
\hline
\end{tabular}

As can be seen the error is relatively low where the largest change occurred with descent where $C_{L}$ and $C_{D}$ had an error of $13.3 \%$ and $15.7 \%$, respectively. This could be due to analysis error caused by the smooth of the twist distribution. This could likely be due to analysis error due to the noisy initial descent configuration. For climb and cruise the error is about $5 \%$. This falls 
under acceptable values as each case still outperforms the others at their respective performance index. See Appendix for delta $\mathrm{C}_{\mathrm{P}}$ plots for each flight regime.

The CFX results for the identical smooth wing was also shown in the above table for the sake of comparison. Both geometries from the 'smooth' and the 'CFX smooth' are identical and a grid independent study was also performed (see section 4.1.2) so any difference in results can be was due to the solver program. The errors experienced here are slightly larger than those of the initial and smooth cases. Although the errors are within acceptable range (5-10\%), it's an important note that although smoothing would cause a difference in performance, the vortex lattice method has its own set of errors which need to be checked with a higher accuracy model such as Ansys CFX (see chapters $4 \& 5$ ).

\subsection{Parameter Noise}

Generally simplicity in design is preferred for engineering applications. Although there are many complicated aspects of a modular morphing wing, avoiding further unnecessary complexity is important. Finding aerodynamic configurations is no stranger to this. Avoiding abrupt changings in geometry throughout the wing can help avoid adverse effects such as flow separation leading to stall, and unstable conditions when transforming from one configuration to another.

Although the approximation was straight forward for most of the parametric curves, there were a few instances that had to be simplified such as the twist distribution for cruise. One method of solving this issue would be to strictly measure the goodness of fit. There are a number of tests that can be used but this does not exactly solve the problem of complex geometries. The parameter noise would be slightly dampened when converting the initial wing to a smooth one but it would nonetheless still be present.

A more suitable approach would be to change the optimization procedure. The harmony search algorithm can still be used but the initial search would use few modules instead of 25 . Also the parameter limits should have a higher constraint for the initial runs. This would successfully narrow the design when starting the initial search. Once a suitable wing is found for a specific flight regime, the user would check if any of the limits are consistently being hit. An 
example would be if the sweep angle constantly hits a limit set to $10^{\circ}$, this suggests the potential for greater performance index values if the limit was increased. Once limits are established, further optimization can be done with increasing the number of modules. An example would be that starting with 4 modules then increasing gradually toward 10 . Wings with more than 10 modules can be generated but, as will be seen in section 4.4, this may be redundant as the performance index increase per added module would plateau.

This method gives the user vital feedback into what parameters are more important than others as each step gradually increases the design space. One particular flight regime might show more emphasis on changing the twist parameters while another may benefit more by increasing the cant distribution. Since this is a repetitive process the parameters can be more closely monitored to avoid parameter noise without sacrificing performance potential by otherwise arbitrarily constraining parameters. This feedback would also allow the user to increase modules in areas that are believed to be important before repeating the optimization procedure. Although there is user guidance involved in this type of optimization routine, by reviewing each step before expanding the design space there is more room to explore new areas. For the purposes of this thesis report however the initial wing configurations found were suitable to continue with discretization and consolidation procedures. 


\section{Discretization Profile}

The previous chapter found reference wings using an optimization routine in conjunction with a vortex lattice method solver. These reference wings were found separately by optimizing for performance objectives based on climb, cruise, and descent flight regimes.

This chapter continues with discretizing the reference wings into linear segments to make modular wings. The objective for this chapter is to create modular wings for each flight regime with the minimal number of modules without significantly sacrificing performance.

Figure 4.1, developed by Finistauri [19], governs the steps involved for the discretization loop.

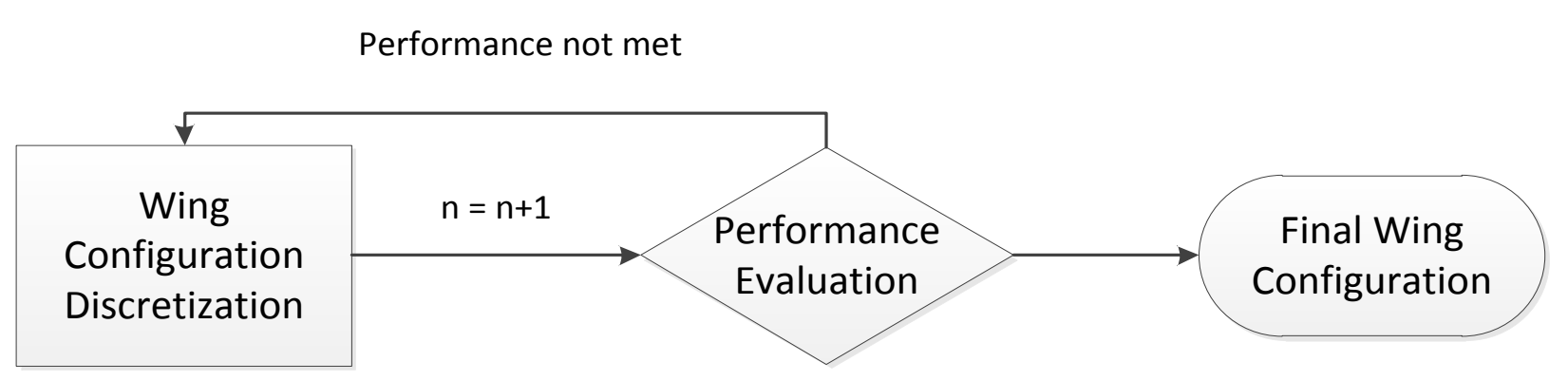

Figure 4.1: Discretization Loop [19]

The flow chart is suitable for one flight objective thus this step is carried out for each flight regime in parallel. The wing configuration discretization involves converting the reference wings, from chapter 3 , into modular wings composed of linear segments. These discretized 
wings are then evaluated, in respective to their performance index, for varying number of modules (represented by $\mathrm{n}=\mathrm{n}+1$ in figure 4.1 where $\mathrm{n}=$ number of modules). For this case wings with modules 2 to 10 were considered. As will be seen in section 4.4, no more than 10 modules were needed to receive noticeable performance increase.

\subsection{Computational Methods and Conditions}

\subsubsection{Computational Fluid Solver}

Unlike the previous wing profile chapter, a finite element method CFD code called Ansys CFX was used chosen for discretization and consolidation analysis due to the high degree of accuracy relative to other software packages. The cost is however in the runtime as a typical runtime would be in excess of 24 hours running on 8 computer cores. This time would be impractical for the optimization routine but is acceptable for discretization and consolidation purposes.

The flow conditions were set to standard sea level at 0.3 Mach with a Reynolds number of 4683000 with a $3^{\circ}$ angle of attack. It was assumed the flow was viscous, incompressible, and isothermal. The turbulence model chosen was the shear stress transport (SST) model as it can accurately analysis the boundary layer. This is particularly important for the joints between modules where boundary layer interaction is expected. The wing was set to a no slip wall condition. The following figure shows the fluid domain used. 


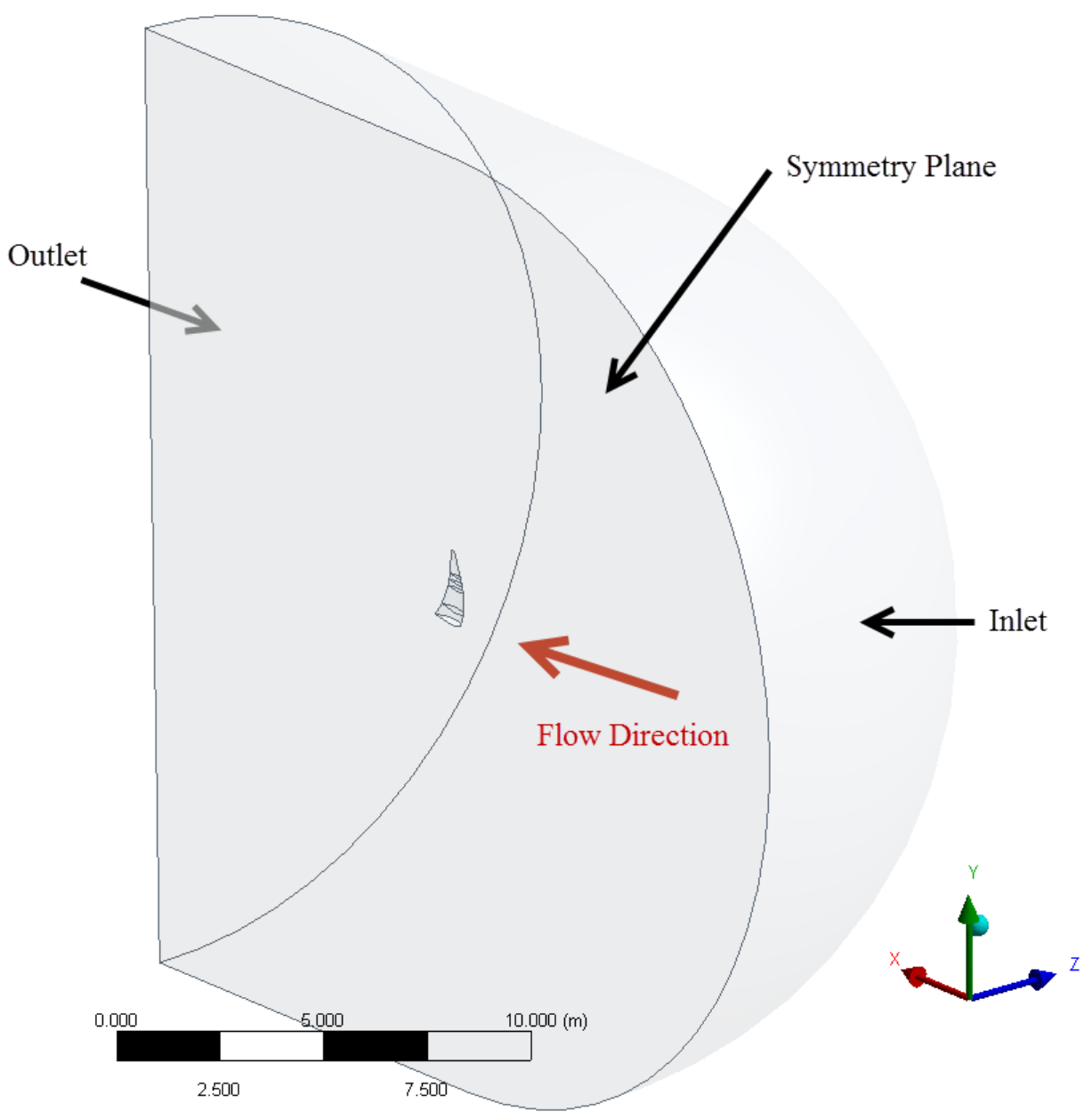

Figure 4.2: CFX Fluid Domain

As can be seen a symmetry plane was placed along the wing root. The fluid domain extents $12 \mathrm{~m}$ outward from the wing toward the inlet and outlet. The fluid flow is primarily along the $\mathrm{x}$-axis, where small velocity component in the y-axis due to the angle of attack.

\subsubsection{Computational Grid}

Since CFX was used for this section of the analysis the Ansys Workbench Mesher was chosen for the grid generation. The 3D grid is composed of inflation layers (prism shaped elements) built from the wing surface and the remaining space filled with tetrahedral shaped elements. A grid independent study was performed for the climb configuration which was then used for grid settings for each case. Adjustments were made on a case by case basis as face sizing was slightly changed as well as refinement and smoothing when necessary depending on grid quality. 
The general grid settings was an initial prism layer of thickness of $3.2 * 10^{-6} \mathrm{~m}$ which allowed for a Y+ order of 1 as needed for the shear stress transport (SST) turbulence model. The amount of prism layers was set to 40 with a growth rate of 1.21. The wing surface face sizing was generally between $8 * 10^{-3} \mathrm{~m}$ and $4 * 10^{-3} \mathrm{~m}$ from root to tip. This specific value would fluctuate slightly depending on the mesh quality.

Figures 4.3 through 4.5 show the Ansys grid. Figure 4.3 shows a view of the symmetry and outlet region of the fluid domain where the red dashed rectangle leads to the outline of figure 4.4. Figure 4.4 gives a view of the grid surrounding the wing root which displays the relative total prism layer thickness as well as transition to tetrahedral shaped elements. The dashed inner rectangle in figure 4.4 represents the outline for figure 4.5 where a zoomed in view of the prism layers is shown.

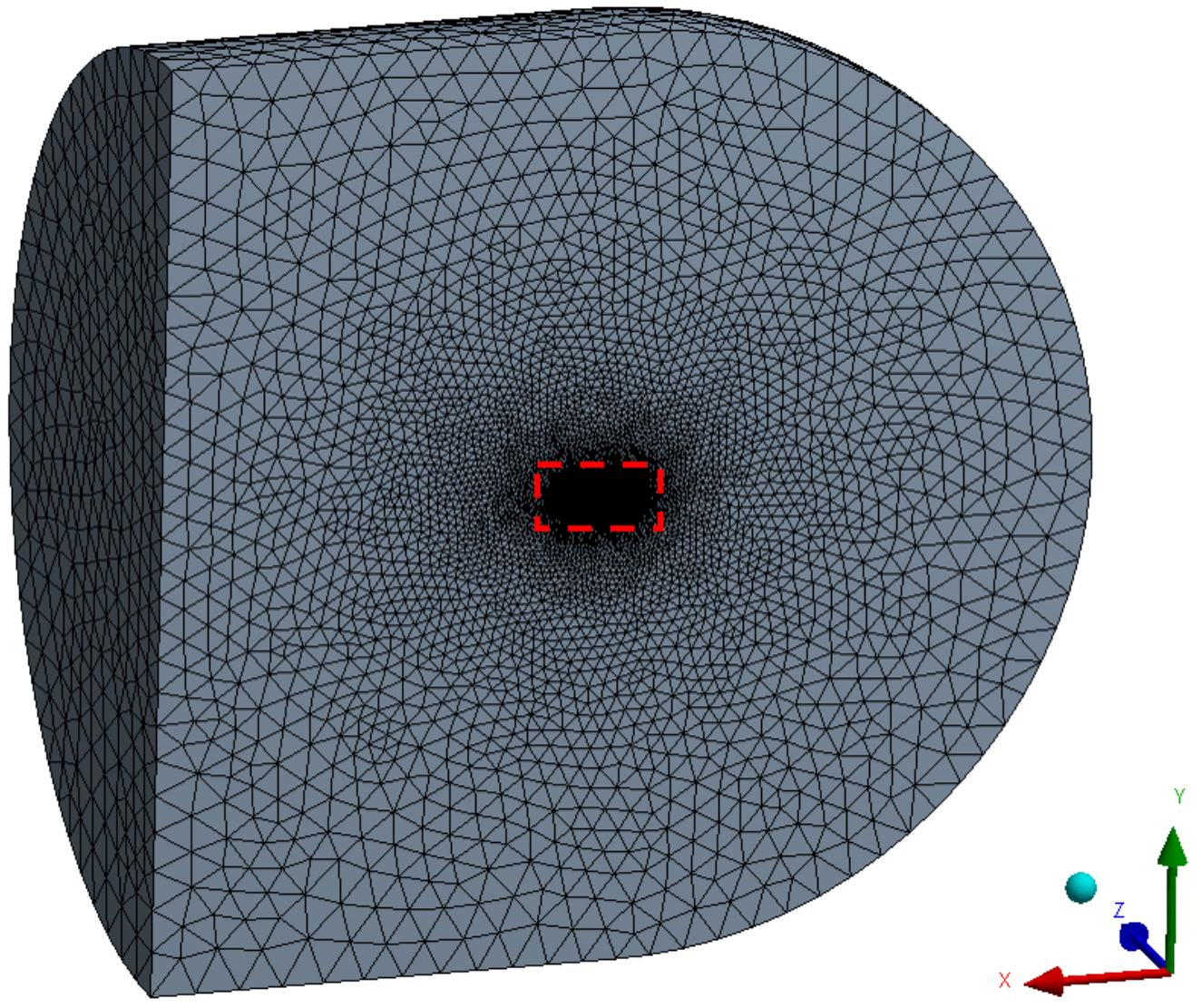

Figure 4.3: Ansys Grid Fluid Region 


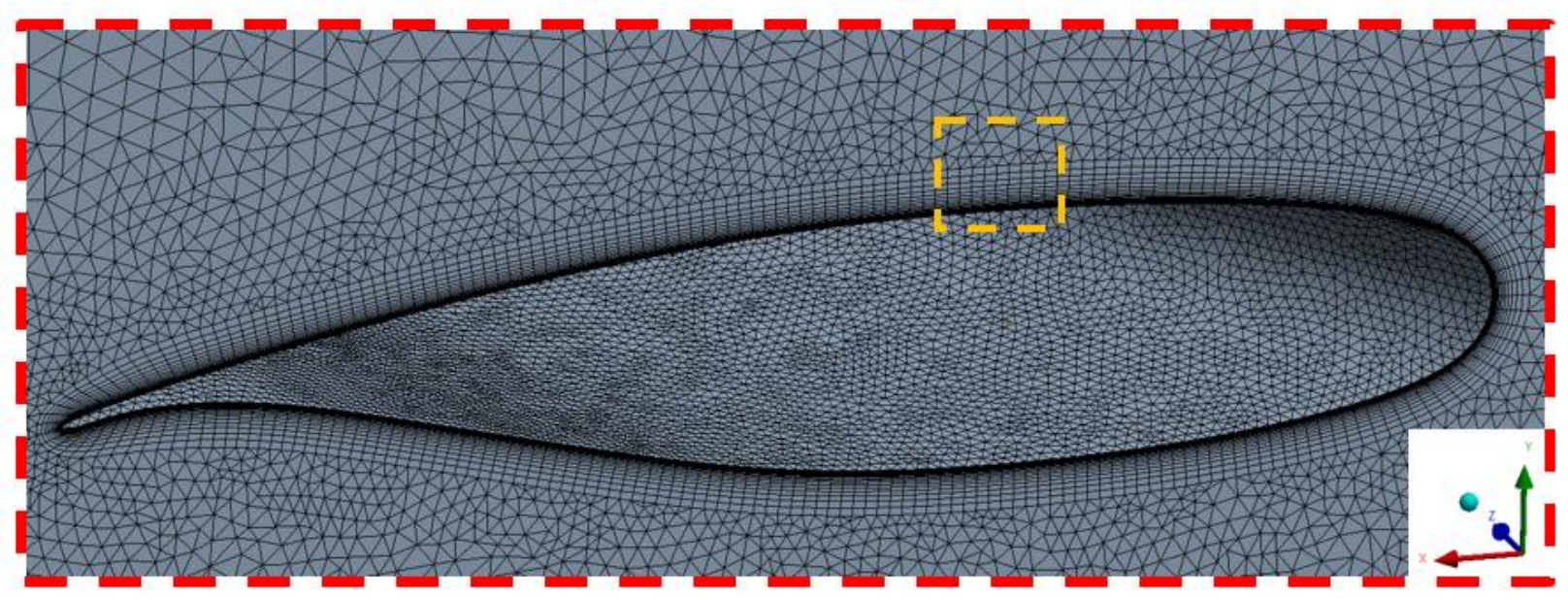

Figure 4.4: Ansys Grid Wing Cross Section
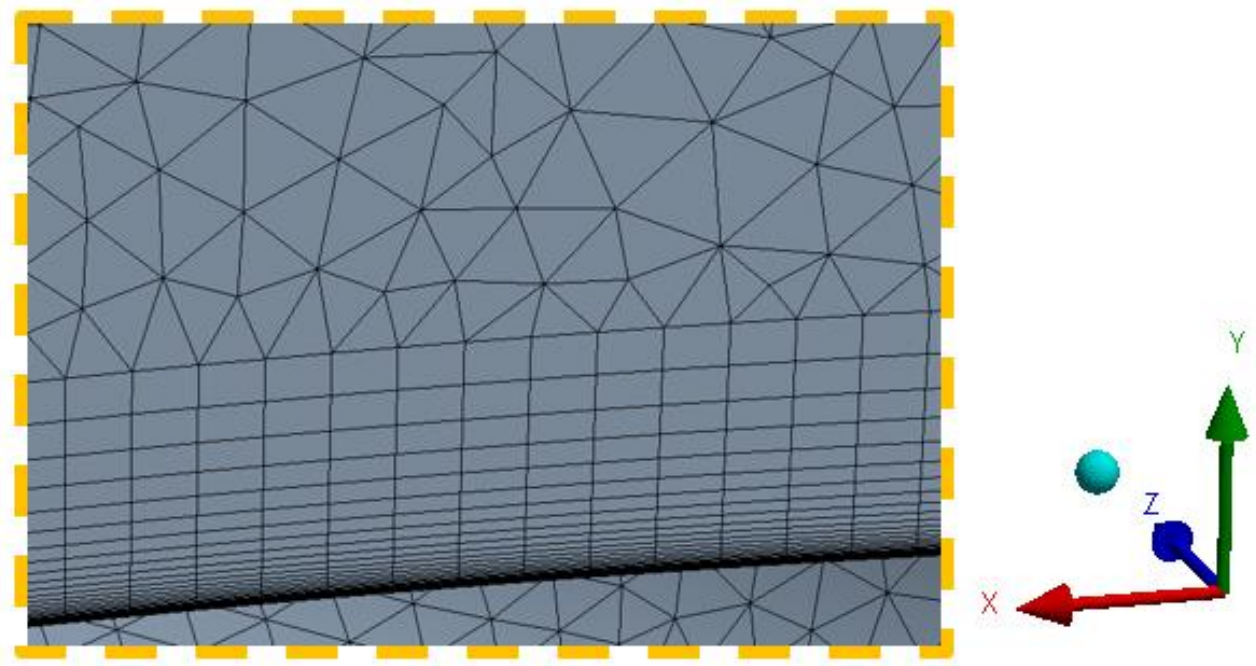

Figure 4.5: Ansys Grid Prism Layers

The following figure shows the grid independent study where the general wing surface face sizing, prism growth rate, and number of prism layers was determined. 


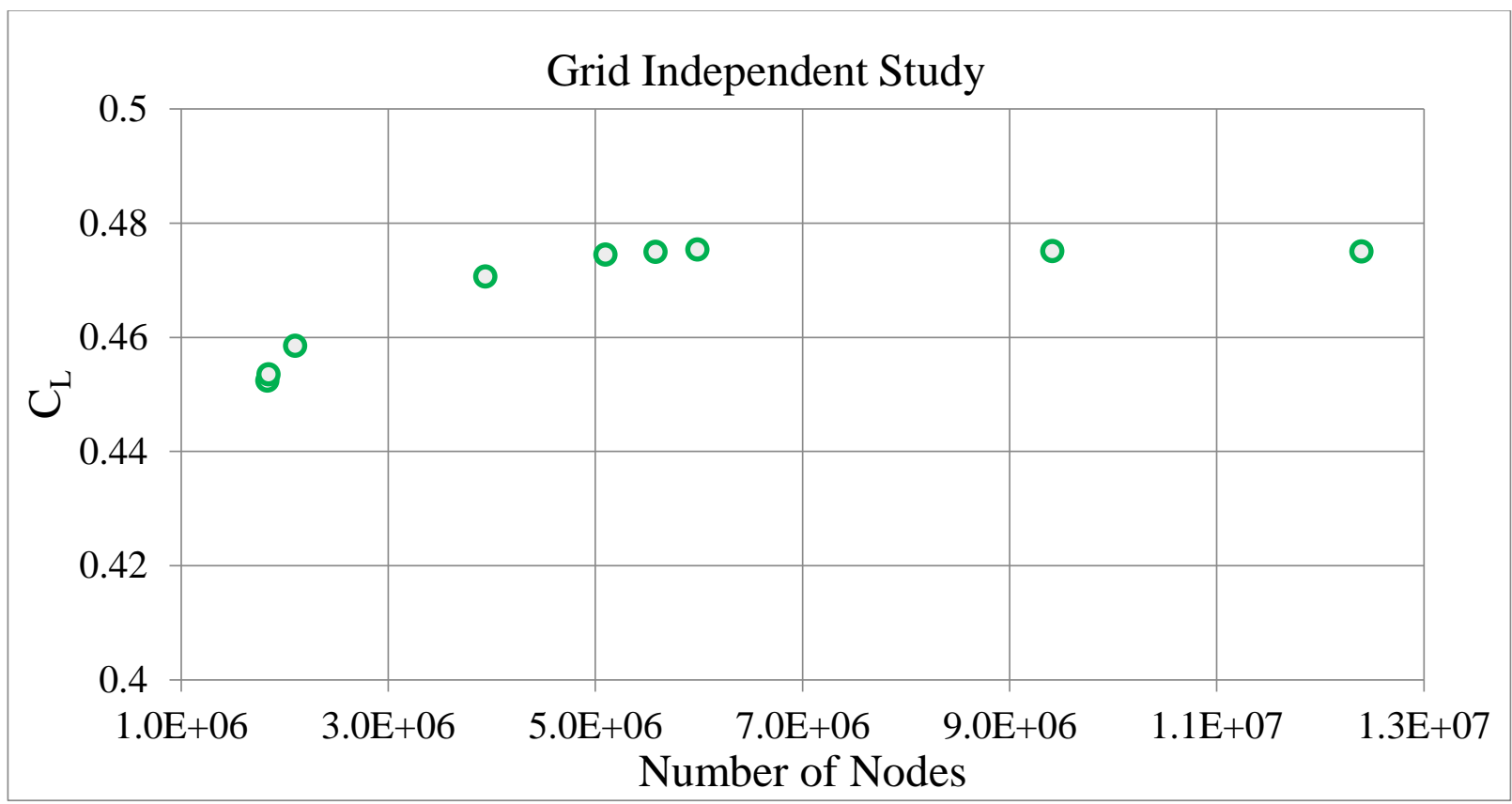

Figure 4.6: Ansys Grid Independent Study

As can be seen greater than approximately 5 million nodes does not have any further gain in $\mathrm{C}_{\mathrm{L}}$ values. The actual change in values was within $0.9 \%$. Thus the generated grids for each simulation, with the mentioned grid sizing, had approximately 6 million nodes. It is important to note that experimental work which resembled a wing made of linear segments was not available thus this grid independent study was used to valid the grid. Changes in performance greater than $0.9 \%$ can then be accredited to changes within the actual physics and not dependent on the model setup.

\subsection{Discretization Methodology}

This section explains the basic steps involved for geometry discretization outlined by Finistauri et al. [4] based on a reference wing configuration. This approach was chosen so that modules are spaced according to the reference wing geometry and not simply evenly spaced from root to tip. Areas of the reference wing with high curvature, such as the joining curve of a wingtip and the main wing body, receive shorter modules while other sections with little curvature receive longer modules. This unique feature allows for the minimizing of the number of modules needed to represent the wing without greatly sacrificing the general shape. 
The first step involved defining the reference wing into parametric equations. These equations represent the wing geometry in the $\mathrm{x}, \mathrm{y}, \mathrm{z}$ directions as well as the twist distribution.

$$
s(t)=\left\{s_{x}(t), s_{y}(t), s_{z}(t), s_{\alpha}(t)\right\}^{T}
$$

Where $s$ represents the space curve for each component $\mathrm{x}, \mathrm{y}, \mathrm{z}$, and $\alpha$ (twist). $t$ is the wing arc length, or called the line space from root to the tip of the wing. It is important to note that these equations are arc-length parameterized in which is represented by $t$. the arc-length range is $0 \leq t \leq 1$ where 0 and 1 represent the root and tip, respectively. The first three parameterized components of equation (4.1), $s_{x}, s_{y}, s_{z}$, represent the $3 \mathrm{D}$ position of the $1 / 4$ chord point along the wing from root to tip ( $t=0$ to $t=1)$. The twist distribution would then be represented by $s_{\alpha}$, which is about the $1 / 4$ chord line from root to tip.

Allowing $s_{X}(t)=\left\{s_{x}(t), s_{y}(t), s_{z}(t)\right\}^{T}$, the instantaneous curvature at any point $t$ is calculated using Frenet-Serret formula and shown by equation (4.2).

$$
\kappa(t)=\frac{\left\|\dot{s}_{X}(t) * \ddot{S}_{X}(t)\right\|}{\left\|s_{X}(t)\right\|^{3}}
$$

Where $\kappa$ represents the curve curvature. The twist distribution is defined by torsion in equation (4.3).

$$
\tau(t)=\frac{\left|\dot{s}_{\alpha}(t)\right|}{\left\|s_{X}(t)\right\|}
$$

Where $\tau$ is the torsion. Using equations (4.2) \& (4.3), the total curvature can be calculated using equation (4.4).

$$
X=\int_{0}^{1} \sqrt{(\kappa(t))^{2}+(\tau(t))^{2}}
$$

Where $X$ is the total wing curvature. With equation (4.4), the total curvature is known for a reference wing. Equation (4.5) calculates the average curvature each module would possess by dividing the total curvature by the number of desired modules. This allows the modules to be spaced according to the reference wing's curvature.

$$
\bar{X}=X / m
$$


Where $\bar{X}$ is the average wing curvature, and $m$ is the number of modules. Solving for $t$ numerically would determine the nodal spacing which would be formed as,

$$
T=\left\{0, t_{1}, t_{2}, \ldots, t_{k} \ldots, t_{m-1}, 1\right\}
$$

Where $T$ is the nodal spacing array. Note that in equation (4.6), 0 and 1 are always present as these are the bounds of the curvature at the root and tip. A wing with 2 modules would then be noted by $T=\left\{0, t_{1}, 1\right\}$ and 4 modules as $T=\left\{0, t_{1}, t_{2} . t_{3}, 1\right\}$.

Further explanation of this discretization method as well as a case study using Fluent CFD solver is outlined by Finistauri et al. [4].

\subsection{Discretize Wing Profile}

Since the morphing procedure chosen was that of a modular wing, the smooth wing geometries created in the previous section must be divided into linear sections. Using the procedure outlined in the previous section, a Matlab code (written by Finistauri et al. [4]) was used to discretize the reference geometries for climb, cruise and descent flight regimes into 2 to 10 modules. It should be noted that a single module wing was not considered as it would have a drastically different performance from that of the reference wings thus the results would be misleading. As Ansys Workbench is used here for analysis, the wing geometry was recreated into a CAD file via an ICEM CFD script developed by Paudel [24].

As expected the joint locations were placed in areas that contains high amount of wing line curvature. As the number of modules are increased, clusters of joints form in these areas. For illustration purposes, joints for wings containing 2, 4, 6, and 10 modules are shown in the figures below. The following figures shows the geometries in Matlab as the flat plat allows the wing parameters, sweep, cant, twist, and span to be easily viewable. In reality the CAD geometries use the super critical airfoil sc20518. The full list joint locations for wings with modules 2 through 10 can be found in the appendix as well as geometries values for each module for the respective figures below. 


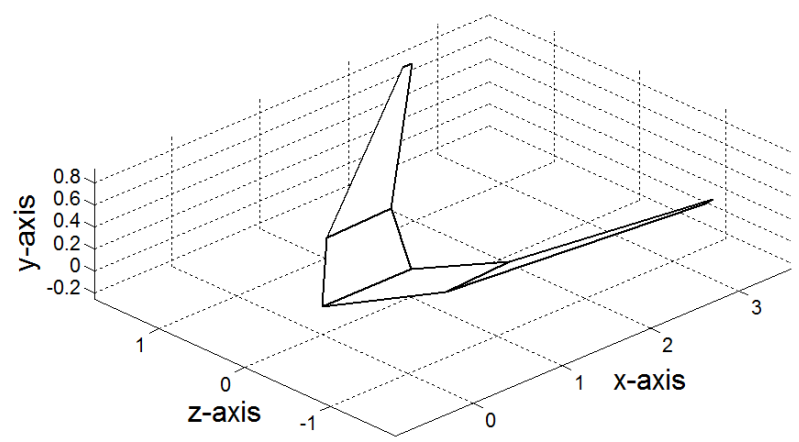

Figure 4.7: Climb 2 Modules

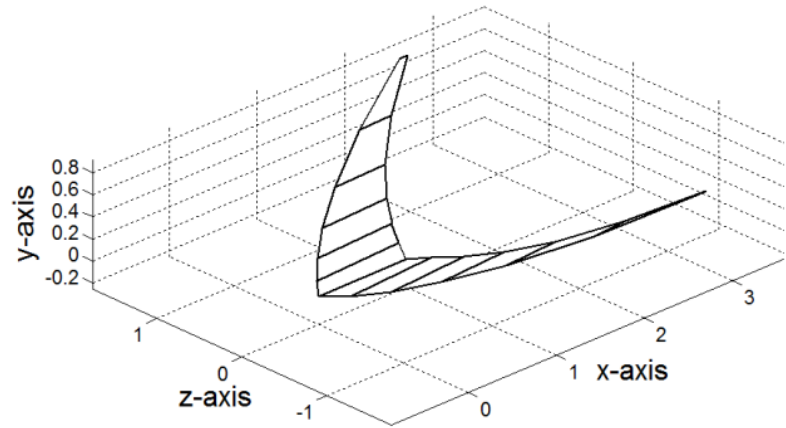

Figure 4.9: Climb 6 Modules

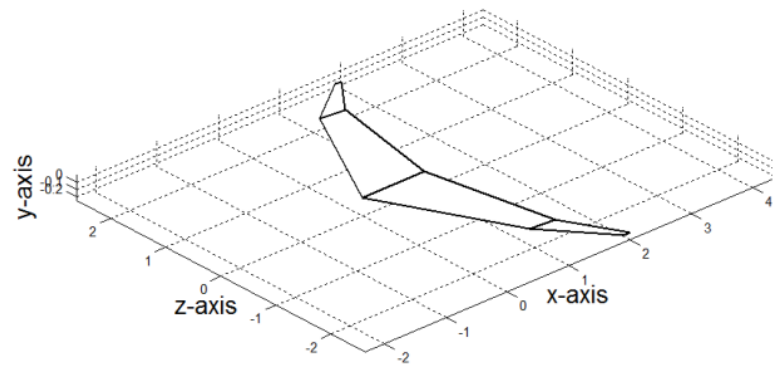

Figure 4.11: Cruise 2 Modules

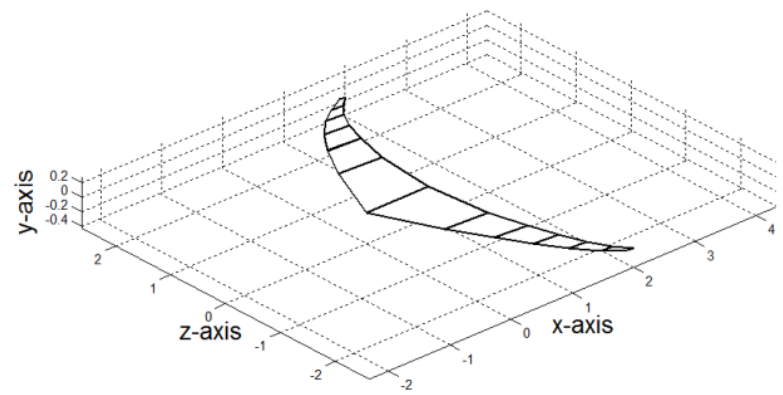

Figure 4.13: Cruise 6 Modules

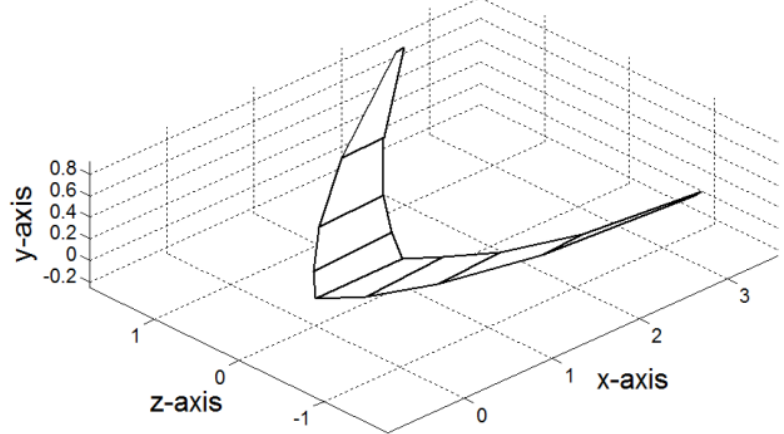

Figure 4.8: Climb 4 Modules

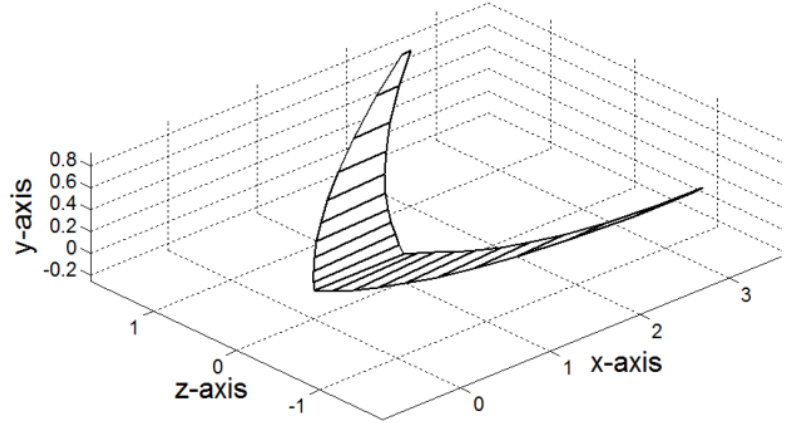

Figure 4.10: Climb 10 Modules

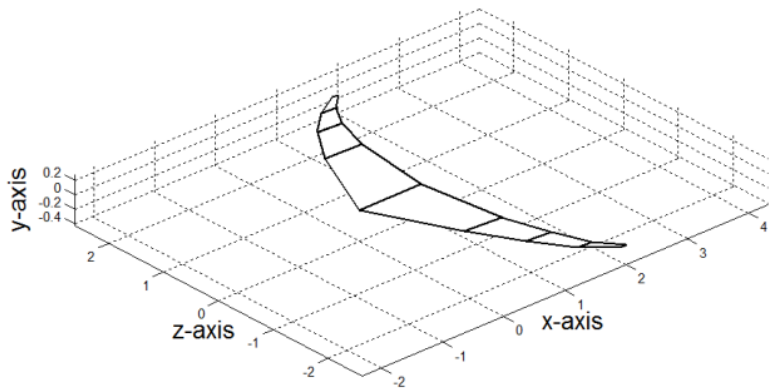

Figure 4.12: Cruise 4 Modules

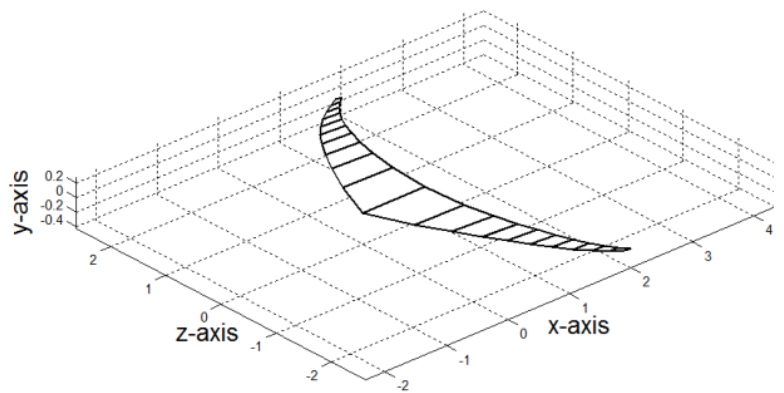

Figure 4.14: Cruise 10 Modules 


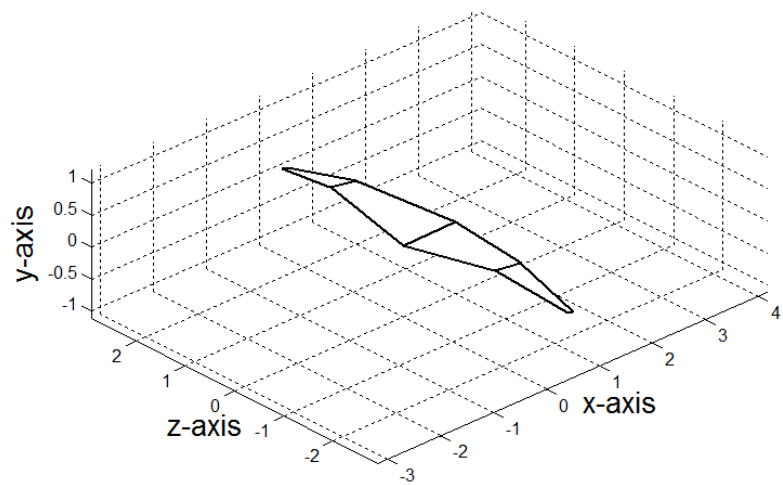

Figure 4.15: Descent 2 Modules

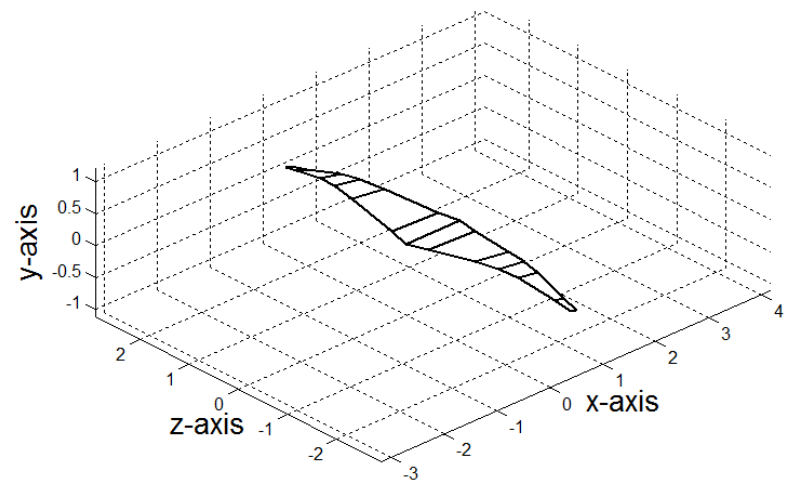

Figure 4.17: Descent 6 Modules

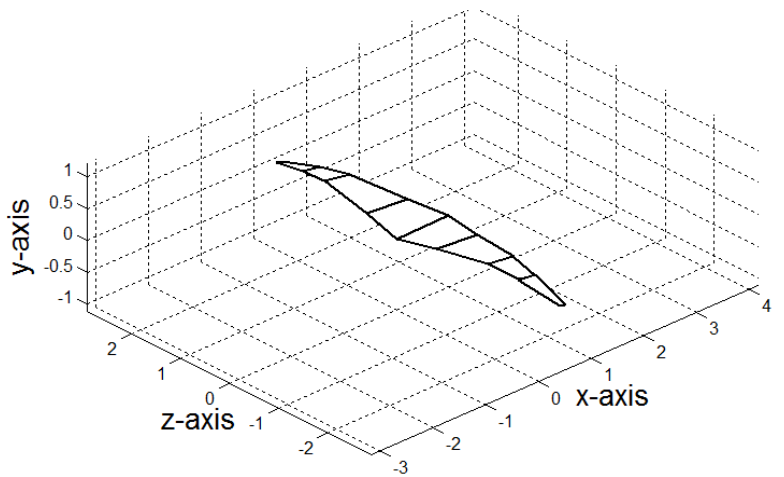

Figure 4.16: Descent 4 Modules

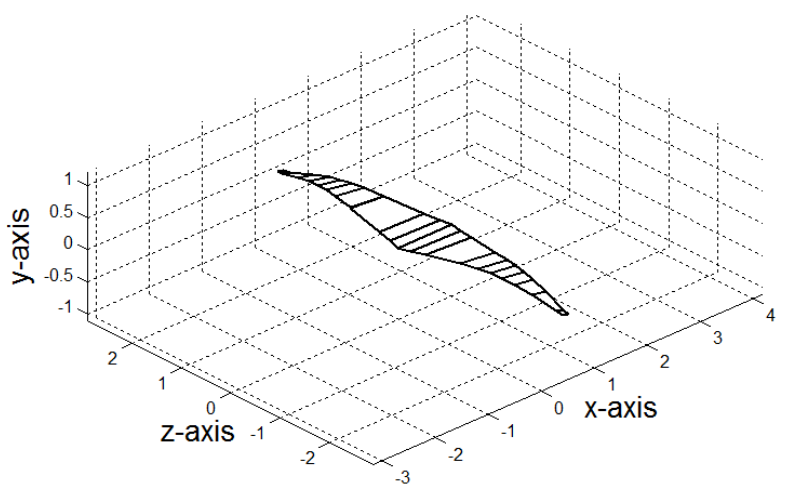

Figure 4.18: Descent 10 Modules

Notice the climb configurations (figures 4.7 through 4.1) are divided nearly evenly along the arc length where a slight concentrate is close to the root. This is because the sweep, dihedral, twist, and span distributions are relatively continuous from root to tip without any major changes.

The cruise and descent configurations however show more defined areas with larger change in parameter. Both cruise and descent show joint concentration closer to the tip of the wing. Descent shows signs of joint concentration closer to the root especially when looking at figure 4.18 for 10 modules.

The wing geometries needed for the block, 'wing configuration discretization', of figure 4.1 is now created. 


\subsection{Minimum Modules}

The number of sections present in a discretized wing has a large impact in the complexity of the system. Therefore a practical approach to using a modular wing is to reduce the number of modules without significantly sacrificing that wing's performance.

The process illustrates the repetitive loop shown in figure 4.1 so that discretized wings are evaluated until stopping criteria where performance gain is no longer observable. Each set of discretized wings created in the previous section were analyzed in CFX using the boundary condition and grid generation mentioned in sections 4.1.1 and 4.1.2, respectively. Windows batch, shell, and python scripts were created to automate this process. For each flight regime, the minimum number of modules was chosen to represent the wing based on the performance index values.

\subsubsection{Climb Module Analysis}

As previously discussed, the performance index for climb is to minimize $C_{D}$ (see section 3.2). Although both $\mathrm{C}_{\mathrm{L}}$ and $\mathrm{C}_{\mathrm{D}}$, along with $\mathrm{CFX}$-solver residuals, were monitor to determine if the simulation converged or not, $\mathrm{C}_{\mathrm{D}}$ values had the greatest importance for the climb flight regime. 


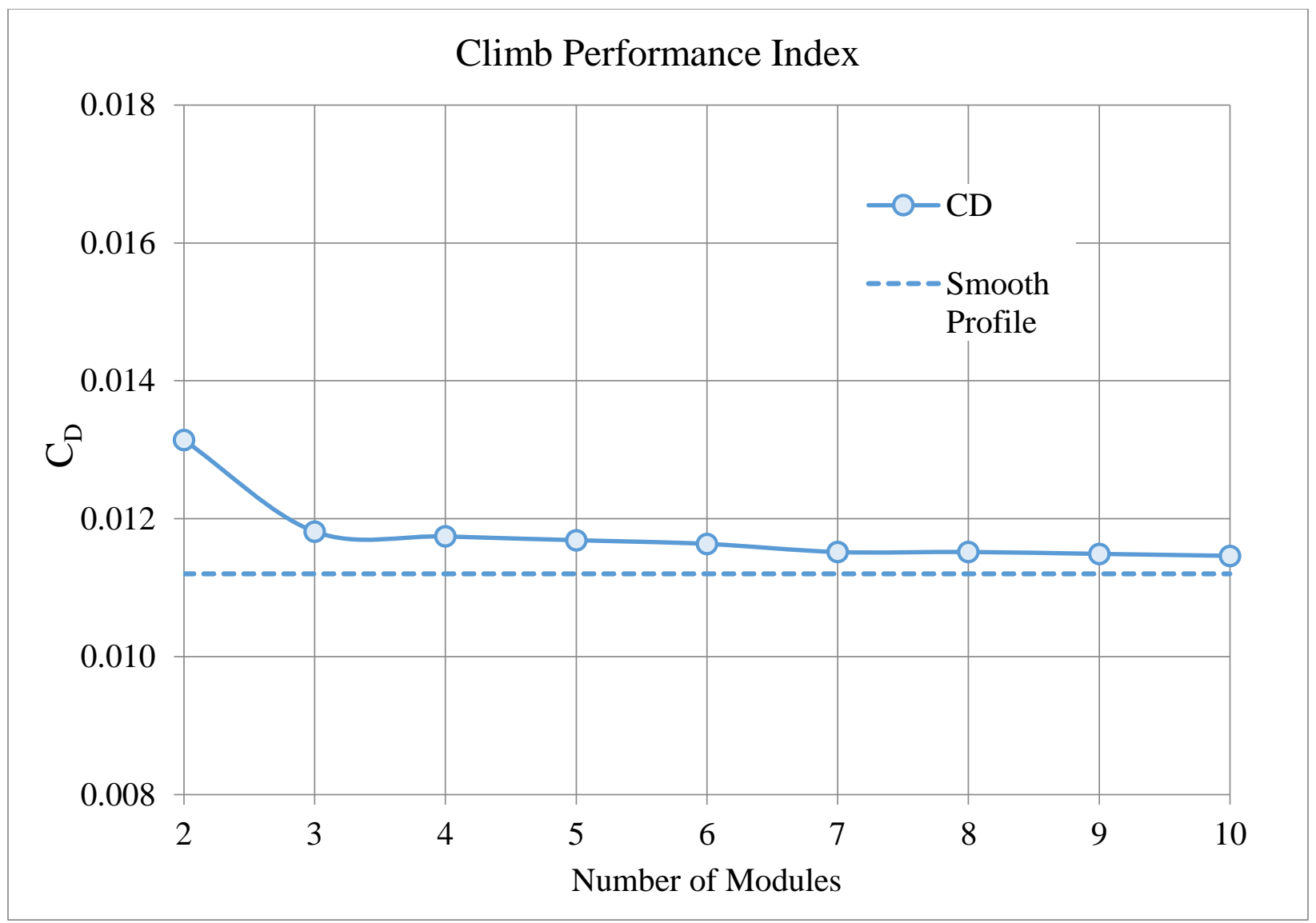

Figure 4.19: Climb Discretization Performance Index

Figure 4.19 shows the $C_{D}$ value for discretized wings with 2 to 10 modules, represented by the circular points. The dashed line represents the reference wing which was developed purely from the parametric equations thus is not comprised of linear segments. Since this case the objective is to minimize drag, the lower the value equates to better performance. As can be seen, there is a significant increase in performance from 2 to 3 modules before plateauing, especially when increasing the number of modules from 7 . Thus the discretized wing with 3 modules was chosen for this case.

\subsubsection{Cruise Module Analysis}

The performance index for cruise is more traditional as it is to maximize the $C_{L}$ to $C_{D}$ ratio. Figure 4.20 displays the cruise performance based on varying the discretized number of modules from 2 to 10 . 


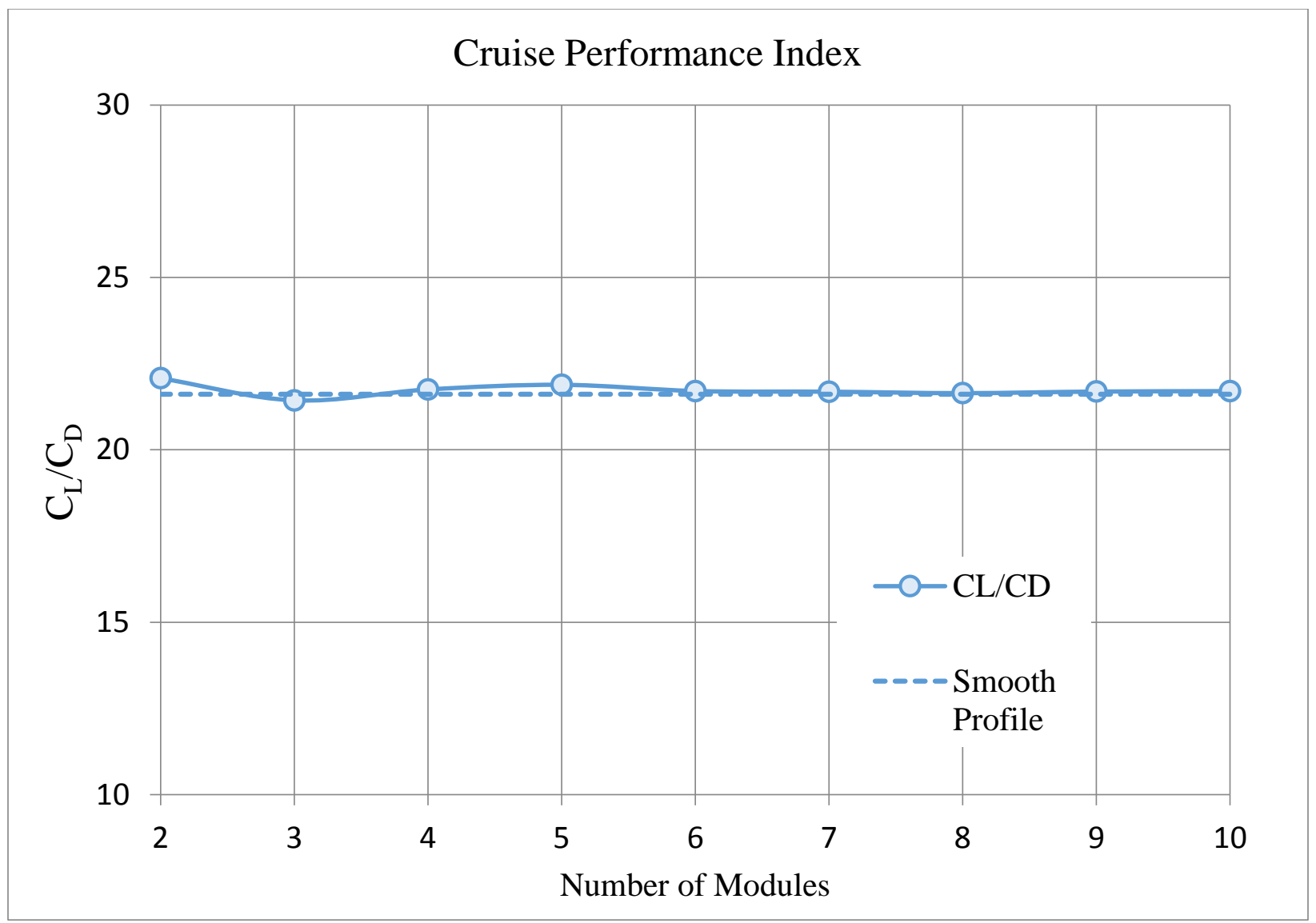

Figure 4.20: Cruise Discretization Performance Index

The diagram symbols are similar to that of figure 4.19 where the circular points represent the performance at varying number of modules. The dashed line represents the reference wing defined by the parametric equations (see section 3.4). Although the performance at 2 modules yields the best performance but the overall $C_{L} / C_{D}$ ratio is fairly constant. Although small performance variance occurs up until 6 modules, the performance afterwards converges to that of nearly the reference wing. Thus the 2 module wing was chosen for this case.

\subsubsection{Descent Module Analysis}

Finally the performance index used for descent is maximizing the $C_{L}{ }^{1.5} / C_{D}$ ratio shown in figure 4.21. 


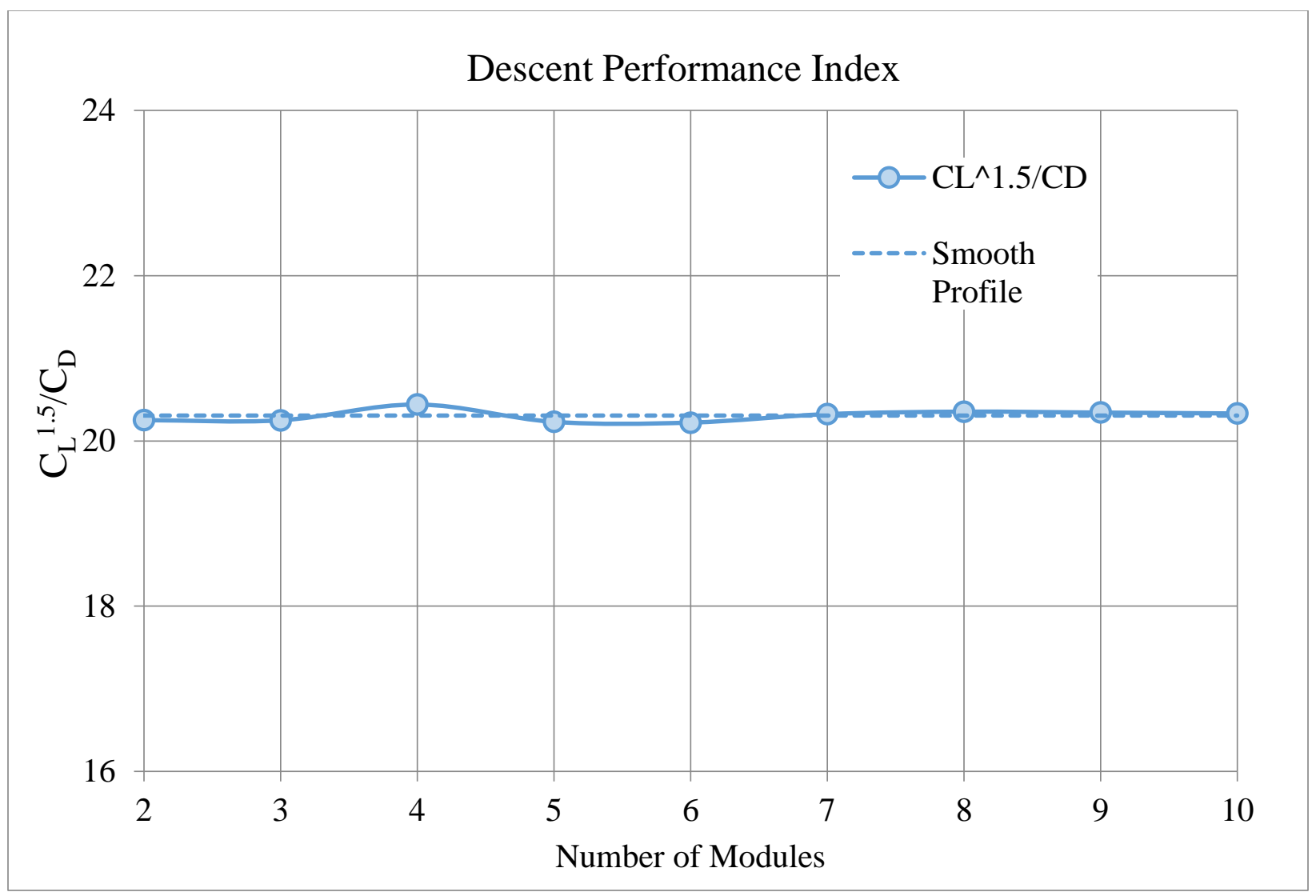

Figure 4.21: Descent Discretization Performance Index

A similar pattern can be seen from the cruise condition where the performance index does not change significantly from modules 2 through 10 . The performance index also converges to that of the reference wing when using 7 or more modules. Thus 2 modules was chosen to be suitable to represent the descent case.

\subsection{Wing Performance Discussion}

The previous section shows the performance indices for each of the three flight regimes but it is also important to consider the $C_{L} \& C_{D}$ values separately as the number of modules are increased. The following figure shows the results from the climb flight regime for modules 2 to 10 as well as the reference wing results. 


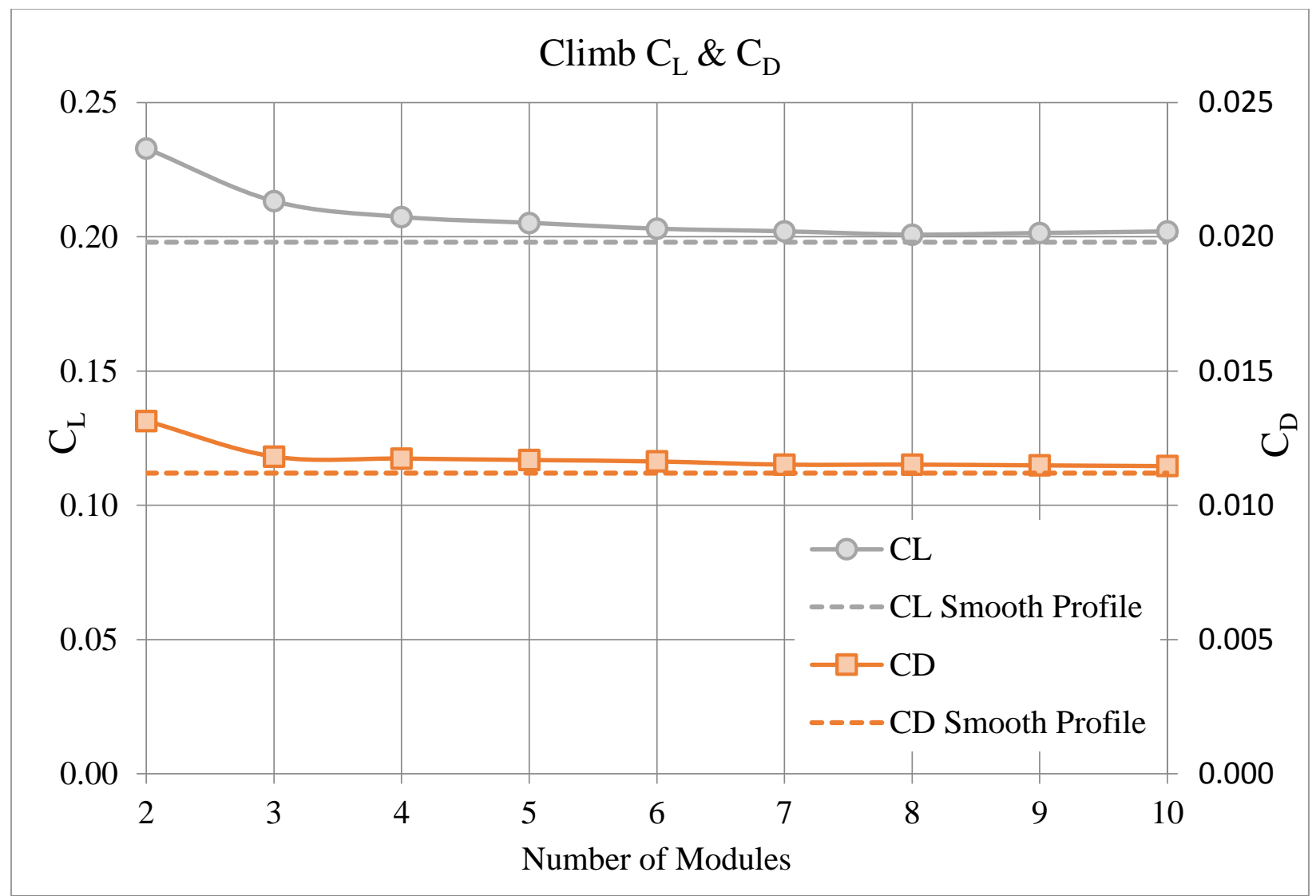

Figure 4.22: Climb Discretization $\mathrm{C}_{\mathrm{L}} \& \mathrm{C}_{\mathrm{D}}$

The left column represents the $C_{L}$ values where the circular data points are the $C_{L}$ values with respect to the number of modules. The right column represents the $C_{D}$ values where the square data points are the $C_{D}$ values. The dashed lines represent the smooth reference wing case. Figure 4.19 , from the previous section, shows the $C_{D}$ values converge toward the smooth wing case. Figure 4.22 shows the same trend with $C_{L}$ where values are lowered until it reaches approximately that of the smooth profile. As mentioned in section 3.2 the climb case performance index is to minimize $C_{D}$, thus the reduction in $C_{L}$ does not result in a reduction in the performance index. It should be noted that an analysis for a specific aircraft should include a minimum $C_{L}$ value needed to perform the climb maneuver. Since this thesis does not focus on any particular aircraft this was neglected so that unnecessary assumption were not made.

The following figure shows the $C_{L} \& C_{D}$ values for the discretized cruise configurations. 


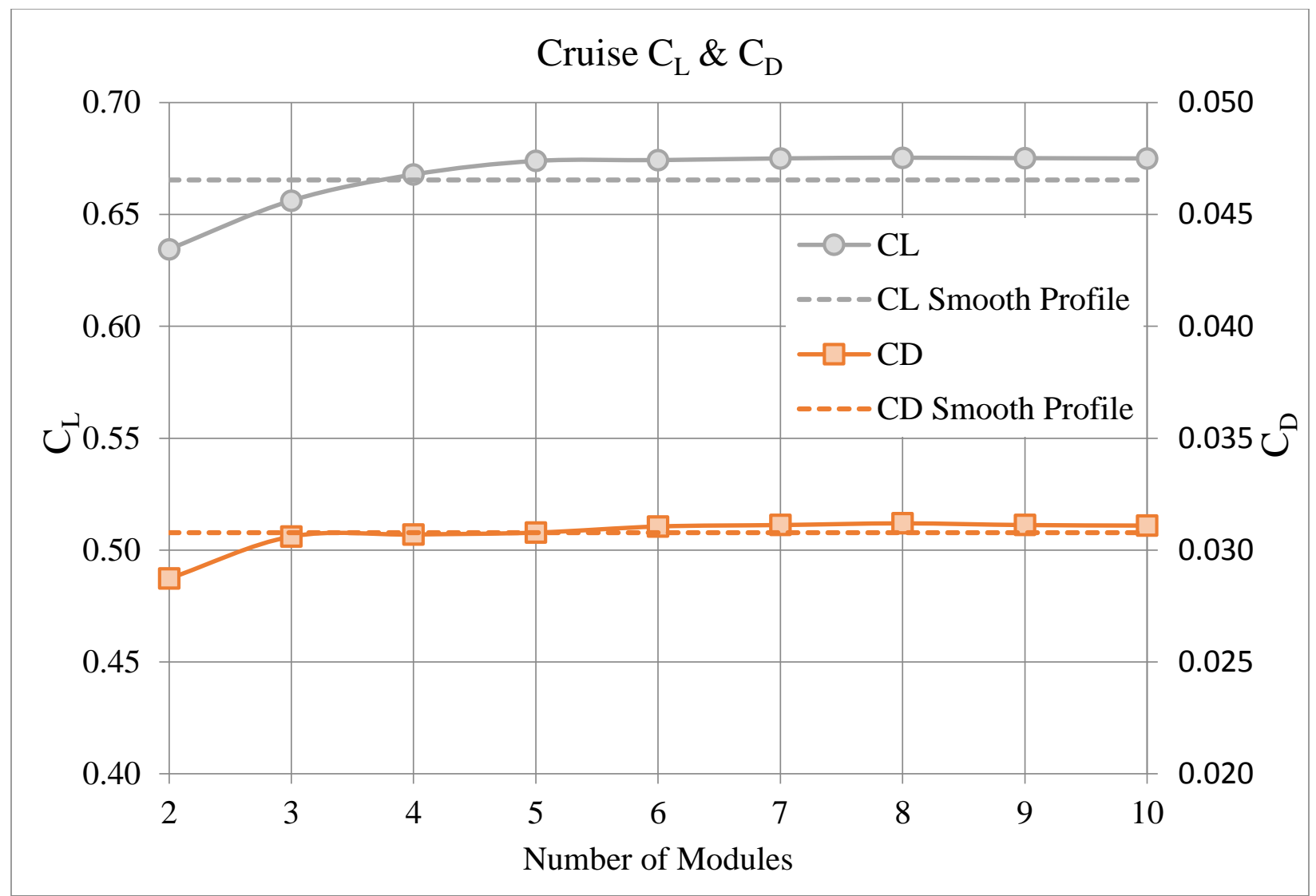

Figure 4.23: Cruise Discretization $\mathrm{C}_{\mathrm{L}} \& \mathrm{C}_{\mathrm{D}}$

Notice figure 4.23 displays the same trend as shown in figure 4.22 where $C_{L}$ and $C_{D}$ change until it plateaus to a close value of the smooth profile case. Although there is seemingly a large difference in $\mathrm{C}_{\mathrm{L}}$ of modules 6 to 10 and the smooth profile, rest assured the actual difference is about $1.4 \%$ which could likely be from the error of slightly different grid formulation between a modular and smooth wing. This shows that even though the number of modules did not show any significant impact on the performance index in Figure 4.20 , the $\mathrm{C}_{\mathrm{L}} \&$ $\mathrm{C}_{\mathrm{D}}$ values separately show to converge toward the smooth profile's performance.

Finally figure 4.24 shows a similar trend as the previous cruise figure. 


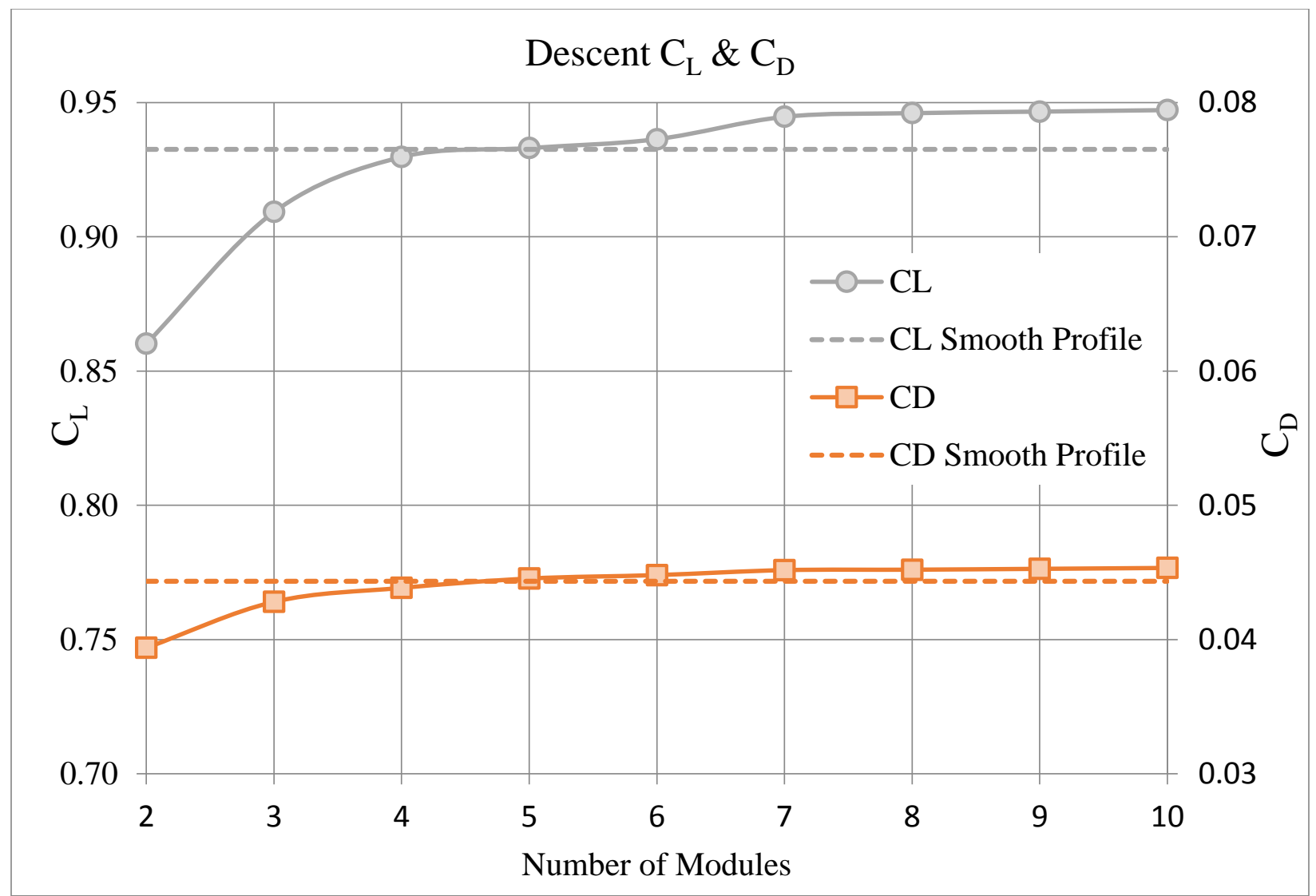

Figure 4.24: Descent Discretization $C_{L} \& C_{D}$

Notice once again that in Figure 4.21 from the previous section, there was no significant impact on the performance index with changing number of modules. Figure 4.24 shows that $\mathrm{C}_{\mathrm{L}}$ $\& \mathrm{C}_{\mathrm{D}}$ both converge to close to that of the smooth profile case. There seems to be an increase in $\mathrm{C}_{\mathrm{L}}$ at 7 modules and higher but the actual difference is only about $1.3 \%$.

When comparing the results of the modular wing to that of the smooth profile, the results of the higher module wings are close to the smooth profile's performance. It should be noted that due to the linear segments of the module wing there would always be a difference in performance when compared to a smooth wing where the surface has a curved shape. The linear segments results in averaged parameters for a given module. Although the effect is more severe at lower number of modules, which was why the 2 module cases has the largest difference from the smooth profile's performance, the effects are still present in even a 10 module wing. 


\subsubsection{Aerodynamics at Joint}

An interesting aspect of the CFX results is the aerodynamic in locations around the joints. An example below is shown for the coefficient of pressure, $\mathrm{C}_{\mathrm{P}}$, across the surface of a climb configuration with 3 modules.

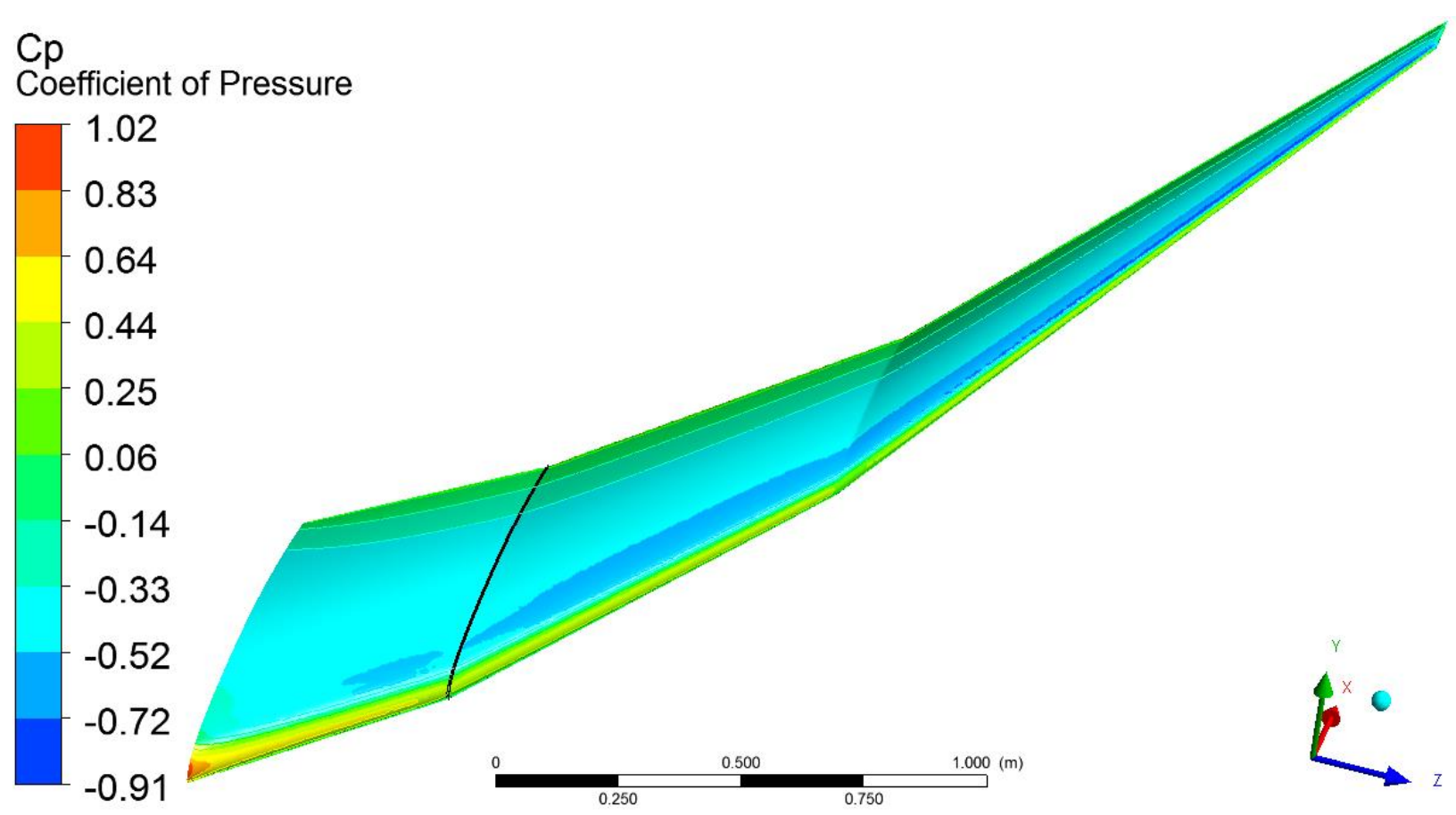

Figure 4.25: Climb 3 Modules $\mathrm{C}_{\mathrm{P}}$

Figure 4.25 shows the upper wing surface (see coordinate frame in figure 4.25). The root is located at the left of the figure. Clearly around the first joint from the root (black line) of the wing there is an increase in surface pressure on the upper surface. The change in value is small which could be why the performance index figures in section 4.4.1 did not reveal dramatic decreases in performance when increasing the number of modules.

The wall shear stress along the surface of the upper surface also shows a change around the joint as shown in the following figure. 


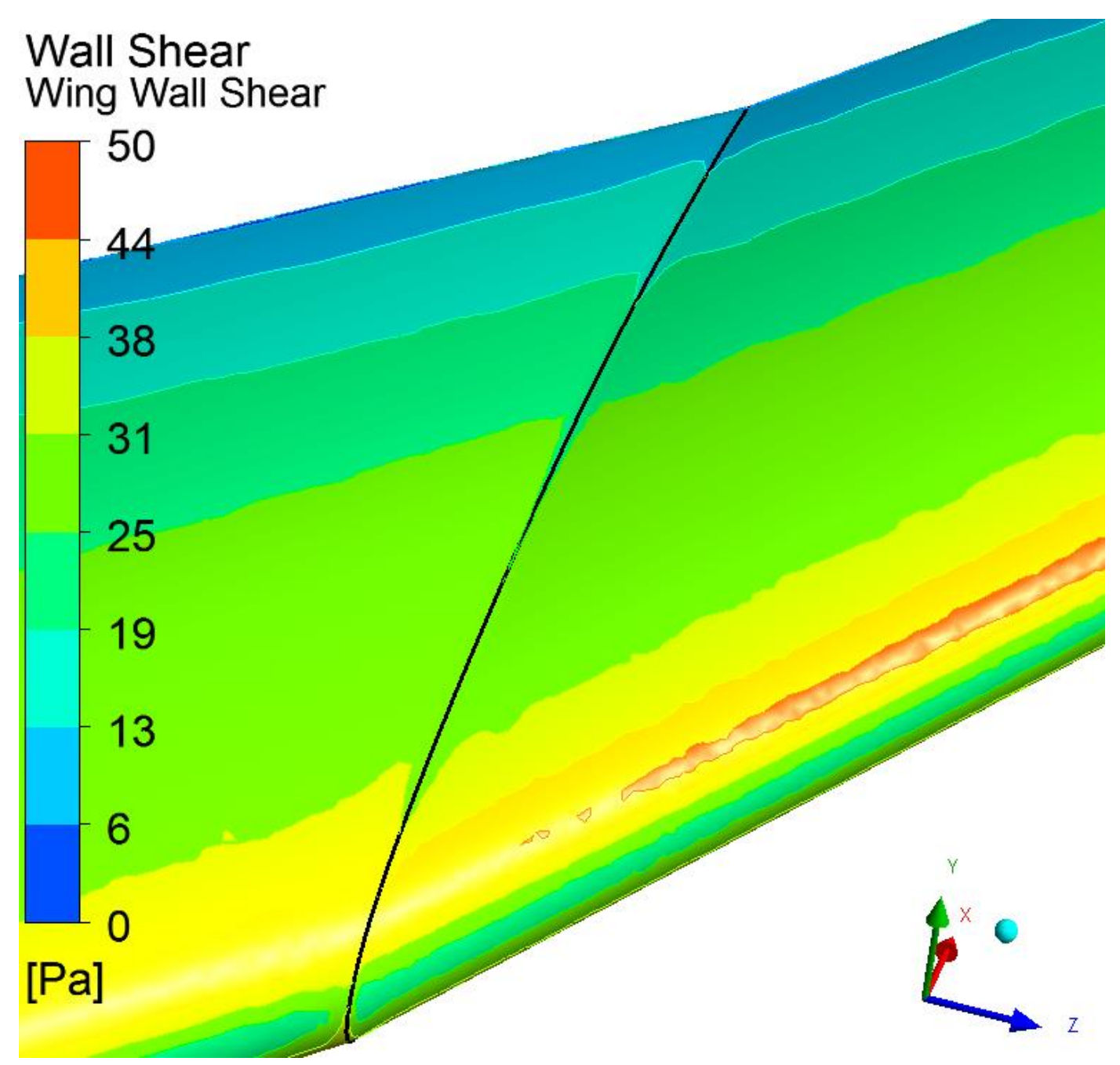

Figure 4.26: Climb 3 Module Wall Shear Stress

Figure 4.26 shows the same joint (black line) shown in figure 4.25 on the upper surface of the 3 module wing geometry for the climb condition. Notice the wall shear stress decreases at the joint location. Although again the value is small the trend is clearly visible especially near the leading edge of the wing (lower part of figure 4.26).

Since these effects do not seemingly have significant impact on the analysis run in this chapter the joint effect on aerodynamics won't be looked into more detail in this section. These effects do however appear to be present and a final conclusion can be made in section 5.4.1. 


\section{Wing Consolidation}

The discretized wings for the three flight regimes were chosen in the previous chapter, the final step is to combine these wing configurations into a single wing. As the true benefit of a morphing wing is the ability to change its configuration depending on the flight objective, the consolidations step is therefore a vital step so that discretized wings can be used in a real world application. This chapter goes through the process of combining the three discretized wings. As outlined by figure 5.1 .

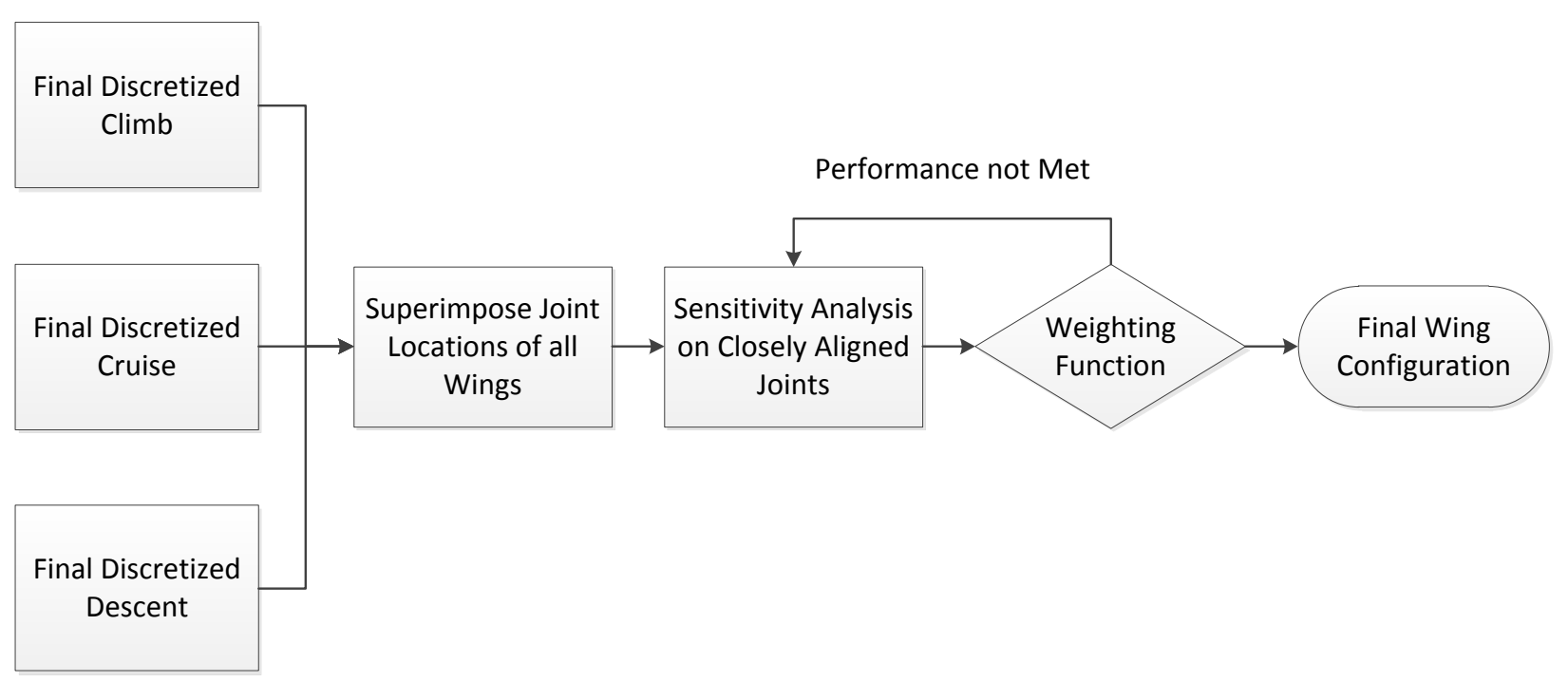

Figure 5.1: Wing Consolidation Method 


\subsection{Discretized Profile Summation}

The consolidation step involves combining joint locations of the three separate wings, chosen in the previous step, into a single wing geometry. As noted from the previous section, the climb flight regime requires 3 modules and both cruise and descent wings only required 2 without sacrificing the aerodynamic performance. Figure 5.2 displays the nodal spacing from the chosen wings in section 4.4 then the combined nodal spacing where the three discretized wings are superimposed.

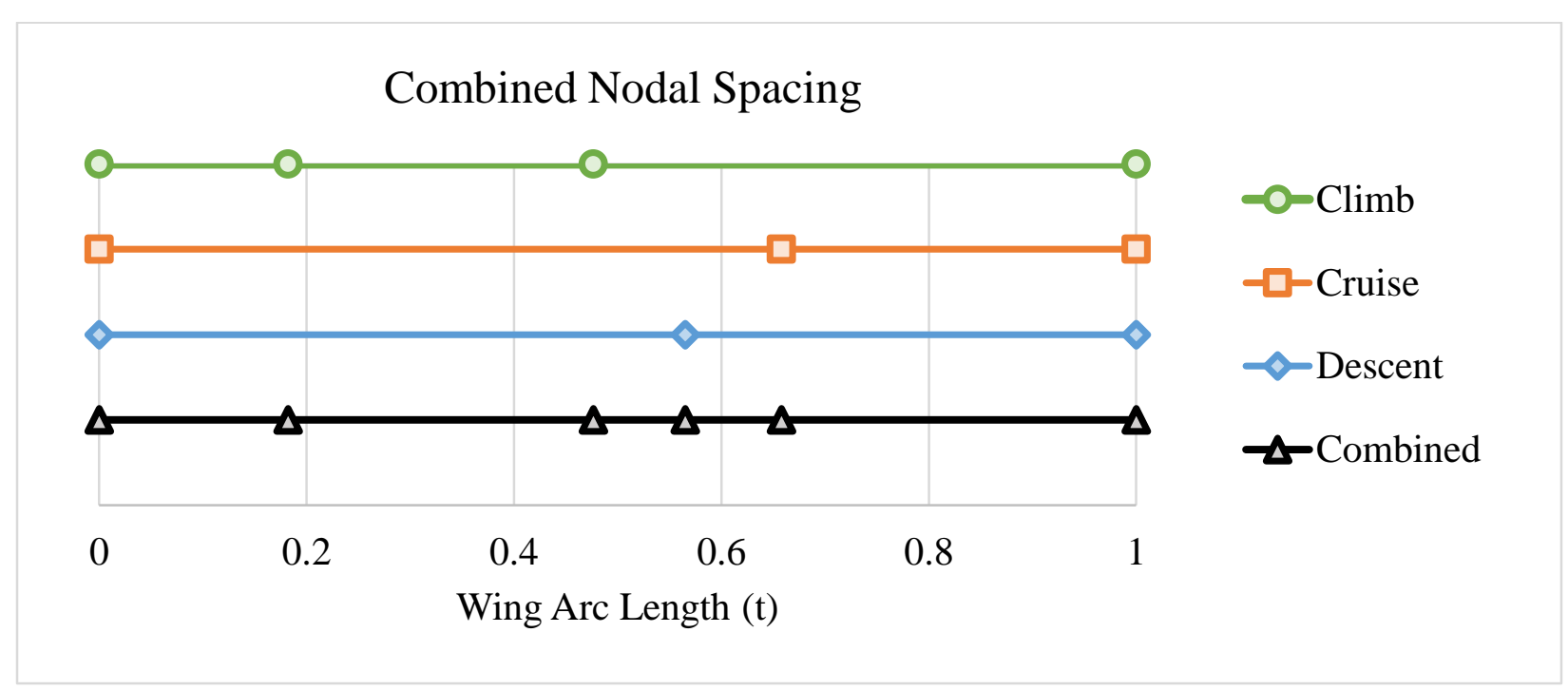

Figure 5.2: Combined Nodal Spacing

The specific nodal spacing for each wing in the previous section is, $T_{\text {climb }}=\{0,0.18$, $0.48,1\}, \mathrm{T}_{\text {cruise }}=\{0,0.66,1\}, \mathrm{T}_{\text {descent }}=\{0,0.57,1\}$. Thus the combined nodal spacing is, $\mathrm{T}_{\text {combined }}=\{0,0.18,0.48,0.57,0.66,1\}$.

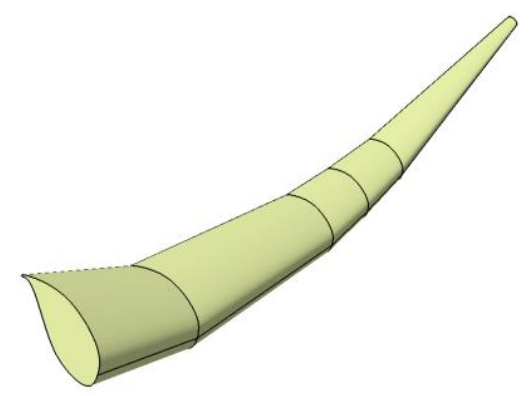

Climb

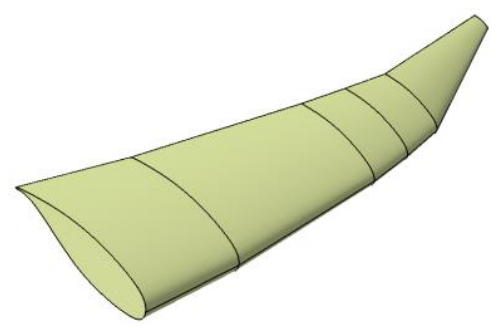

Cruise

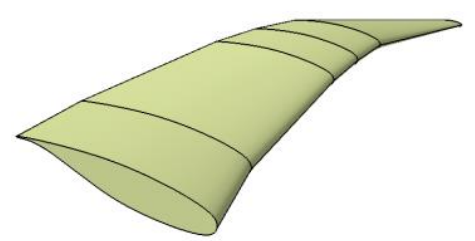

Descent

Figure 5.3: Combined Wing 
Since three joints are closely aligned, further wing consolidation can occur as per figure 5.1. The combined wing, in the above figure, must be evaluated for each flight regime to determine their respective performance index. This represents the beginning of the sensitivity analysis where the joint locations' (within the $0.48 \leq \mathrm{t} \leq 0.66$ region) effect on performance is measured.

\subsection{Sensitivity Analysis}

This section discusses the sensitivity analysis completed to define the optimal joint location for each flight regime. A weight factor is applied in the following section so that the optimal joint location can be determine based on the overall performance for all flight regimes.

An analysis was performed on the combined wing shown in figure 5.3 at each flight regime. Additional to this the three closely aligned joints, $\mathrm{T}=\{0.48,0.57,0.66\}$, are replaced by one joint which would make up the trial runs for the sensitivity. Therefore the three trials that contain the original joints are then:

$$
\begin{aligned}
& T_{\text {trial } 0.48}=\{0,0.18,0.48,1\} \\
& T_{\text {trial } 0.57}=\{0,0.18,0.57,1\} \\
& T_{\text {trial } 0.66}=\{0,0.18,0.66,1\}
\end{aligned}
$$

The above nodal spacing will also be noted as 'original joints' in the figures within this section for the sake of simplicity. Additional to this two extra trials with nodal spacing between those of the three trials above. These are,

$$
\begin{aligned}
& T_{\text {trial0.52 }}=\{0,0.18,0.52 .1\} \\
& T_{\text {trialo.61 }}=\{0,0.18,0.61,1\}
\end{aligned}
$$

The two additional trials is part of the sensitivity analysis so that the performance of configurations between the original joints is considered.

Figure 5.4 shows the climb performance index results for the 3 Module case from section 4.4.1, $\mathrm{T}_{\text {combined, }}$ and the 5 trials from above. 


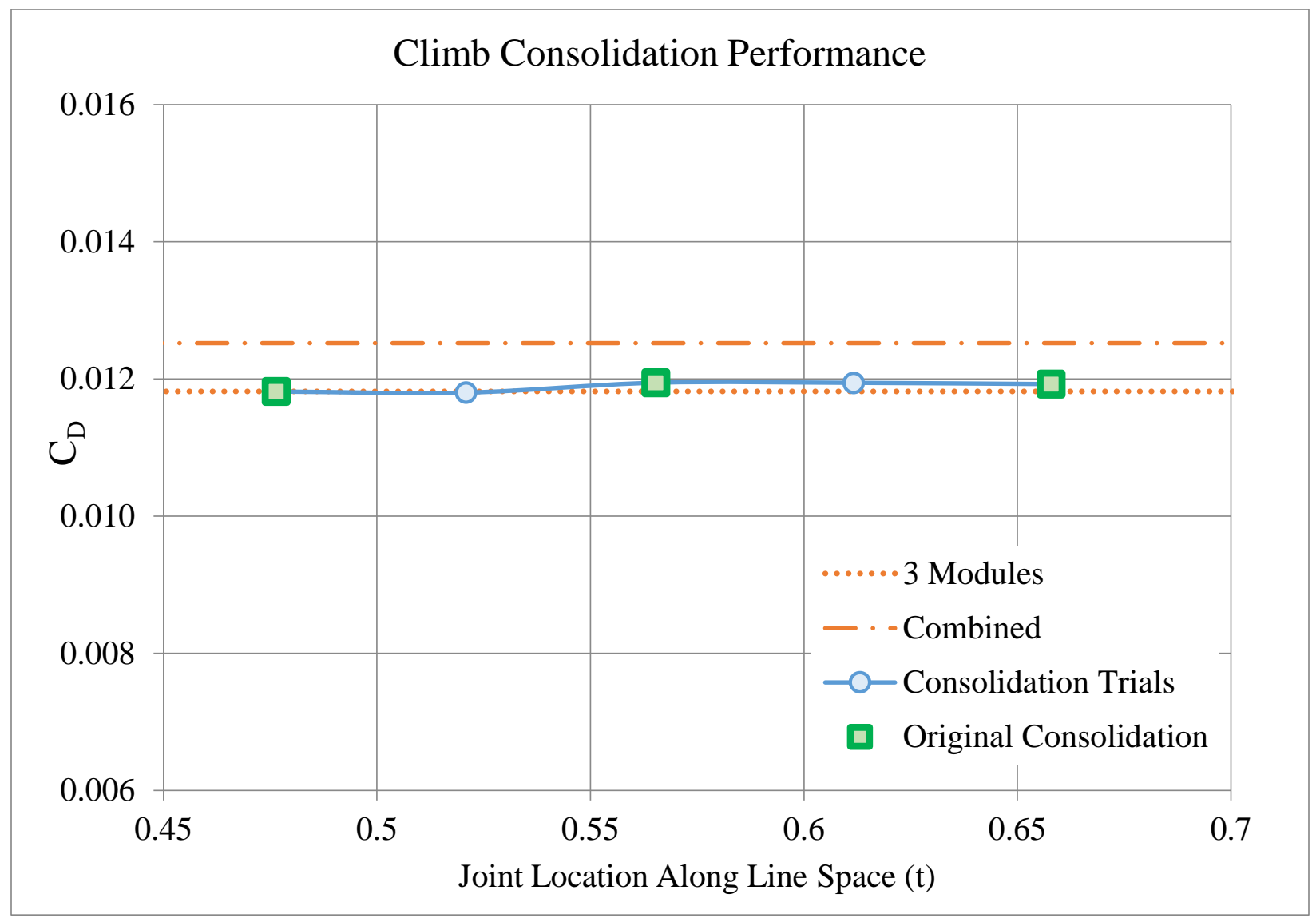

Figure 5.4: Climb Sensitivity Analysis

It is important to note that the 3 Module case from section 4.4.1 is identical to $\mathrm{T}_{\text {trial0.48. }}$. Notice the trials have very similar results in terms of $C_{D}$ but all have performed better than the combined case as the lower $\mathrm{C}_{\mathrm{D}}$ equals to better performance at climb (see section 3.2).

Figure 5.5 shows the results for the same wings' nodal spacing from the above figure but all placed in the cruise configuration. 


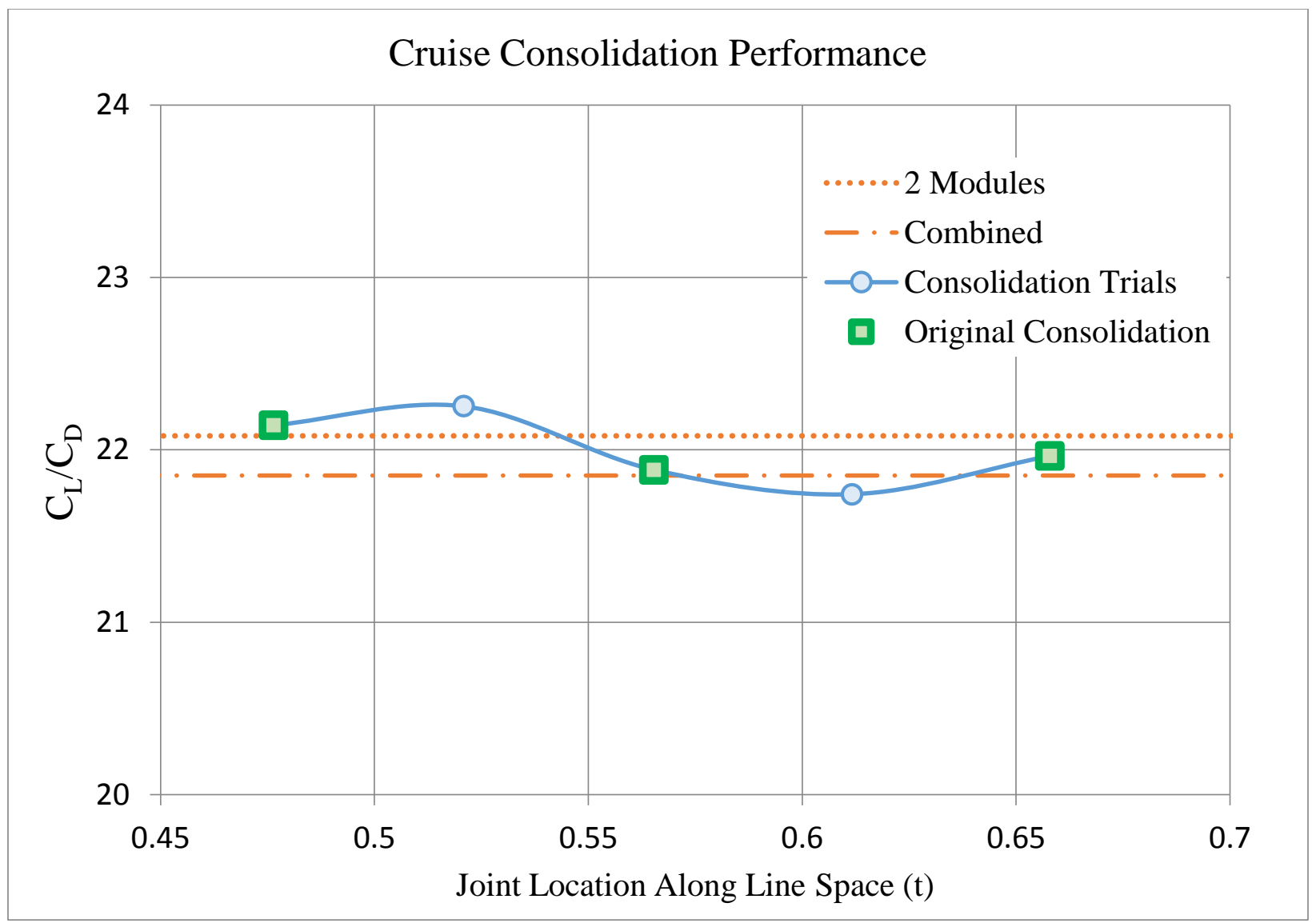

Figure 5.5: Cruise Sensitivity Analysis

In this case the higher $C_{L} / C_{D}$ ratio is better. Although the performance index does not

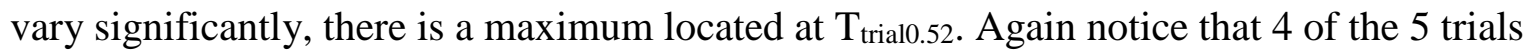
performed better than the combined case.

Finally the descent case is shown in figure 5.6 with the 2 module wing from section 4.4.3, combined, and trials. 


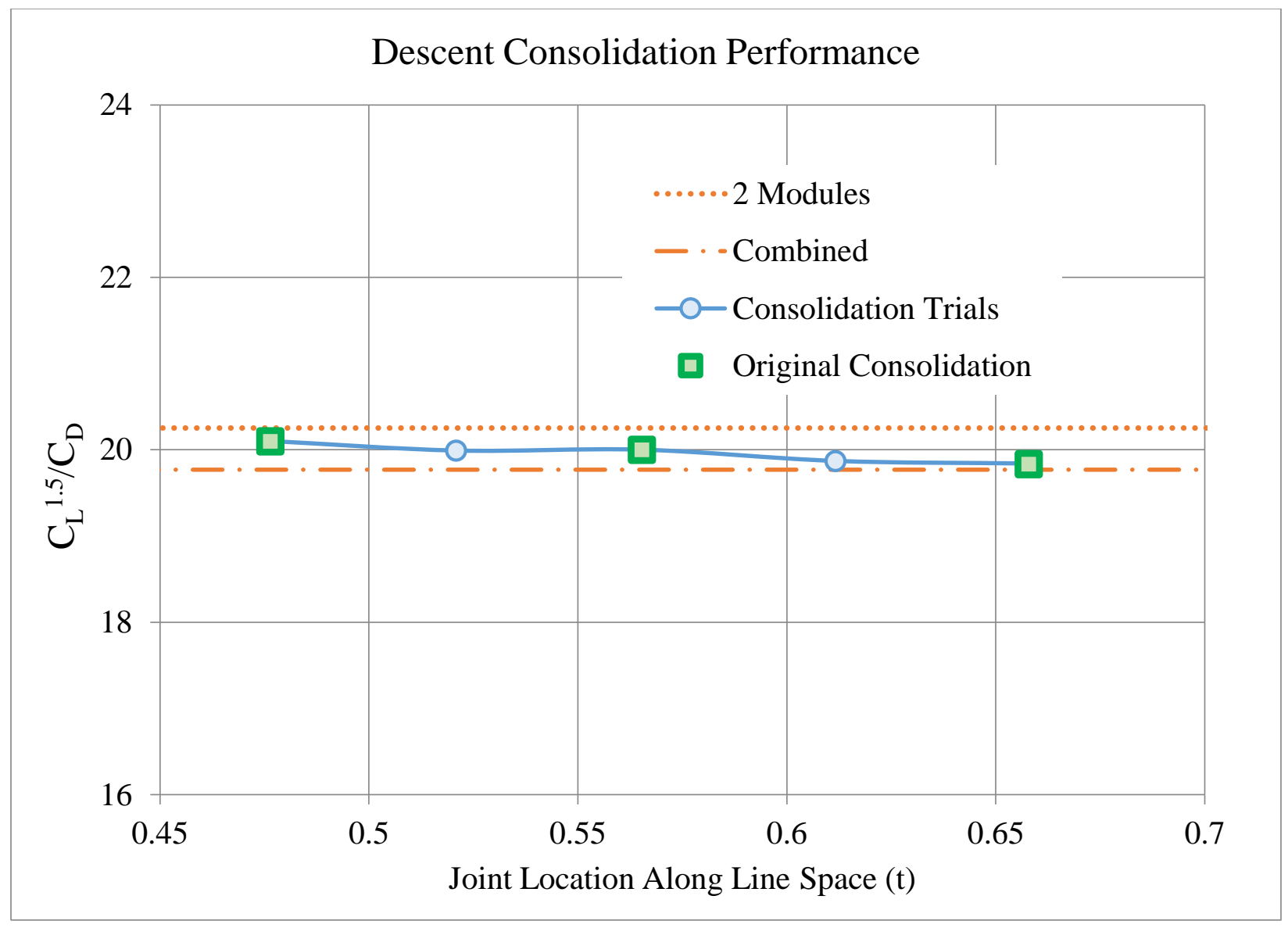

Figure 5.6: Descent Sensitivity Analysis

The higher $\mathrm{C}_{\mathrm{L}}{ }^{1.5} / \mathrm{C}_{\mathrm{D}}$ represents performance during the descent flight regime. Although none of the values performed as well as the original 2 module case from section 4.4.3, the difference is only by a small amount.

From figures 5.4 through 5.6, the common findings is that the cruise case showed the most change with the various consolidation trials. Although the climb and descent cases did not have as strong of an impact, the weighting factor can still be applied now that the sensitivity analysis is completed. Also the joining lines between each trial in the above figures represent only an approximation of what the performance values would be if additional trials were placed with a joint located along that curve. These curves do not represent exact values since with every new trial it should be updated to fit the most recent data. As will be discussed in the next section, 
if a joint location was chosen along this curve, a new trial must be analyzed to confirm the accuracy.

\subsection{Weighting Function}

Using the sensitivity analysis from the previous section, a weight function can be added where priority is set to specific flight regimes. Although all flight regimes have an impact on the overall efficiency of an aircraft, their relative importance may differ depending on the actual flight mission. A long range commercial aircraft would have more emphasis on the cruise performance while short range aircraft may increase the priority of the climb segments.

The first step is to take the performance values of the 5 trials done in the sensitivity analysis and record them into an array. For climb the performance index will be flipped meaning it'll be maximizing $1 / \mathrm{C}_{\mathrm{D}}$ so then all performance index will be that of a maximize function.

$$
\begin{gathered}
\text { Climb Performance Index }=\{84.64,84.75,83.74,83.75,83.88\} \\
\text { Cruise Performance Index }=\{22.14,22.25,21.88,21.74,21.96\} \\
\text { Descent Performance Index }=\{20.10,19.99,20.00,19.87,19.84\}
\end{gathered}
$$

The above performance index arrays are in order from left to right of the trials, so they're the variable joint location is in respect to

$$
\text { Sensitivity Joint Location }=\{0.48,0.52,0.57,0.61,0.66\}
$$

To continue the trials' performance must be put into relative terms of the combined wing case placed in the following array format

$$
r=\left(\begin{array}{llll}
r_{1} & r_{2} & \ldots & r_{i}
\end{array}\right)
$$

Where $r_{i}$ represents the relative performance of the trial vs the combined wing which is shown by equation (5.2).

$$
r_{i}=\frac{\text { Trial }_{i} \text { Performance Index }}{\text { Combined Wing Performance Index }}
$$

Where $\mathrm{i}$ in this case represents the number of trials. For this example the flight regimes climb, cruise, and descent, will be denoted by 'a', 'b', and 'c', respectively. 
The combined case had a performance index of 79.87, 21.85, 19.77, for climb, cruise, and descent, respectively. Using equations (5.1) and (5.2) we have the following

$$
\begin{aligned}
& \text { Climb Relative Performance }=a=\{1.0597,1.0611,1.0485,1.0486,1.0502\} \\
& \text { Cruise Relative Performance }=b=\{1.0133,1.0184,1.0015,0.9950,1.0052\} \\
& \text { Descent Relative Performance }=c=\{1.0166,1.0114,1.0116,1.0053,1.0033\}
\end{aligned}
$$

With the relative performance arrays completed the weighting function can be applied. For this example we can consider the cruise to be the most important, followed by climb, then descent. Thus the chosen values for this example is $25 \%$ for climb, $70 \%$ for cruise, and $5 \%$ for descent. The actual weight can vary but it's important to divide it into a percentage so the sum adds to $100 \%$ for simplicity purposes. The weight function (wf) will be in the following format,

$$
w f=\left(\begin{array}{llll}
w f_{1} & w f_{2} & \ldots w
\end{array}\right)
$$

The component $\mathrm{wf}_{\mathrm{i}}$ is calculated by,

$$
w f_{i}=a_{w e i g h t} \% a_{i}+b_{w e i g h t} \% * b_{i}+c_{w e i g h t} \% * c_{i}
$$

Where $\mathrm{a}_{\text {weight }} \%$ represents the weights discussed earlier for the flight regimes. wf is the resultant weighting function array that contains the summation of the weighting function for each trial

Applying the weighting values mentioned earlier we have,

$$
\begin{gathered}
w f_{i}=(25 \%) a_{i}+(70 \%) * b_{i}+(5 \%) * c_{i} \\
w f=\{1.025,1.029,1.014,1.009,1.016\}
\end{gathered}
$$

Based on the above wf array the $2^{\text {nd }}$ trial point is the suitable joint location. Thus the final wing nodal spacing is,

$$
T_{\text {FinalWing }}=T_{\text {trail } 0.52}=\{0,0.18,0.52,1\}
$$

It is also important to note that if the sensitivity analysis involved more trials then the array portion can be replaced with an approximation function. The idea is to define the trial 
performances as a function similar to the profile smoothing process. Applied the weight function would be done in a similar fashion and would take the form of,

$$
w f_{i}=a_{\text {weight } \%} * f_{a}(i)+b_{\text {weight } \%} * f_{b}(i) * b_{i}+c_{\text {weight } \%} * f_{c}(i)
$$

Where $f_{a}, f_{b}, f_{c}$, represents the approximation function for climb, cruise, and descent performance indices vs the joint location. In this particular case the line joining the trials in figures 5.4 through 5.6 .

Although the sensitivity analysis showed little change in performance indices as the joint location moved, this procedure was shown to successfully determine the consolidated joint location based on performance values for multiple flight regimes.

\subsection{Confirm Final Wing}

The final step is to confirm the difference in performance of the final wing is close to that of the reference wing found in chapter 3 . The following table shows the $C_{L}, C_{D}, \&$ performance index comparison.

Table 5.1: Reference vs Final Wing Performance

\begin{tabular}{|c|l|l|l|}
\hline & \multicolumn{1}{|c|}{ Climb } & \multicolumn{1}{c|}{ Cruise } & \multicolumn{1}{c|}{ Descent } \\
\hline CFX Smooth & CL: 0.198 & CL: 0.665 & CL: 0.932 \\
& CD: 0.0112 & CD: 0.0308 & CD: 0.0443 \\
& PI: 0.0112 & PI: 21.62 & PI: 20.31 \\
\hline Final Wing & CL: 0.270 & CL: 0626 & CL: 0.94714 \\
& CD: 0.0118 & CD: 0.0281 & CD: 0.0461 \\
& PI: 0.0118 & PI: 22.25 & PI: 19.99 \\
\hline Performance & \multicolumn{1}{|c|}{$-5.4 \%$} & $+2.9 \%$ & $-1.6 \%$ \\
Index Change & & & \\
\hline
\end{tabular}

As can be seen the difference in performance index is low. Both climb and descent cases saw a reduction in performance by $5.4 \%$ and $1.6 \%$, respectively. Cruise actually increased in performance but only by a slight margin of $2.9 \%$. The comparison shows that the reference wings were successfully discretized and consolidated into a single wing of only 3 modules without significantly sacrificing the overall aerodynamic performance. 
It is also important to review the progress of the wing shapes from the smooth profile, to discretized configuration, then combined joint locations, and finally the final wing configuration. The following table shows these wings for the three flight regimes.

Table 5.2: Initial to Final Wing Comparison

\begin{tabular}{|c|ccc|}
\hline $\begin{array}{c}\text { Flight } \\
\text { Regime }\end{array}$ & Smooth Profile & Discretized & Combined \\
\hline Climb & &
\end{tabular}

The important note to make of table 5.2 is that even with the number of steps done the final wings still resemble closely to that of the smooth initial profile.

\subsubsection{Aerodynamics at Joint Discussion}

Before concluding this research a point should be made about the effect of the joints on the aerodynamic performance. Discovered by Finistauri [4], as more modules were added an adverse effect was observed where the increase in joints caused the performance index to reduce. It was suspected at the time that the aerodynamics about the joint causes lower pressure so that the wing's overall aerodynamic performance was impacted. As mentioned in section 4.5.1, there was a slight visible change in $C_{P}$ across the surface of the climb case at the joint closes to the root. The following figure shows the same trend (red line) for the combined wing at the climb configuration. 


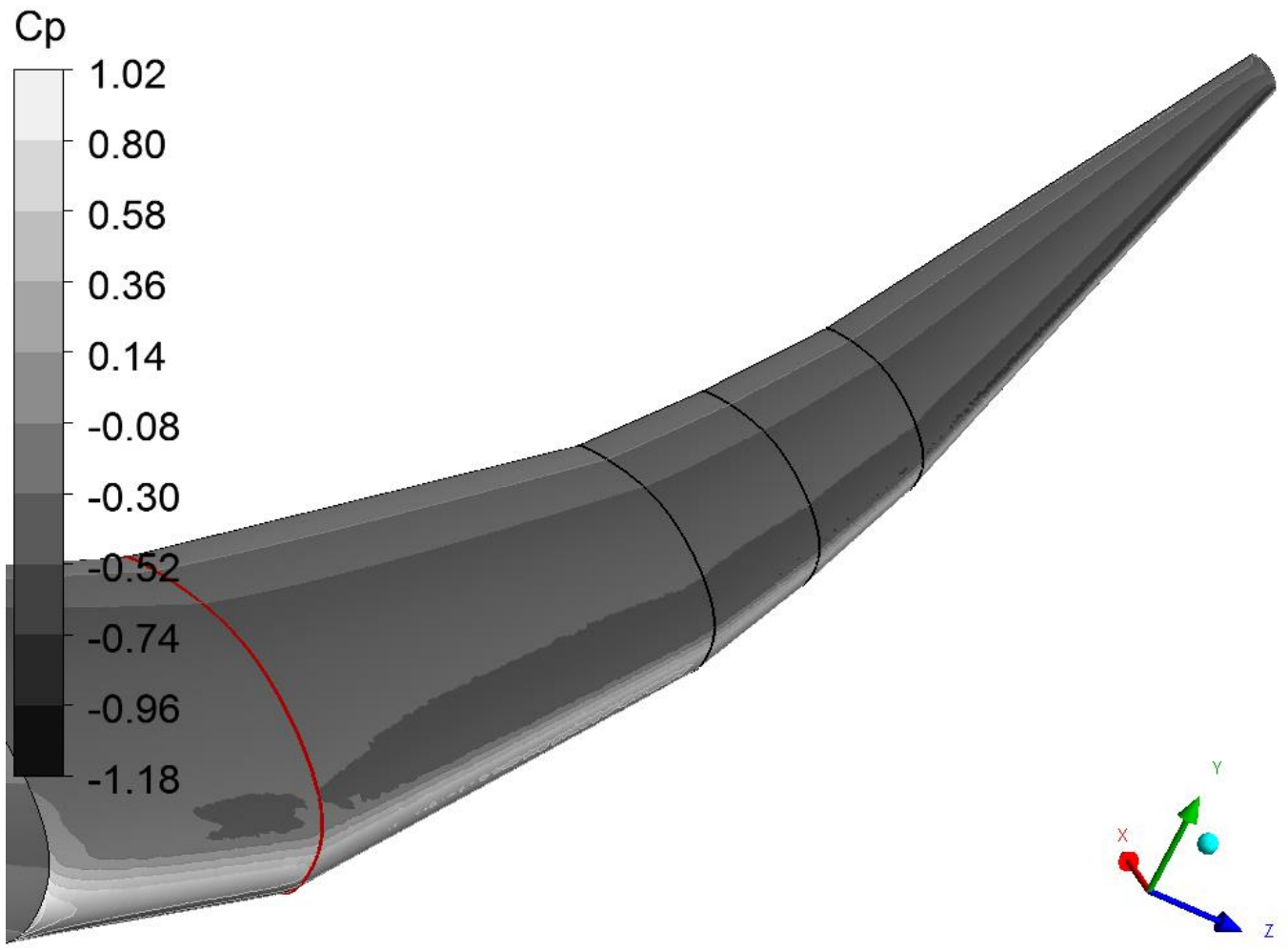

Figure 5.7: Climb Combined $\mathrm{C}_{\mathrm{P}}$

It is interesting to note however that the cruise and descent configurations did not show a similar kind of trend as seen in the following figures, 5.8 and 5.9. 


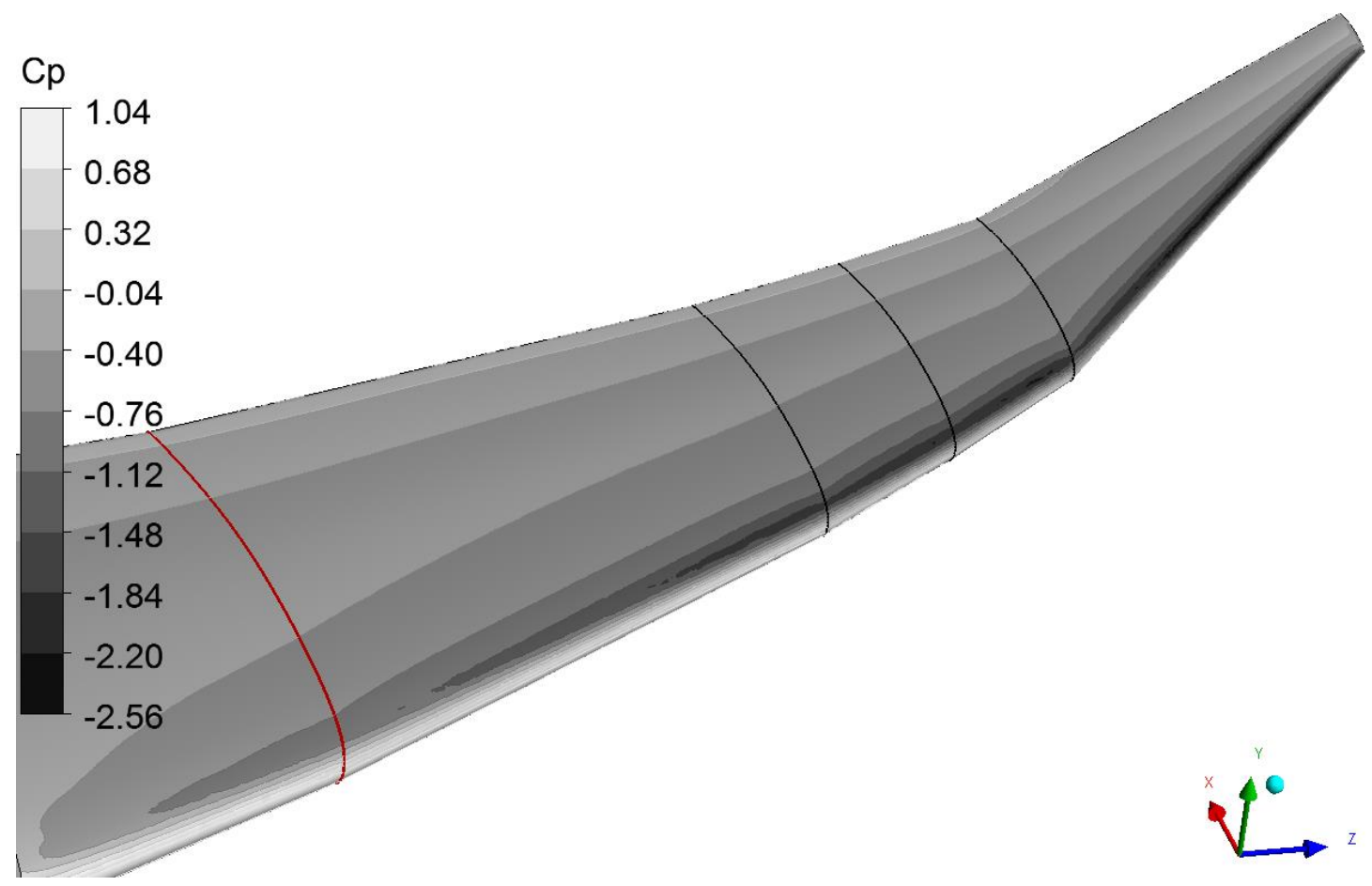

Figure 5.8: Cruise Combined $C_{P}$

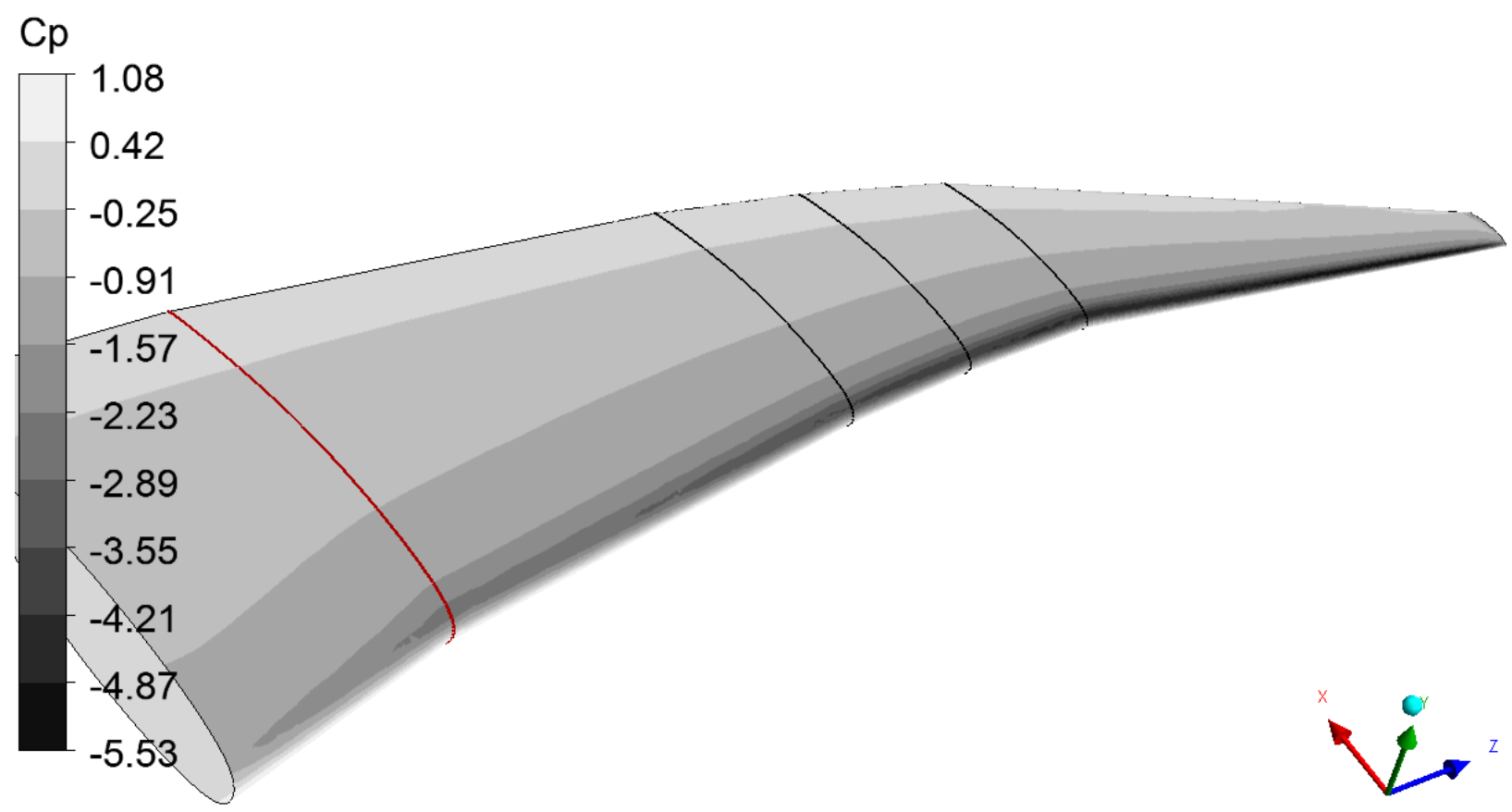

Figure 5.9: Descent Combined $C_{P}$ 
Note the above figures were intentionally placed in black and white so that a clear difference between each band can be seen. The actual $\mathrm{CP}$ values are not important to view the trend in figure 5.7, while figures $5.8 \& 5.9$ have no such trend.

To look more closely at the climb configuration, the following figure shows the wall shear at the climb joint and once again a change can be seen especially close to the leading edge (lower part of figure).

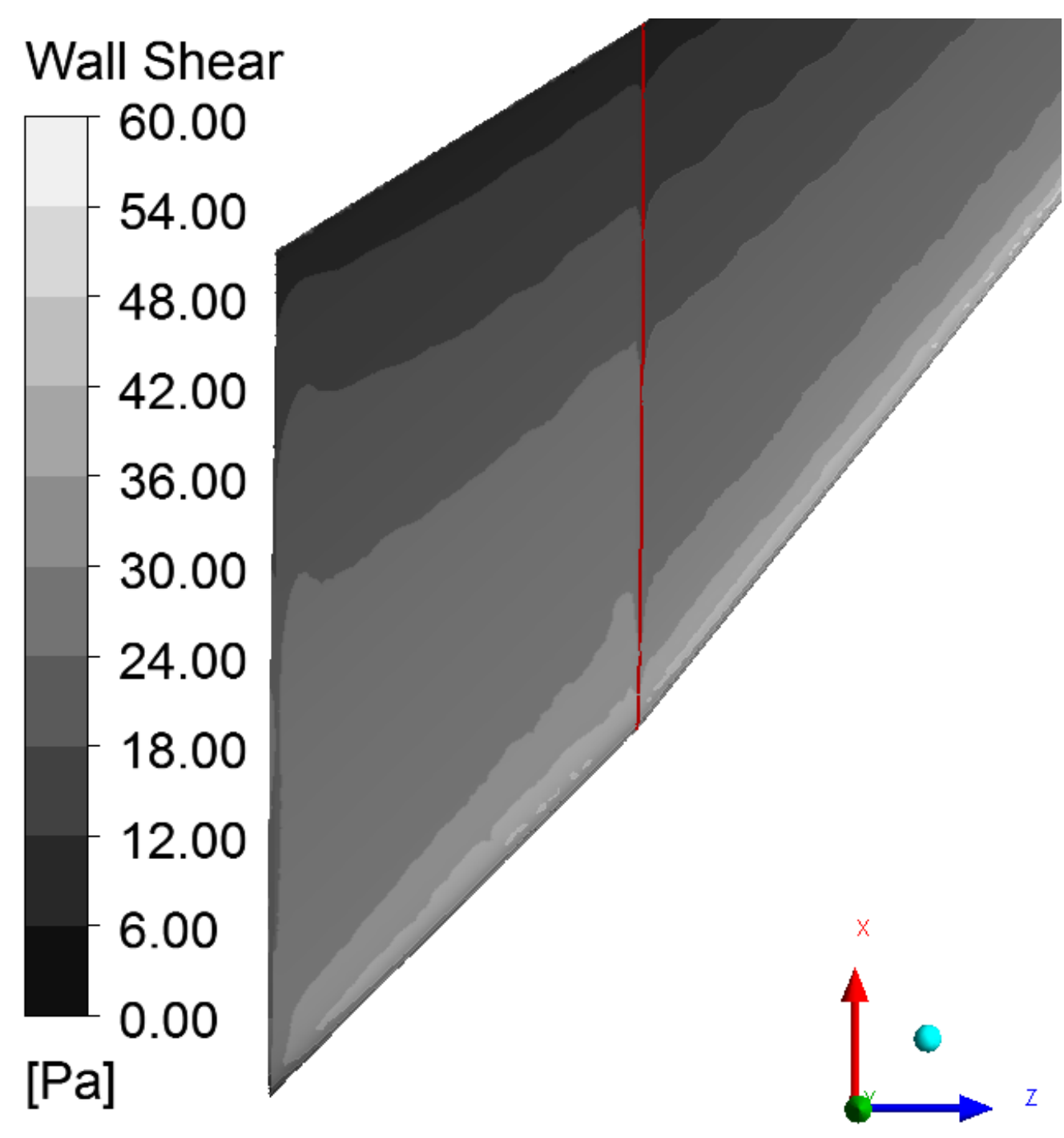

Figure 5.10: Climb Joint Wall Shear

The following plot was created to show the $\mathrm{C}_{\mathrm{P}}$ along the chord at the joint (red line) and adjacent points $5 \%$ of the span toward the left and right. 


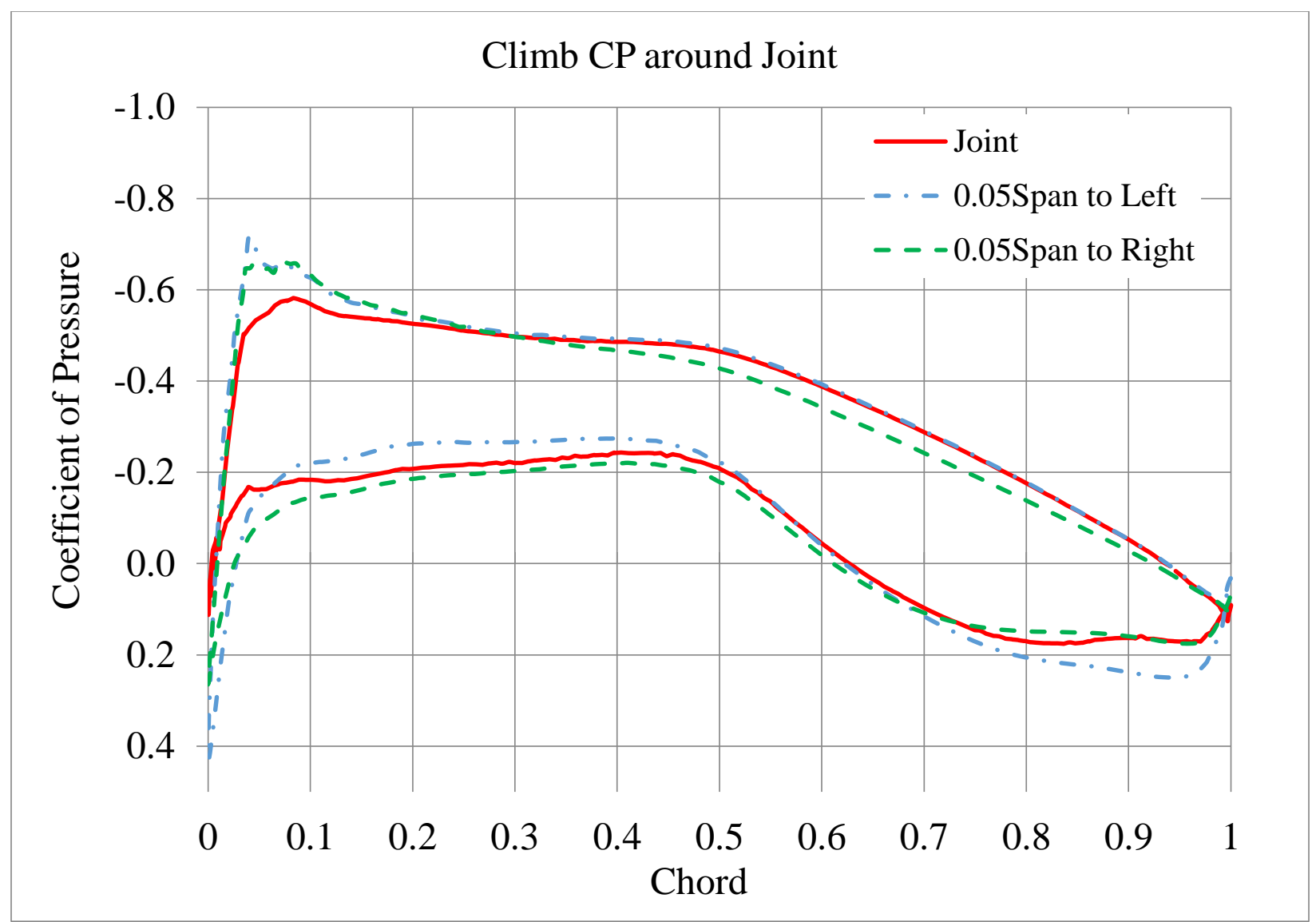

Figure 5.11: Climb Wing, $\mathrm{C}_{\mathrm{P}}$ around Joint

As can be seen a clear difference is present near the leading edge but this seems to even out by the time the flow reaches past the $1 / 4$ chord point. It should be noted that the chord profile to the right of the joint (closer to the wing tip) is expected to have less suction pressure on the upper surface as there is a $9^{\circ}$ dihedral from the left to the right of the joint.

The following figure is in the same format as figure 5.11 where the joint and adjacent chord cross sections along the span are plotted but for wall shear instead of $\mathrm{C}_{\mathrm{P}}$. 


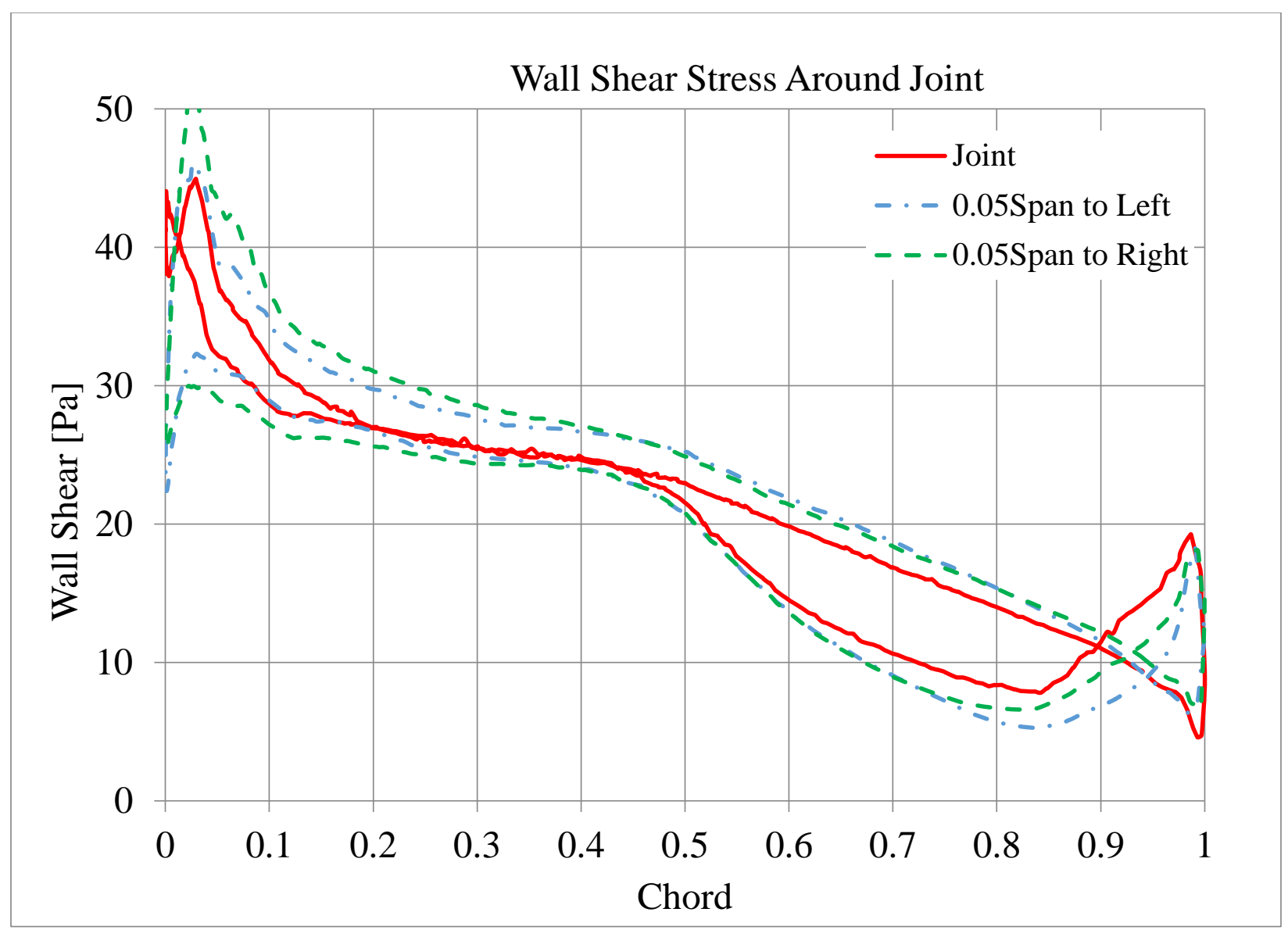

Figure 5.12: Climb Wing, Wall Shear Around Joint

The lines in figure 5.12 represent the wall shear of the upper and lower surface along the wing chord at the joint and adjacent cross sections. The upper lines in the figure represent the upper surface while the lower lines represent the lower surface. There is a clear decrease in wall shear along the joint when compared to the adjacent points. To understand why this happens we'd need to look at the general equation for shear stress [26],

$$
\tau=\mu \frac{\partial u}{\partial y}
$$

Where $\tau$ shear stress, $\mu$ is the dynamic viscosity, $\partial u / \partial y$ is the local shear. Since the $C_{P}$ values are not affected except for at the first $25 \%$ of the chord around the joint, this would be because of the boundary layer thickness. The boundary layer near the leading edge of the wing has not had time to fully form yet when compared to further down the chord. The thicker, fully developed, boundary layer could act almost as a buffer between the discontinuous shape change 
of the joint and the air above the boundary layer. Due to the relative $9^{\circ}$ dihedral between the two adjacent modules air from both sides of the joint are being pushed toward the joint itself thus causing higher pressure and lower velocity. This lower flow velocity would cause the boundary layer to be thicker than sections adjacent to the joint thus lowering the shear stress.

The important note is that the only joint (out of the combined wing configurations) which shows noticeable changes in wall shear and $C_{P}$ was the first joint of the climb case. The relative dihedral of the joint closer to the root for the climb case was $9^{\circ}$. All other joints were less than $5^{\circ}$. Also the change in $\mathrm{C}_{P}$ is for such a short section of the wing that it had very little impact on the overall performance. The decrease in wall shear along the joint means there is instability issues as separation may occur closer to the leading edge when compared to adjacent areas of the joint. This did not seem to effect the total performance but future research may need to check this behaviour with higher angles of attack.

Based on the results obtained in this research the aerodynamic around the joint would only be an issue if the relative dihedral was at least $9^{\circ}$ between adjacent modules and multiple closely spaced joints all experience this high amount of change. When considering the early findings Finistauri found [4], the reference wing used in that study resembled a typical aircraft wing with a winglet. This means that most of the wing had a low dihedral distribution then a drastic change occurred closer to the wing tip. The root of the wing tip could then fit the criteria mentioned earlier about multiple closely aligned joints where the majority of the relative dihedral between adjacent modules was at least $9^{\circ}$.

In this particular thesis' methodology the initial wing profiles experience more gradual change as the entire wing was being optimized. This, in combination with the discretization means as the number of modules increases (chances for closely aligned joints), the relative dihedral between joints would then decrease. This could then only be an issue if a reference wings with more abrupt changes were found such as that of a winglet. Although the consolidation technique could experience multiple joints closely together, the purpose of the technique is to combine those joints thus minimizing the issue of adverse aerodynamic effects from multiple joints. 


\section{Conclusions and Future Work}

This thesis presented the design of a modular morphing wing suited for cruise, climb, and descent flight regimes by changing sweep, cant, twist, and span parameters. With the increase in research for morphing aircraft technology, this research successfully establishes a method to consolidate multiple discretized wings into a single wing capable of morphing into multiple flight regimes. The modular morphing wing concept was based on prior research by Finistauri et al [4] which was primarily focused on the morphing mechanisms. This research performs thorough aerodynamic analysis for a modular morphing wing and presents the steps involved for finding wing profiles suited for the three flight regimes.

Wing profiles were found initially using the harmony search algorithm in conjunction with a vortex lattice method. This optimization was done for each flight regime separately. The resultant wing geometries were different in shape due to optimized performance at their respective flight regimes. The climb wing profile had a high sweep and dihedral distribution which allowed for minimizing drag suited for the best rate of climb. The cruise wing profile had a flat shape with little dihedral. There was an increase sweep near the wing tip which allowed for the best lift to drag ratio as needed for improved range performance. Finally the descent configuration had a larger front profile due to high twist distributions which allowed for higher lift at the expense of drag. This was suited for the performance index for minimizing the sink rate. 
The module discretization method was setup using Ansys CFX so that an in depth analysis can be done on modular wings with varying number of modules. The cruise and descent cases were found to have little performance benefit when using more than 2 modules. The climb case did show a benefit in performance when moving from a 2 module wing to 3 , but with increasing number of modules (4 to 10 modules) there was no significant benefit in performance. Choosing a discretized wing with the fewest number of modules helps minimize the complexity of the system. An increase in modules for a morphing wing results in added weight, energy to change shape, and increased complexity which could lead to a decrease in reliability. Although the quantity of these effects was not within the scope of this thesis, using the fewest number of modules possible is still an important objective.

A new consolidation method was introduced so that separate discretized wings can be combined into a single final wing. Since each discretized wing has separate joint locations, these needed to be combined then further consolidated so that closely aligned joints were replaced by a single joint. This step involved performing a sensitivity analysis and weighting function so that priority is given for each flight regime. This particular research gave the priority to cruise, followed by climb, then descent. It was outlined that this can vary depending on the flight mission. The resultant wing had nodal spacing of $T_{\text {FinalWing }}=\{0,0.18,0.52,1\}$. Thus the final wing was reduced from 5 modules when the three discretized wings were combined, to a 3 module wing. The performance values of the final wing compared to the initial smooth wings found was a decrease of only $5 \%$ and $2 \%$ for climb and descent, respectively. The cruise case was found to have an increase in performance by $3 \%$. The final wings were then considered a success as the original wing profiles were converted into a modular wing without significant reduction in performance.

\subsection{Contribution}

Although the wing profiles were not originally investigated to find the best possible shape, a method that allows the user more control over the wing profile optimization routine was presented so that future work can be improved with minimal parameter noise. This method is universal as it can be applied to new performance objectives. 
The discretization method was applied for climb, cruise, and descent. Although a similar study was done previously using a vortex lattice method for a winglet design [dis3], this research incorporates a finite element computational fluid solver so that drag can be more accurately analyzed based on viscous effects. This then effectively lead to the following contributions of the consolidation technique as well as the aerodynamics around the module joint locations.

A new consolidation technique was presented so that the discretization method can be applied toward a real world application where multiple flight regimes are considered. The importance of this step was the weighting function as it should not be assumed that all flight regimes have equal importance. This adds room for further efficiency especially when used with a common performance parameter such as fuel consumption. This would then help quantify the importance of each configuration and help determine which flight regimes benefit the most from a modular morphing wing.

The aerodynamics around the joint location presented interesting results as the boundary layer from the adjacent modules intersect. It was previously found that these had an adverse effect on aerodynamic performance when more modules was added by Finistauri [4]. These areas were then considered but it was found that they did not have a significant impact for the wing profiles examined within this thesis. Although there was a loss in pressure along the joint due to the thinner boundary layer near the leading edge, it was determined that adjacent modules observed in this research with a relative dihedral of 9 degrees or less did not have noticeable performance loss when considering the entire wing. The particular wing that Finistauri used resembled that of on a typical aircraft where a winglet is present. This mean the majority of the span had a low dihedral and then sharp change in dihedral occurred near the wing tip to create a winglet. This leads to multiple joints located close together at the winglet root where each of them have a relatively higher dihedral angle from one another. This type of geometry did not occur within this thesis where a dramatic change was concentrated in a particular location along the wing span, instead the optimization routine generated profiles with gradual change. This would explain the lack of adverse effects more joints had in this research when compared to that of Finistauri [4]. It was determined that care for the potential adverse effects of the joints must be 
considered if multiple joints are closely aligned and each has at least a 9 degree relative dihedral. Otherwise no observable effect is expected.

\subsection{Future Work}

Some areas of future research have already been discussed previously within this chapter. One of those being to integrate more steps in the optimization procedure where gradual steps are made to increase the parameter constraints and number of modules [see section 3.6]. This helps provide the user with support when deciding where more modules may be needed as well as deciding upon suitable parameter constraints.

The consolidation weighting function was based on a common performance value. In this research the common performance value was to compare the consolidation trials to the combined wing case. Future research could look at other performance indicators such as fuel consumption. Fuel consumption would then outline a direct benefit each wing configuration has so that a better weighting function can be determined. This would require an analysis specific to a particular airplane so then thrust, weight, and required energy to perform morphing would need to be considered.

Future analysis for consolidation would be better suited finding a common performance variable in relation to the performance indices. A good example would be the fuel consumption. This way the initial steps taken for relative performance can be ignored as the fuel consumption is a common parameter that all flight regimes can relate directly to.

Another area for future research was discussed in section 5.4.1, is to research the effects of the joint discontinuity in surface at the joints. This particular study should use a reference wing with a sharp change in geometry such as that of a winglet case. This would also help determine the type of wing skin necessary surrounding the module joints in a more finalized design. Checking the separation point along the joint for higher angles of attack would also be important to consider.

Other areas of course would involve a more in depth analysis to the flight regimes involved. Although the objective was met to find wing configurations suitable for climb, cruise, 
and descent flight regimes. Broadening the focus on slightly off designs by adjusting the boundary conditions would help establish a more robust design. One example of this would be vary the angle of attack.

Another area, which was out of the scope of this research, is to consider the aircraft stability for each wing configuration. Each wing's effect on stability would greatly affect the design robustness in real world applications. 


\section{References}

[1] Thill, C., Etches, J., Bond, I., Potter, K., and Weaver, P., “Morphing Skins,” The Aeronautical Journal, Vol. 112, No. 3, 2008, pp. 117-138.

[2] T. A. Weisshaar. Morphing Aircraft Systems: Historical Perspective and Future Challenges. Journal of Aircraft, Vol.50, No.2, March-April 2013.

[3] K. Smith, J. Butt, M. R. von Spakovsky, A Study of the Benefits of Using Morphing Wing Technology in Fighter Aircraft Systems. 39th AIAA Thermophysics Conference, Miami, Florida, 25 - 28 June 2007.

[4] A. D. Finistauri. "Conceptual Design of a Modular Morphing Wing," Ryerson University, Department of Aerospace Engineering, Doctor of Philosophy Thesis, Toronto, Ontario, 2012

[5] Karlene Petitt, Flight to Success: Monday Motivation. February 2012 http://4.bp.blogspot.com/-11IHRIGc1o/Ty2m0N9M3bI/AAAAAAAAF0A/7E2LpHKm8PY/s400/Aircraft\%2BAxis.jpg, Retrieved: July 2014

[6] A. K. Jha, and J. N. Kudva, "Morphing Aircraft Concepts, Classifications, and Challenges," Proceedings of SPIE, Vol. 5388, pp. 213-224, 2004 
[7] S. Vasista, L. Tong, K. C. Wong, Realization of Morphing Wings: A

Multidisciplinary Challenge. Journal of Aircraft, Vol. 49, No. 1, January - February 2012.

[8] J. S. Bae, T. M. Seigler, D. J. Inman. Aerodynamic and Aeroelastic

Considerations of a Variable Span Morphing Wing. 45th AIAA/ASME/ASCE/AHS/ASC

Structures, Structural Dynamics \& Materials Conference, 2004-1726, Palm Springs,

California, 19 - 22 April 2004.

[9] Lert, P., “The fs-29: A Telescoping Wing Sailplane,” Soaring, pp. 21-27, January 1976

[10] Perry, R. L., "Variable Sweep: A Case Study of Multiple Re-Innovation,” AIAA 3rd Annual Meeting, AIAA Paper 1966-0983, 1966.

[11] R. W. Kress. Variable Sweep Wing Design. AIAA Paper 1983-1051, Bethpage, New York, 1983.

[12] M. Abdulrahim and R. Lind. Control and simulation of a multi-role morphing micro air vehicle. In AIAA Guidance, Navigation, and Control Conference and Exhibit, San Francisco, California, 12 - 18 August 2005.

[13] M. Abdulrahim, R. Lind. Flight Testing and Response Characteristics of a Variable Gull-Wing Morphing Aircraft. AIAA Guidance, Navigation, and Control Conference and Exhibit, 2004-5113, Providence, Rhode Island, 16 - 19 August 2004.

[14] S. P. Joshi, A. K. Jha, J. E. Rodrian, R. A. Alphenaar, and K. Szema, "Design of the NextGen Morphing Wing Wind Tunnel Model," AIAA SDM Conference, 2007-1731, Honolulu, Hawaii, April 2007.

[15] J. Bowman, B. Sanders, B. Cannon, J. Kudva, S. Joshi, and T. Weisshaar. Development of Next Generation Morphing Aircraft Structures. 48th AIAA/ASME/ASCE/AHS/ASC Structures, Structural Dynamics, and Material Conference, 2007-1730, Honolulu, Hawaii, 23 - 26 April 2007. 
[16] J. Morley. "Shape Optimization and Modular Discretization for the Development of a Morphing Wingtip," University of Toronto, Department of Mechanical and Industrial Engineering, Master of Applied Science Thesis, Toronto, Ontario, 2012

[17] A. R. Rodriguez. Morphing aircraft technology survey. 45th AIAA Aerospace Sciences Meeting and Exhibit, 2007-1258, Reno, Nevada, 8-11 January 2007.

[18] J. Bowman, M. B. Sanders, T. Weisshaar. Evaluating the Impact of Morphing Technologies on Aircraft Performance. 43rd AIAA/ASME/ASCE/AHS/ASC Structures, Structural Dynamics, and Materials Conference, 2002-1631, Denver, Colorado, 22 - 25 April 2002.

A. D. Finistauri, F. Xi, P. Walsh, Discretization Method for the Development of a Modular Morphing Wing. Journal of Aircraft, Vol.49, No.1, January - February 2012.

[20] S. P. Joshi, Z. Tidwell, W. A. Crossley, and S. Ramakrishnan. Comparison of morphing wing strategies based upon aircraft performance impacts. $45^{\text {th }}$ AIAA/ASME/ASCE/AHS/ASC Structures, Structural Dynamics \& Materials Conference, 2004-1722, Palm Springs, California, 19 - 22 April 2004.

[21] Z. W. Geem and H. Hwangbo, "Application of Harmony Search to MultiObjective Optimization for Satellite Heat Pipe Design," in Proceedings of 2006 UK-Korea conference of Science, Technology and Enterpreneurship, 2006.

[22] T. Melin, "A Vortex Vattice MATLAB Impelmentation of Linear Aerodynamic Wing Applications," Royal Institute of Technology (KTH). Stockholm, Sweden, December 2000.

[23] J. Anderson. “Aircraft Performance \& Design”. McGraw-Hill Science/Engineering/Math, 1st edition, December 1998.

[24] P. Paudel. "Module Discretization of a Modular Morphing Wing for Descent Configuration," Ryerson University, Department of Aerospace Engineering, Undergraduate Thesis, Toronto, Ontario, 2013. 
[25] R. Perera, P. Paudel, F. Xi, P. Walsh, “ Module Discretization of A Modular Morphing Wing with Three Flight Regimes," 60th Aeronautics Conference and AGM, Toronto, Canada (Abstract Accepted)

[26] J. A. Schetz. "Boundary Layer Analysis". Virginia Polytechnic Institute and State University, Prentice-Hall, Inc., Upper Saddle River New Jersey, 1993 


\section{Appendix}

Table A.1: Climb Nodal Spacing

\begin{tabular}{|c|c|c|c|c|c|c|c|c|c|c|c|}
\hline $\mathbf{m}$ & \multicolumn{11}{|c|}{$\begin{array}{c}\text { Wing Line Space }(\mathbf{t}) \\
\text { Root to Tip }\end{array}$} \\
\hline 2 & 0 & 0.3097 & 1 & & & & & & & & \\
\hline 3 & 0 & 0.1820 & 0.4764 & 1 & & & & & & & \\
\hline 4 & 0 & 0.1278 & 0.3097 & 0.5823 & 1 & & & & & & \\
\hline 5 & 0 & 0.0980 & 0.2296 & 0.4037 & 0.6562 & 1 & & & & & \\
\hline 6 & 0 & 0.0793 & 0.1820 & 0.3097 & 0.4764 & 0.7108 & 1 & & & & \\
\hline 7 & 0 & 0.0665 & 0.1504 & 0.2513 & 0.3752 & 0.5345 & 0.7525 & 1 & & & \\
\hline 8 & 0 & 0.0572 & 0.1278 & 0.2113 & 0.3097 & 0.4299 & 0.5823 & 0.7850 & 1 & & \\
\hline 9 & 0 & 0.0502 & 0.1110 & 0.1820 & 0.2638 & 0.3599 & 0.4764 & 0.6222 & 0.8107 & 1 & \\
\hline 10 & 0 & 0.0447 & 0.0980 & 0.1597 & 0.2296 & 0.3097 & 0.4037 & 0.5165 & 0.6562 & 0.8314 & 1 \\
\hline
\end{tabular}

Table A.2: Cruise Nodal Spacing

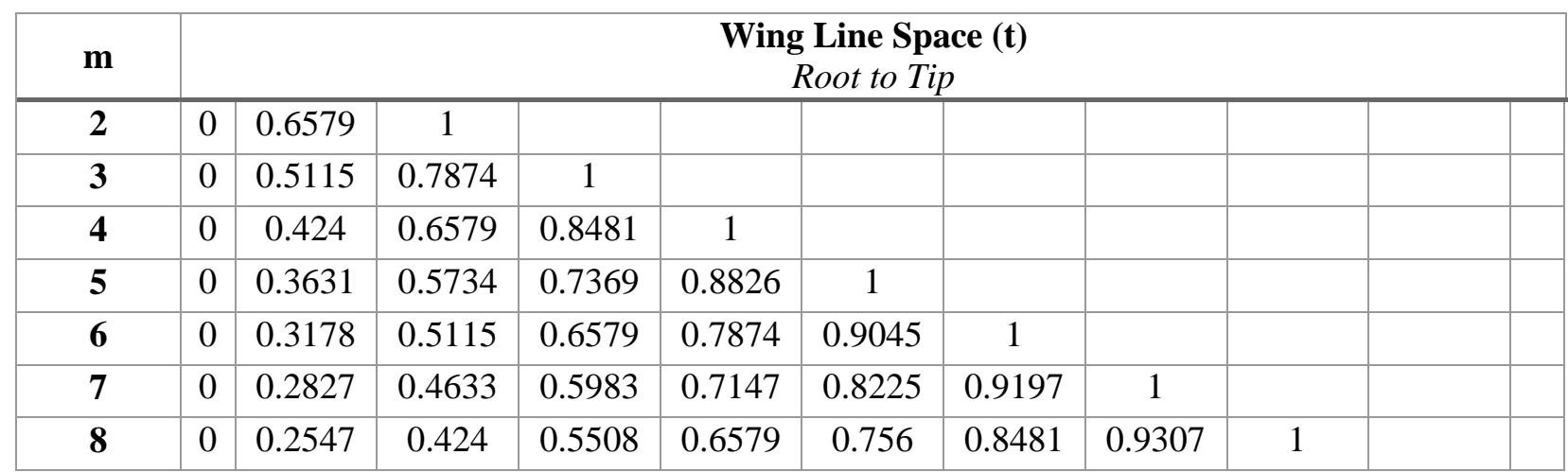




\begin{tabular}{|c|c|c|c|c|c|c|c|c|c|c|c|}
\hline $\mathbf{9}$ & 0 & 0.2318 & 0.3911 & 0.5115 & 0.6119 & 0.7023 & 0.7874 & 0.8675 & 0.939 & 1 & \\
\hline $\mathbf{1 0}$ & 0 & 0.2128 & 0.3631 & 0.4782 & 0.5734 & 0.6579 & 0.7369 & 0.8121 & 0.8826 & 0.9456 & 1 \\
\hline
\end{tabular}

Table A.3: Descent Nodal Spacing

\begin{tabular}{|c|c|c|c|c|c|c|c|c|c|c|c|}
\hline $\mathbf{m}$ & \multicolumn{11}{|c|}{$\begin{array}{c}\text { Wing Line Space (t) } \\
\text { Root to Tip }\end{array}$} \\
\hline 2 & 0 & 0.5653 & 1 & & & & & & & & \\
\hline 3 & 0 & 0.4252 & 0.6796 & 1 & & & & & & & \\
\hline 4 & 0 & 0.2405 & 0.5653 & 0.7477 & 1 & & & & & & \\
\hline 5 & 0 & 0.1592 & 0.4900 & 0.6331 & 0.8087 & 1 & & & & & \\
\hline 6 & 0 & 0.1236 & 0.4252 & 0.5653 & 0.6796 & 0.8960 & 1 & & & & \\
\hline 7 & 0 & 0.1018 & 0.3530 & 0.5131 & 0.6138 & 0.7162 & 0.9249 & 1 & & & \\
\hline 8 & 0 & 0.0868 & 0.2405 & 0.4680 & 0.5653 & 0.6502 & 0.7477 & 0.9396 & 1 & & \\
\hline 9 & 0 & 0.0757 & 0.1885 & 0.4252 & 0.5253 & 0.6032 & 0.6796 & 0.7773 & 0.9492 & 1 & \\
\hline 10 & 0 & 0.0672 & 0.1592 & 0.3798 & 0.4900 & 0.5653 & 0.6331 & 0.7048 & 0.8087 & 0.9560 & 1 \\
\hline
\end{tabular}

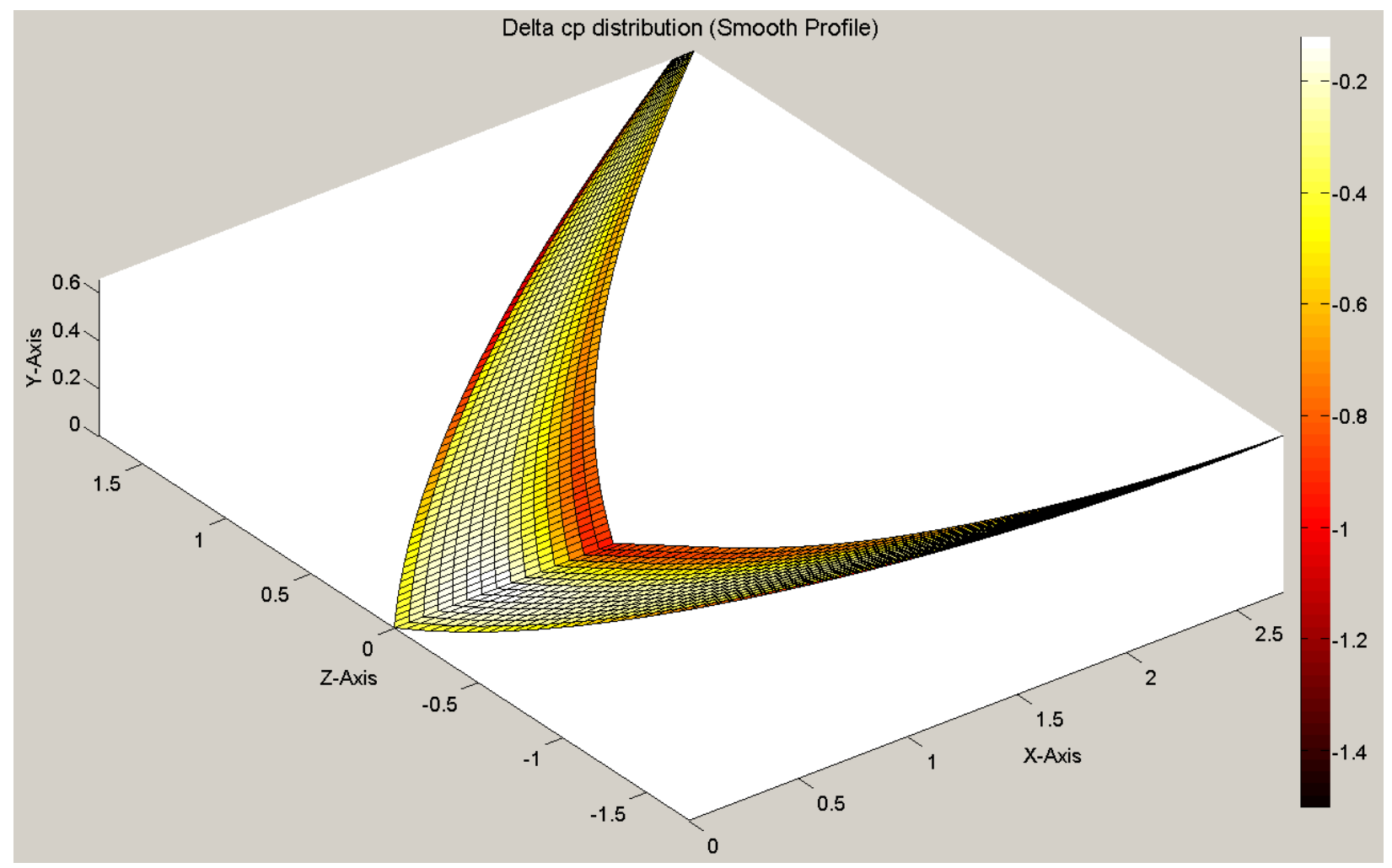

Figure A.1: Climb Smooth Profile Delta CP 


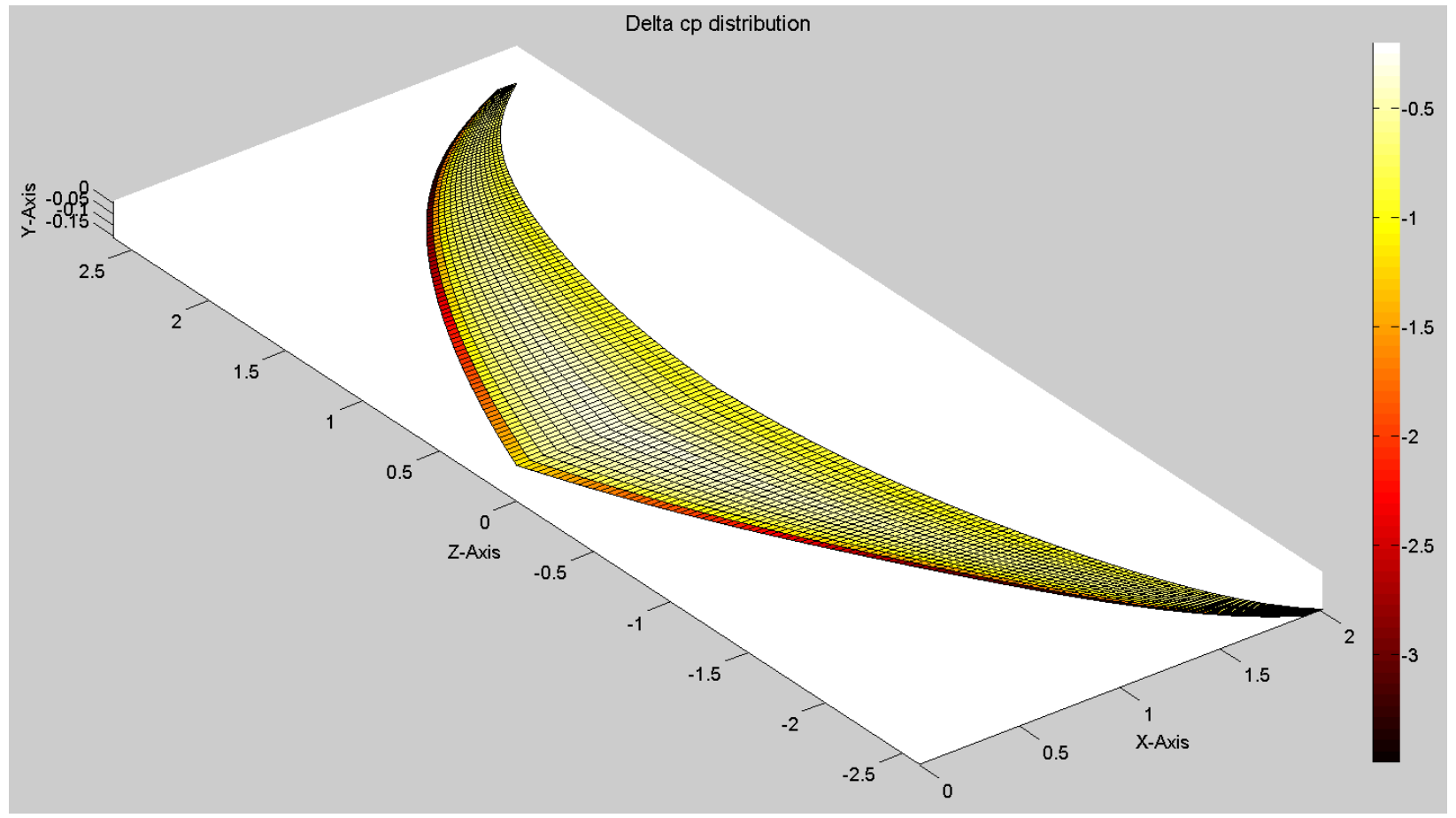

Figure A.2: Cruise Smooth Profile Delta CP

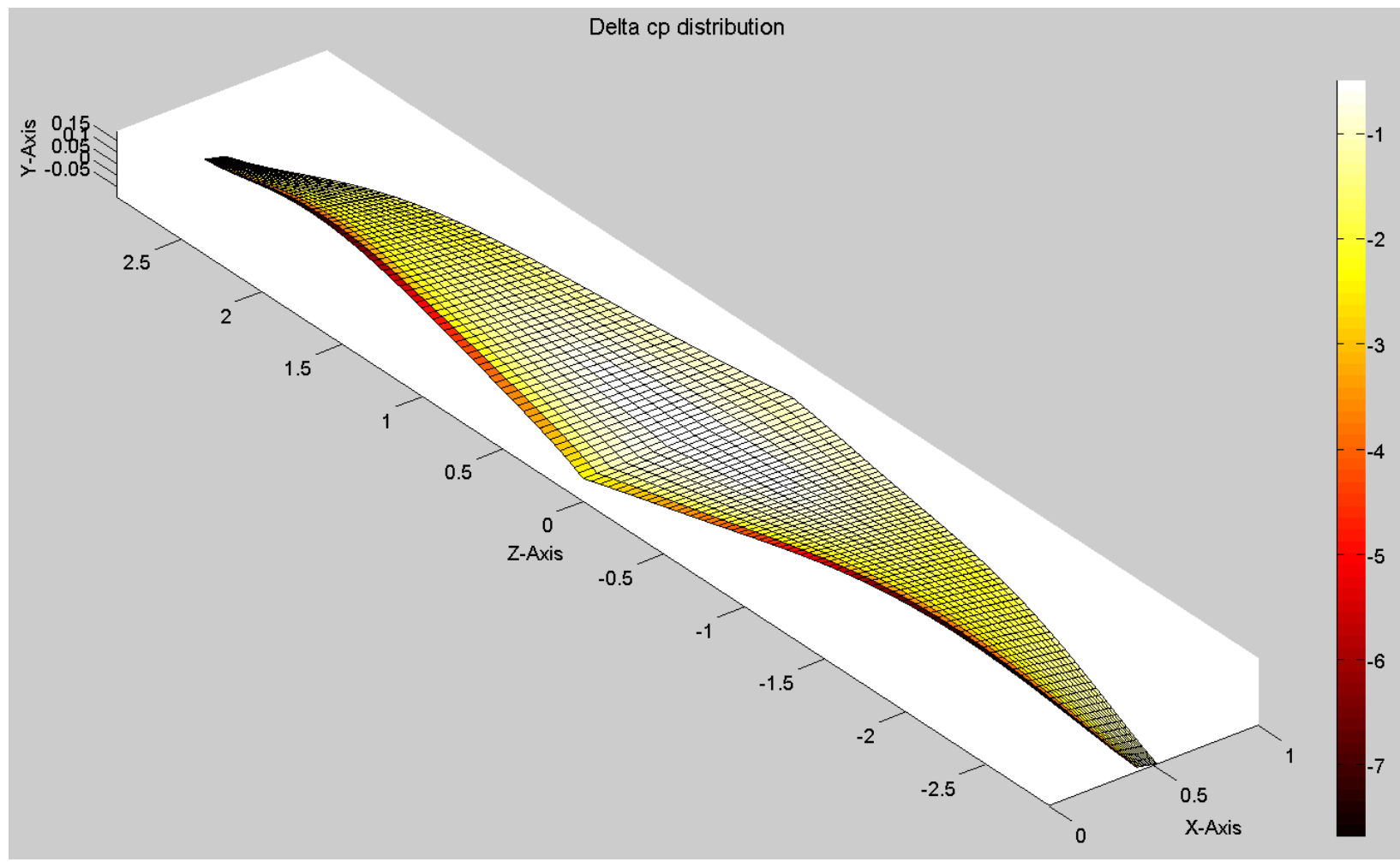

Figure A.3: Descent Smooth Profile Delta CP 
Table A.4: Climb Discretization CFX Performance

\begin{tabular}{|c|c|c|c|}
\hline & \multicolumn{2}{|c|}{ Results } & Performance Index \\
\hline Number of Modules & CL & CD & $1 /$ CD \\
\hline 2 & 0.23289 & 0.01314 & 76.0819 \\
\hline 3 & 0.21322 & 0.01181 & 84.6409 \\
\hline 4 & 0.20734 & 0.01174 & 85.1539 \\
\hline 5 & 0.20515 & 0.01169 & 85.5488 \\
\hline 6 & 0.20297 & 0.01164 & 85.9473 \\
\hline 7 & 0.20198 & 0.01152 & 86.8243 \\
\hline 8 & 0.20075 & 0.01152 & 86.8180 \\
\hline 9 & 0.20135 & 0.01149 & 87.0306 \\
\hline 10 & 0.20195 & 0.01146 & 87.2443 \\
\hline Smooth Profile & 0.1982 & 0.0112 & 89.286 \\
\hline
\end{tabular}

Table A.5: Cruise Discretization CFX Performance

\begin{tabular}{|c|c|c|c|}
\hline & \multicolumn{2}{|c|}{ Results } & Performance Index \\
\hline Number of Modules & CL & CD & CL/CD \\
\hline 2 & 0.63442 & 0.02873 & 22.0814 \\
\hline 3 & 0.65614 & 0.03061 & 21.4341 \\
\hline 4 & 0.66780 & 0.03070 & 21.7524 \\
\hline 5 & 0.67386 & 0.03079 & 21.8885 \\
\hline 6 & 0.67422 & 0.03106 & 21.7070 \\
\hline 7 & 0.67500 & 0.03112 & 21.6874 \\
\hline 8 & 0.67530 & 0.03120 & 21.6442 \\
\hline 9 & 0.67510 & 0.03112 & 21.6907 \\
\hline 10 & 0.67500 & 0.03110 & 21.7035 \\
\hline Smooth Profile & 0.6654 & 0.03078 & 21.616 \\
\hline
\end{tabular}

Table A.6: Descent Discretization CFX Performance

\begin{tabular}{|c|c|c|c|}
\hline & \multicolumn{2}{|c|}{ Results } & Performance Index \\
\hline Number of Modules & CL & CD & 1/CD \\
\hline 2 & 0.86022 & 0.03940 & 20.2518 \\
\hline 3 & 0.90926 & 0.04282 & 20.2498 \\
\hline 4 & 0.92973 & 0.04385 & 20.4419 \\
\hline 5 & 0.93307 & 0.04455 & 20.2308 \\
\hline 6 & 0.93638 & 0.04481 & 20.2220 \\
\hline 7 & 0.94470 & 0.04518 & 20.3249 \\
\hline 8 & 0.94607 & 0.04521 & 20.3546 \\
\hline 9 & 0.94664 & 0.04527 & 20.3437 \\
\hline 10 & 0.94721 & 0.04534 & 20.3328 \\
\hline Smooth Profile & 0.9325 & 0.04435 & 20.3061 \\
\hline
\end{tabular}

